\title{
Matrix factorizations and link homology
}

by

\author{
Mikhail Khovanov (New York) and Lev Rozansky (Chapel Hill, NC)
}

\begin{abstract}
For each positive integer $n$ the HOMFLYPT polynomial of links specializes to a one-variable polynomial that can be recovered from the representation theory of quantum $\operatorname{sl}(n)$. For each such $n$ we build a doubly-graded homology theory of links with this polynomial as the Euler characteristic. The core of our construction utilizes the theory of matrix factorizations, which provide a linear algebra description of maximal Cohen-Macaulay modules on isolated hypersurface singularities.

\section{Contents}

1. Introduction 1

2. The cyclic Koszul complex 11

3. Potentials, isolated singularities, and matrix factorizations $\quad 15$

4. Factorizations as functors 24

5. Homogeneous potentials and graded factorizations 37

6. Planar graphs and factorizations $\quad 40$

7. Tangle diagrams and complexes of factorizations $\quad 68$

8. Invariance under Reidemeister moves $\quad 71$

9. Factorizations and 2-dimensional TQFTs with corners $\quad 80$

10. Projective invariance for cobordisms of tangle $\quad 82$

11. A generalization $\quad 86$

$\begin{array}{lr}\text { References } & 89\end{array}$
\end{abstract}

1. Introduction. The HOMFLYPT polynomial of oriented links in $\mathbb{R}^{3}$ is uniquely determined by the skein relation in Figure 1 and its value on the unknot (see [HOMFLY], [PT]).

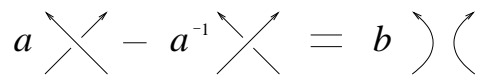

Fig. 1. The HOMFLYPT skein relation

The specialization $a=q^{n}$ and $b=q-q^{-1}$, for integer $n$, produces a onevariable link polynomial which can be interpreted via representation theory

2000 Mathematics Subject Classification: Primary 57M25.

Key words and phrases: matrix factorizations, link homology, HOMFLYPT. 
of the quantum group $U_{q}(\operatorname{sl}(n))$ if $n$ is positive (see $\left.[\mathrm{RT}]\right), U_{q}(\operatorname{sl}(-n))$ if $n$ is negative, and $U_{q}(g l(1 \mid 1))$ if $n=0$ (see $[\mathrm{KS}]$ ). This polynomial is invariant under changing $q$ to $q^{-1}$ simultaneously with passing to the mirror image of the link; therefore, we do not lose any information by restricting to nonnegative $n$. We denote this one-variable polynomial by $P_{n}(L)$, where $L$ is an oriented link; the normalization is

$$
P_{n}(\text { unknot })=[n]:=\frac{q^{n}-q^{-n}}{q-q^{-1}} \quad \text { if } n>0, \quad P_{0}(\text { unknot })=1 .
$$

For $n=0,1,2,3$ there exists a doubly-graded homology theory of links whose Euler characteristic is $P_{n}(L)$. Let us denote this theory by $H_{n}^{\prime}(L)$.

- $P_{0}(L)$ is the Alexander polynomial, and $H_{0}^{\prime}(L)$ was constructed by Peter Ozsváth and Zoltán Szabó [OS], and, independently, by Jacob Rasmussen [Ra]. Their theory exists in greater generality and, in particular, encompasses knots in homology spheres.

- $P_{1}(L)=1$ and $H_{1}^{\prime}(L) \cong \mathbb{Z}$ for any oriented link $L$, with $\mathbb{Z}$ in bidegree $(0,0)$. It will be clear subsequently that this is a natural choice for $H_{1}^{\prime}(L)$.

- $P_{2}(L)$ is the Jones polynomial; $H_{2}^{\prime}(L)$ was defined in [Kh1], and denoted by $\mathcal{H}(L)$ there. $H_{2}^{\prime}$ (unknot) is isomorphic to the integral cohomology ring of the 2-sphere.

- $H_{3}^{\prime}(L)$ was constructed in [Kh3]. $H_{3}^{\prime}$ (unknot) is isomorphic to the integral cohomology ring of $\mathbb{C P}^{2}$.

The goal of the present paper is to construct, for each $n>0$, a doublygraded homology theory $H_{n}(L)$ with Euler characteristic $P_{n}(L)$. The polynomial $P_{n}(L)$ can be computed by breaking up each crossing into a linear combination of diagrams of flat trivalent graphs, as in Figure 2.

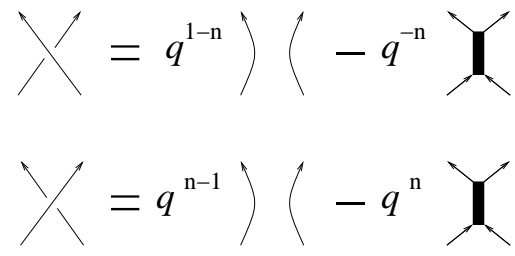

Fig. 2. Reducing to planar graphs

In these planar graphs some edges are oriented so that the neighborhood of each unoriented edge (depicted by a thick line, and referred to from now on as a "wide edge") looks as on the rightmost pictures in Figure 2. Two oriented edges "enter" the wide edge at one vertex and two oriented edges "leave" it at the other. Of course, this arrangement could be used to provide each wide edge with a canonical orientation, but we will not need it. In 
addition, oriented loops are allowed (an oriented loop is a crossingless plane projection of the oriented unknot).

There is a unique way to assign a Laurent polynomial $P_{n}(\Gamma) \in \mathbb{Z}\left[q, q^{-1}\right]$ to each such graph $\Gamma$ so as to satisfy all skein relations in Figure 3.

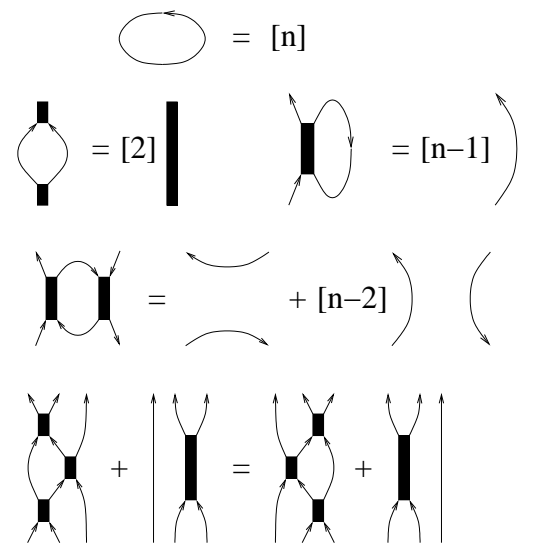

Fig. 3. Graph skein relations, $[i]=\frac{q^{i}-q^{-i}}{q-q^{-1}}$

In the representation theory language, an oriented edge stands for the vector representation $V$ of quantum $s l(n)$, and a wide edge for its (quantum) exterior power $\Lambda^{2} V$. The trivalent vertex is the unique (up to scaling) intertwiner between $V^{\otimes 2}$ and $\Lambda^{2} V$. The polynomial $P_{n}(\Gamma)$ has nonnegative coefficients (see $[\mathrm{MOY}]$ ).
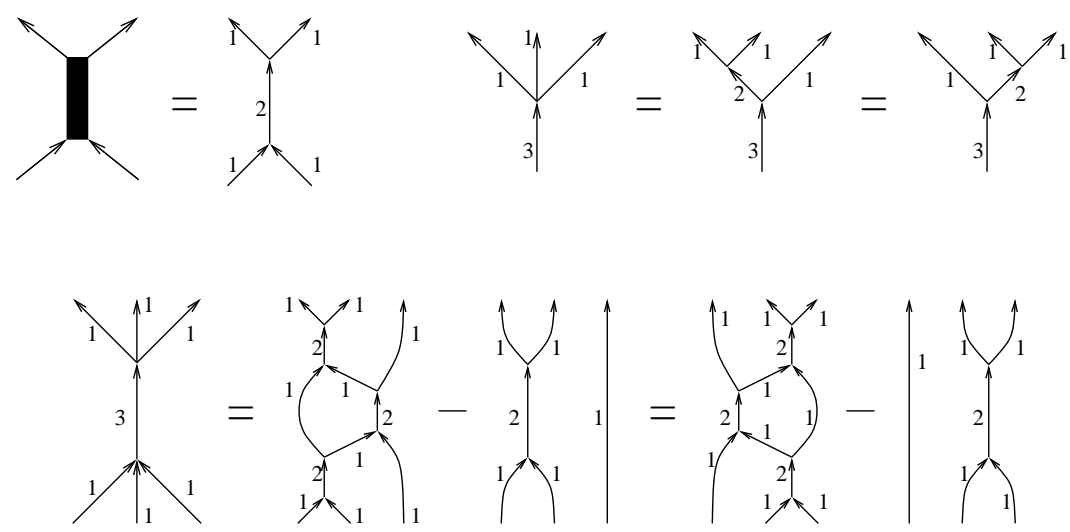

Fig. 4. Murakami-Ohtsuki-Yamada terminology and the appearance of edges labeled by 3

This calculus of planar graphs and its generalization to arbitrary exterior powers of $V$ was developed by Murakami, Ohtsuki and Yamada [MOY]. Oriented edges of graphs in their calculus carry labels from 1 to $n-1$ that 
denote fundamental weights of $\operatorname{sl}(n)$. The part of their calculus that we use deals only with edges labeled by 1 and 2 . In our notation we omit these labels; instead, we indicate edges labeled by 2 by wide lines (see Figure 4, top left). Consistency of Figure 3 relations is shown in [MOY].

The last relation in Figure 3 can be rewritten to introduce edges labeled by 3 (see Figure 4), and to say that the difference of certain two endomorphisms of $V^{\otimes 3}$ is a multiple of the projection onto the irreducible summand $\Lambda^{3} V$.

The polynomial invariant $P_{n}(L)$ of an oriented link $L$ can be computed by choosing a plane diagram $D$ of $L$, resolving each crossing as shown in Figure 2 and summing $P_{n}(\Gamma)$ weighted by powers of $q$ over all resolutions $\Gamma$ of $D$ :

$$
P_{n}(L)=P_{n}(D):=\sum_{\text {resolutions } \Gamma} q^{\alpha(\Gamma)} P_{n}(\Gamma),
$$

with $\alpha(\Gamma)$ determined by Figure 2 rules. Independence from the choice of $D$ follows from the equations in Figure 3. They imply that $P_{n}\left(D_{1}\right)=P_{n}\left(D_{2}\right)$ whenever $D_{1}$ and $D_{2}$ are related by a Reidemeister move.

To construct the homology theory $H_{n}$, we first categorify $P_{n}(\Gamma)$, by defining in a rather roundabout way a graded $\mathbb{Q}$-vector space $H(\Gamma)=$ $\bigoplus_{j \in \mathbb{Z}} H^{j}(\Gamma)$ such that

$$
P_{n}(\Gamma)=\sum_{j \in \mathbb{Z}} \operatorname{dim}_{\mathbb{Q}} H^{j}(\Gamma) q^{j}
$$

With $\Gamma$ we associate a 2-periodic complex $C(\Gamma)$ of graded $\mathbb{Q}$-vector spaces

$$
C^{0}(\Gamma) \rightarrow C^{1}(\Gamma) \rightarrow C^{0}(\Gamma)
$$

and define $H(\Gamma)$ as the degree $i$ cohomology of this complex, where $i$ is the parity of the number of components of link $L$. The construction of $C(\Gamma)$ is based on the notion of a matrix factorization. An $(R, w)$-factorization $M$ over a commutative ring $R$ (where $w \in R$ ) consists of two free $R$-modules and two $R$-module maps

$$
M^{0} \stackrel{d}{\longrightarrow} M^{1} \stackrel{d}{\longrightarrow} M^{0}
$$

such that $d^{2}(m)=w m$ for any $m \in M$. The case most commonly considered in the literature is when $R$ is the ring of power series, and $w$ satisfies a certain nondegeneracy assumption (that the quotient $R /(w)$ is an isolated singularity). Such an $M$ is called a matrix factorization. When $w$ is homogeneous, one can switch from power series to polynomials.

Matrix factorizations appeared in commutative algebra in early and mideighties $[\mathrm{E} 1],[\mathrm{B}],[\mathrm{Kn}],[\mathrm{S}],[\mathrm{BEH}]$ in the study of isolated hypersurface singularities, and much more recently in string theory, as boundary conditions for strings in Landau-Ginzburg models [KL1-3]. 
The tensor product $M \otimes_{R} N$ of an $(R, w)$-factorization $M$ and an $(R,-w)$ factorization $N$ is a 2-periodic complex of $R$-modules, with well-defined cohomology. More generally, given a finite set $\left\{w_{1}, \ldots, w_{m}\right\}$ of elements of $R$ that sum to zero, and an $\left(R, w_{i}\right)$-factorization $M_{i}$ for each $i$, the tensor product

$$
M_{1} \otimes_{R} \cdots \otimes_{R} M_{m}
$$

is a 2-periodic complex and its cohomology $H\left(\bigotimes_{i} M_{i}\right)$ is a $\mathbb{Z}_{2}$-graded $R$ module.

Starting with a resolution $\Gamma$ of a link diagram, we denote by $E$ the set of oriented edges of $\Gamma$ and by $R$ the ring of polynomials in $x_{j}, j \in E$. We give each $x_{j}$ degree 2, making $R$ graded. Assume for simplicity that $\Gamma$ has no oriented loops and each wide edge of $\Gamma$ borders exactly four oriented edges (no oriented edge shares both endpoints with the same wide edge). Let $T$ be the set of wide edges. Choosing a $t \in T$, denote the oriented edges at $t$ by $1,2,3,4$ (we think of $1,2,3,4$ as elements of $E$ ) so that the four corresponding variables are $x_{1}, x_{2}, x_{3}, x_{4}$ (see Figure 5 ).

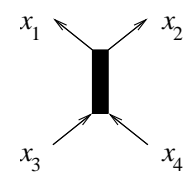

Fig. 5. Near a wide edge

Assign the polynomial

$$
w_{t}=x_{1}^{n+1}+x_{2}^{n+1}-x_{3}^{n+1}-x_{4}^{n+1}
$$

to the edge $t$. This polynomial lies in the ideal generated by $x_{1}+x_{2}-x_{3}-x_{4}$ and $x_{1} x_{2}-x_{3} x_{4}$ (since $x^{n+1}+y^{n+1}$ is a polynomial $g(x+y, x y)$ in $x+y$ and $x y$ ). Therefore, we can write

$$
w_{t}=\left(x_{1}+x_{2}-x_{3}-x_{4}\right) u_{1}+\left(x_{1} x_{2}-x_{3} x_{4}\right) u_{2}
$$

for some polynomials $u_{1}, u_{2}$. The latter are not uniquely defined, but the indeterminacy is easy to describe. We choose

$$
\begin{aligned}
& u_{1}=\frac{x_{1}^{n+1}+x_{2}^{n+1}-g\left(x_{3}+x_{4}, x_{1} x_{2}\right)}{x_{1}+x_{2}-x_{3}-x_{4}}, \\
& u_{2}=\frac{g\left(x_{3}+x_{4}, x_{1} x_{2}\right)-x_{3}^{n+1}-x_{4}^{n+1}}{x_{1} x_{2}-x_{3} x_{4}} .
\end{aligned}
$$

Let $C_{t}$ be the tensor product of the factorizations

$$
R \stackrel{x_{1}+x_{2}-x_{3}-x_{4}}{\longrightarrow} R \stackrel{u_{1}}{\longrightarrow} R
$$

and

$$
R \stackrel{x_{1} x_{2}-x_{3} x_{4}}{\longrightarrow} R \stackrel{u_{2}}{\longrightarrow} R
$$


Then $C_{t}$ is an $\left(R, w_{t}\right)$-factorization. Define $C(\Gamma)$ to be the tensor product of the $C_{t}$ over all wide edges $t$,

$$
C(\Gamma):=\bigotimes_{t \in T} C_{t} .
$$

The square of the differential in $C(\Gamma)$ is the sum of $w_{t}$ over all wide edges $t$,

$$
d^{2}=\sum_{t} w_{t}=0 .
$$

The sum is 0 , since for each oriented edge $i$ the term $x_{i}^{n+1}$ appears twice in the sum, once with positive and once with negative sign. We see that $C(\Gamma)$ is a 2-periodic complex of $\mathbb{Q}$-vector spaces.

If, in addition, $\Gamma$ contains $k$ oriented loops, to define $C(\Gamma)$ we tensor the product of $C_{t}$ 's with $k$ copies of the vector space $\mathrm{H}^{*}\left(\mathbb{C P}^{n-1}, \mathbb{Q}\right)$ and, if $k$ is odd, shift the complex. The shift $M\langle 1\rangle$ of a factorization $M^{0} \rightarrow M^{1} \rightarrow M^{0}$ is

$$
M^{1} \rightarrow M^{0} \rightarrow M^{1} .
$$

If, for some $t$, some variables (say, $x_{2}$ and $x_{3}$ ) belong to the same oriented edge (see Figure 6), we quotient the ring $R$ and the complex by the corresponding relation $\left(x_{2}=x_{3}\right)$.

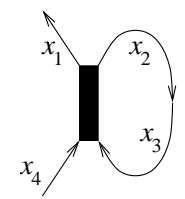

Fig. 6. $x_{2}$ and $x_{3}$ are on the same edge

We let $H(\Gamma)$ be the cohomology of $C(\Gamma)$. The latter has cohomology only in degree equal to the parity of the number of components of the link $L$. Additional internal grading in $C(\Gamma)$ coming from the grading in the polynomial ring $R$ induces a $\mathbb{Z}$-grading on $H(\Gamma)$. This, in the nutshell, is how we define $H(\Gamma)$, which serve as building blocks for complexes $C(D)$ assigned to plane diagrams $D$.

In this paper we work in greater generality, first by allowing graphs that lie inside a disc and have points on its boundary. To such a graph we assign a factorization, rather than a 2-periodic complex. Second, we place one or more marks on each oriented edge, with variables $x_{i}$ assigned to the marks, rather than just to the edges (see Figure 7). Boundary points also count as marks. Let $E$ be the set of marks and $R$ the ring of polynomials in variables $x_{i}$ for $i \in E$.

To an arc bounded by marks $x_{i}, x_{j}$ and oriented from $x_{j}$ to $x_{i}$ we assign the factorization $L_{j}^{i}$ : 


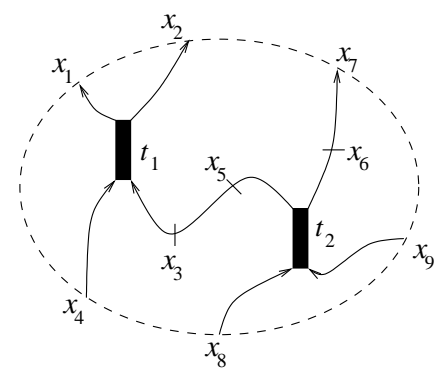

Fig. 7. A graph with boundary points and marks

$$
R \stackrel{\pi_{i j}}{\longrightarrow} R \stackrel{x_{i}-x_{j}}{\longrightarrow} R, \quad \text { where } \quad \pi_{i j}=\frac{x_{i}^{n+1}-x_{j}^{n+1}}{x_{i}-x_{j}} .
$$

We then form the tensor product $C(\Gamma)$ of the factorizations $C_{t}$ over all wide edges $t$, and factorizations $L_{j}^{i}$ over all arcs. Now we ignore the variables $x_{i}$ assigned to internal marks, and view $C(\Gamma)$ as an $\left(R^{\prime}, w\right)$-factorization, with $R^{\prime}$ being the ring of polynomials in $x_{i}$ over all boundary points $i$, and

$$
w=\sum_{i} \pm x_{i}^{n+1}
$$

with signs determined by the orientation of $\Gamma$ near boundary points. We prove that, in the homotopy category of factorizations, $C(\Gamma)$ does not depend on how we place internal marks.

For example, if $\Gamma$ is as in Figure 7 , to the wide edges $t_{1}$ and $t_{2}$ we assign polynomials $w_{t_{1}}$ and $w_{t_{2}}$ in variables $x_{1}, x_{2}, x_{3}, x_{4}$ and $x_{5}, x_{6}, x_{8}, x_{9}$, respectively, and factorizations $C_{t_{1}}, C_{t_{2}}$. Form the tensor product factorization

$$
C(\Gamma)=C_{t_{1}} \otimes_{R} C_{t_{2}} \otimes_{R} L_{5}^{3} \otimes_{R} L_{6}^{7}
$$

where $R$ is the ring of polynomials in $x_{1}, \ldots, x_{9}$. We then ignore the variables $x_{3}, x_{5}, x_{6}$ that came from internal marks, and treat $C(\Gamma)$ as an $\left(R^{\prime}, w\right)$ factorization with

$$
w=x_{1}^{n+1}+x_{2}^{n+1}-x_{4}^{n+1}+x_{7}^{n+1}-x_{8}^{n+1}-x_{9}^{n+1},
$$

and $R^{\prime}$ being the ring of polynomials in the "boundary" variables $x_{1}, x_{2}$, $x_{4}, x_{7}, x_{8}, x_{9}$. As an $R^{\prime}$-module, $C(\Gamma)$ is free but has infinite rank. However, by stripping off contractible summands, $C(\Gamma)$ can be reduced to a finite rank factorization.

We prove that the factorizations $C(\Gamma)$ have direct sum decompositions that mimic skein relations in Figure 3, and define homomorphisms $\chi_{0}$ and $\chi_{1}$ between factorizations $C\left(\Gamma^{0}\right)$ and $C\left(\Gamma^{1}\right)$ in Figure 8.

Then we consider oriented tangles in a 3 -ball $B^{3}$ such that all boundary points of a tangle lie on a fixed great circle of the boundary sphere. Let $D$ be a generic projection of a tangle $L$ onto the plane of this great circle. 

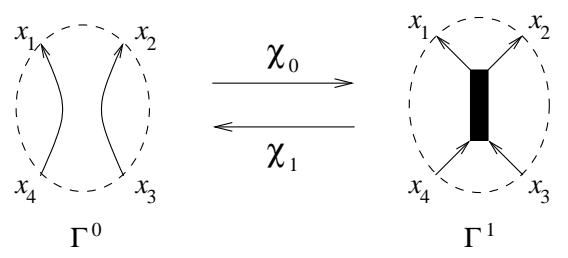

Fig. 8. Graphs $\Gamma^{0}$ and $\Gamma^{1}$

We separate crossings of $D$ into positive and negative, following the rule in Figure 45. To each crossing we assign two planar graphs (resolutions of this crossing); see Figure 9.

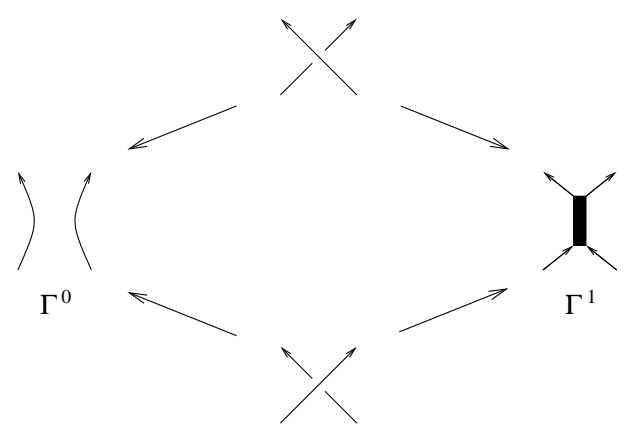

Fig. 9. Resolving crossings

To a diagram of a single crossing we assign the complex of factorizations

$$
0 \rightarrow C\left(\Gamma^{0}\right) \stackrel{\chi_{0}}{\longrightarrow} C\left(\Gamma^{1}\right) \rightarrow 0
$$

if the crossing is positive, and the complex

$$
0 \rightarrow C\left(\Gamma^{1}\right) \stackrel{\chi_{1}}{\longrightarrow} C\left(\Gamma^{0}\right) \rightarrow 0
$$

if the crossing is negative (shifts in the internal grading should be added to match powers of $q$ that appear in Figure 2 formulas). In both cases we place $C\left(\Gamma^{0}\right)$ in cohomological degree 0 .

In general, we place marks on each internal edge of $D$, form a commutative cube of factorizations $C(\Gamma)$ over all resolutions $\Gamma$ of $D$, and take the total complex $C(D)$ of the cube. Each factorization $C(\Gamma)$ has additional $\mathbb{Z}$-grading, induced by the grading of the polynomial ring $R$, and the differential is grading-preserving. If we ignore the differentials, $C(\Gamma)$ is a $\mathbb{Z} \oplus \mathbb{Z} \oplus \mathbb{Z}_{2}$-graded $R$-module.

We prove that if $D_{1}$ and $D_{2}$ are related by a Reidemeister move, then $C\left(D_{1}\right)$ and $C\left(D_{2}\right)$ are isomorphic as objects in a suitable homotopy category. Therefore, the isomorphism class of $C(D)$ in this category is an invariant of the tangle $L$. 
A cobordism $S$ between tangles $T_{0}$ and $T_{1}$ is an oriented surface properly embedded in $B^{3} \times[0,1]$ with $S \cap\left(B^{3} \times\{i\}\right)=T_{i}$, and some additional standard assumptions. $S$ can be presented by a "movie" - a sequence of plane diagrams of its cross-sections with $B^{3} \times k$ for various $k \in[0,1]$. The sequence starts with a diagram $D_{0}$ of $T_{0}$ and ends with a diagram $D_{1}$ of $T_{1}$. To such a sequence we associate a homomorphism between complexes of factorizations $C\left(D_{0}\right)$ and $C\left(D_{1}\right)$ (with certain grading shifts thrown in), and show that, up to null-homotopies and multiplications by nonzero rationals, the homomorphism does not depend on the movie presentation of $S$ (as long as $D_{0}$ and $D_{1}$ are the first and last slides). In this way, for each positive $n$, we obtain an invariant of tangle cobordism (trivial invariant if $n=1$, and a variation of the one in [Kh4] if $n=2)$.

If $L$ is a link, then $C(\Gamma)$, for any resolution $\Gamma$ of $D$, is a 2-periodic complex of graded $\mathbb{Q}$-vector spaces. It has cohomology groups only in degree equal to the parity of the number of components in $L$. Thus, after removing the contractible summands, $C(\Gamma)$ reduces to a graded vector space, and $C(D)$ to a complex of graded vector spaces. Its cohomology groups

$$
H_{n}(D)=\bigoplus_{i, j \in \mathbb{Z}} H_{n}^{i, j}(D)
$$

do not depend, up to isomorphism, on the choice of the projection of $L$. The invariant of link cobordism is a homomorphism between these cohomology groups, well-defined up to rescaling by nonzero rational numbers. Due to this, $H_{n}(D)$ are canonically (up to rescaling) associated to $L$, rather than just to its diagram $D$. Thus, notation $H_{n}(L)$ is justified. The Euler characteristic of $H_{n}(L)$ is the polynomial $P_{n}(L)$ :

$$
P_{n}(L)=\sum_{i, j \in \mathbb{Z}}(-1)^{i} q^{j} \operatorname{dim}_{\mathbb{Q}} H_{n}^{i, j}(L) .
$$

$H(\Gamma)$ is the cohomology of the complex $C(\Gamma)$ which has countable dimension as a $\mathbb{Q}$-vector space. However, since $H(\Gamma)$ is finite-dimensional, it is nontrivial in finitely many degrees only, and an obvious upper bound on $|j|$ with $H^{j}(\Gamma) \neq 0$ is ne where $e$ is the number of edges in $\Gamma$ (both oriented and unoriented). The complex $C(D)$ is built out of finitely many $H(\Gamma)$, and the differential, being the signed sum of maps $\chi_{0}, \chi_{1}$, can be determined combinatorially as well. Therefore, there exists a combinatorial algorithm to find the homology groups $H_{n}(L)$, given $L$.

If $n=1$, the factorization $C_{t}$ is contractible, and any graph with a wide edge has trivial homology. The homology of a circle is $\mathbb{Q}$. This implies that $H_{1}(L) \cong \mathbb{Q}$ for any link $L$, with $\mathbb{Q}$ in bidegree $(0,0)$.

When $n=2$ and $\Gamma$ is closed (has no boundary points), its cohomology groups $H(\Gamma)$ are isomorphic to $A_{2}^{\otimes k}$, where $A_{2}$ is the cohomology ring of the 2 -sphere and $k$ the number of circles in the diagram given by deleting all 
wide edges of $\Gamma$. The equivalence of $H_{2}$ with a version of homology theory in [Kh1] follows easily from this observation. Specifically, for each link $L$ there is an isomorphism

$$
H_{2}^{i, j}(L) \cong \mathcal{H}^{i,-j}\left(L^{!}\right) \otimes_{\mathbb{Z}} \mathbb{Q},
$$

where $L^{!}$is the mirror image of $L$, and $\mathcal{H}$ is the homology theory defined in [Kh1, Section 7] (programs computing $\mathcal{H}(L)$ were implemented by Dror Bar-Natan [BN1] and Alexander Shumakovitch [Sh]).

We conjecture that $\mathrm{H}_{3}$ is isomorphic to the homology theory constructed in $[\mathrm{Kh} 3]$, after the latter is tensored with $\mathbb{Q}$. Both theories are doubly-graded, have the same Euler characteristic, and are built out of similar long exact sequences. The language of foams used in [Kh3] and $[\mathrm{R}]$ should extend from $n=3$ to all $n$ and lead to a better understanding of $H_{n}$.

Our categorification of the polynomials $P_{n}(L)$ comes with several obvious caveats:

- The homology groups $H_{n}(L)$ are finite-dimensional $\mathbb{Q}$-vector spaces rather than finitely generated abelian groups (as in [Kh1], [Kh3] for $n=2,3)$.

- Defining $H_{n}(L)$ requires a choice of a plane diagram. A more intrinsic definition would be most welcome.

- Our invariants of link and tangle cobordisms are projective (defined up to overall multiplication by a non-zero rational number).

- Reconstructing the Ozsváth-Szabó-Rasmussen theory (the $n=0$ case) using this approach would require additional ideas, lacking at the moment.

Construction of $C(\Gamma)$ involves tensoring factorizations

$$
R \stackrel{a}{\longrightarrow} R \stackrel{b}{\longrightarrow} R
$$

for various $a$ 's and $b$ 's. We do an elementary study of these tensor products in Section 2. Section 3 contains a review of matrix factorizations and their properties. In Section 4 we explain how to view factorizations as functors and present the identity functor via a factorization. These two sections also introduce general framework for diagrammatical interpretation of matrix factorizations. Section 5 treats graded factorizations and explains how to modify the material of previous sections to cover this case. Section 6 is the computational core of the paper. We define a factorization $C(\Gamma)$ assigned to a planar graph $\Gamma$, construct morphisms $\chi_{0}, \chi_{1}$ between graphs $\Gamma^{0}, \Gamma^{1}$ in Figure 8, and prove direct sum decompositions of the factorizations $C(\Gamma)$ that lift the skein relations of Figure 3 . In Section 7 we associate a complex of factorizations $C(D)$ to a plane diagram $D$ of a tangle and state Theorem 2 . This theorem, claiming the invariance of $C(D)$ under Reidemeister moves in a suitable category of complexes of factorizations, is proved in Section 8. In 
Section 9 we use matrix factorizations to construct 2-dimensional topological quantum field theories with corners. The next section deals with functoriality of our tangle invariant, extending it to tangle cobordisms. In the last section we briefly outline an approach to categorification of quantum invariants of links colored by arbitrary fundamental representations of $\operatorname{sl}(n)$.

Acknowledgments. M.K. is indebted to Ragnar-Olaf Buchweitz, Igor Burban, and Paul Seidel for introducing him to matrix factorizations, and to Igor Burban for sharing M.K.'s fascination with 2-periodic triangulated categories. L.R. would like to thank Anton Kapustin for explanations regarding topological 2-dimensional models and his work with Yi Li. We are grateful to Dror Bar-Natan and Greg Kuperberg for interesting discussions. While writing this paper we were partially supported by NSF grants DMS-0104139 and DMS-0196131.

2. The cyclic Koszul complex. Let $R$ be a Noetherian commutative ring. The Koszul complex $R\left(a_{1}, \ldots, a_{m}\right)$ associated to a sequence $a_{1}, \ldots, a_{m}$, where $a_{i} \in R$, is the tensor product (over $R$ ) of complexes

$$
0 \rightarrow R \stackrel{a_{i}}{\longrightarrow} R \rightarrow 0
$$

for $i=1, \ldots, m$. A sequence $\left(a_{1}, \ldots, a_{m}\right)$ is called $R$-regular if $a_{i}$ is not a zero divisor in the quotient ring $R /\left(a_{1}, \ldots, a_{i-1}\right) R$ for each $i=1, \ldots, m$, and $R /\left(a_{1}, \ldots, a_{m}\right) R \neq 0$. If the sequence is $R$-regular, its Koszul complex has cohomology in the rightmost degree only. For the converse to be true it suffices for $R$ to be local (see [E1, Section 17]).

The Koszul complex can be written in a more intrinsic way. Let $N$ be a free $R$-module of rank $m$ and $a: R \rightarrow N$ an $R$-module homomorphism. The Koszul complex of $a$ is the complex of exterior powers of $N$ :

$$
0 \rightarrow R \rightarrow N \rightarrow \Lambda^{2} N \rightarrow \Lambda^{3} N \rightarrow \cdots,
$$

with the differential being the exterior product with $a(1) \in N$. Choosing a basis of $N$ and writing $a(1)$ in coordinates as $\left(a_{1}, \ldots, a_{m}\right)$, we recover the Koszul complex of this sequence.

Definition 1. Let $w \in R$. An $(R, w)$-duplex consists of two $R$-modules $M^{0}, M^{1}$ and module maps

$$
M^{0} \stackrel{d}{\longrightarrow} M^{1} \stackrel{d}{\longrightarrow} M^{0}
$$

such that $d^{2}=w$ (as endomorphisms of $M^{0}$ and $M^{1}$ ).

In other words, $d^{2} m=w m$ for any $m \in M^{0} \oplus M^{1}$. "Duplex" is a term from [FKS], where it was used in a more general situation, with $R$ a (possibly noncommutative) ring and $w$ its central element. 
A homomorphism $f: M \rightarrow N$ of duplexes is a pair of homomorphisms $f^{0}: M^{0} \rightarrow N^{0}$ and $f^{1}: M^{1} \rightarrow N^{1}$ that make the diagram below commute.

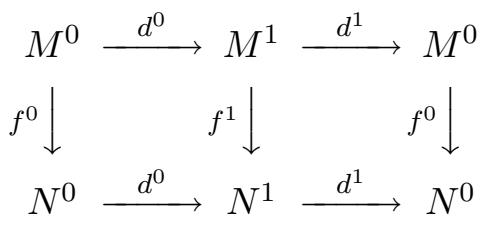

The category of $(R, w)$-duplexes and duplex homomorphisms is abelian.

A homotopy $h$ between homomorphisms $f, g: M \rightarrow N$ of duplexes is a pair of maps $h^{i}: M^{i} \rightarrow N^{i-1}$ (with indices understood modulo 2) such that

$$
f-g=h d_{M}+d_{N} h .
$$

Null-homotopic morphisms constitute an ideal in the category of duplexes. We call the quotient category by this ideal the category of duplexes up to homotopy, or simply the homotopy category of duplexes. This category is triangulated. We denote the shift functor by $\langle 1\rangle$.

An $(R, 0)$-duplex $M$ is equivalent to a 2 -complex (periodic complex with period two) of $R$-modules. We denote the cohomology of an $(R, 0)$-duplex $M$ by $H(M) \cong H^{0}(M) \oplus H^{1}(M)$.

Definition 2. A factorization (or matrix factorization) is an $(R, w)$ duplex such that $M^{0}, M^{1}$ are free $R$-modules.

The homotopy category of $(R, w)$-factorizations is triangulated.

To a pair of elements $a, b \in R$ we associate the $(R, a b)$-factorization

$$
R \stackrel{a}{\longrightarrow} R \stackrel{b}{\longrightarrow} R
$$

denoted $\{a, b\}$.

If $\mathbf{a}=\left(a_{1}, \ldots, a_{m}\right)$ and $\mathbf{b}=\left(b_{1}, \ldots, b_{m}\right)$ are two sequences of elements of $R$, we consider the tensor product factorization $\{\mathbf{a}, \mathbf{b}\}:=\otimes_{i}\left\{a_{i}, b_{i}\right\}$, where the tensor product is over $R$. We call $\{\mathbf{a}, \mathbf{b}\}$ the Koszul factorization of the pair $(\mathbf{a}, \mathbf{b})$. We say that a pair $(\mathbf{a}, \mathbf{b})$ is orthogonal if

$$
\mathbf{a b}:=\sum_{i} a_{i} b_{i}=0 .
$$

The tensor product $\{\mathbf{a}, \mathbf{b}\}$ is a 2-complex iff $(\mathbf{a}, \mathbf{b})$ is orthogonal, in which case we call it the periodic (or cyclic) Koszul complex of $(\mathbf{a}, \mathbf{b})$.

REMARK. We allow the case $m=0$. Then $\mathbf{a}$ and $\mathbf{b}$ are empty sequences, $w=0$, and the Koszul factorization is $R \rightarrow 0 \rightarrow R$.

REMARK. Factorizations $\{\mathbf{a}, \mathbf{b}\}$ were defined in $[\mathrm{BGS}]$ and used there in the classification of finite CM-type singularities. 
Proposition 1. If the entries of $\mathbf{a}$ and $\mathbf{b}$ generate $R$ as an $R$-module, then the factorization $\{\mathbf{a}, \mathbf{b}\}$ is contractible (its identity endomorphism is null-homotopic).

Proof. Write $1=\sum_{i}\left(a_{i}^{\prime} a_{i}+b_{i}^{\prime} b_{i}\right)$. The pair $\left(\mathbf{a}^{\prime}, \mathbf{b}^{\prime}\right)$, for $\mathbf{a}^{\prime}=\left(a_{1}^{\prime}, \ldots, a_{m}^{\prime}\right)$ and $\mathbf{b}=\left(b_{1}^{\prime}, \ldots, b_{m}^{\prime}\right)$, defines a homotopy between the identity and the zero endomorphism of $\{\mathbf{a}, \mathbf{b}\}$.

COROLlary 1. If $(\mathbf{a}, \mathbf{b})$ is orthogonal and the entries of $\mathbf{a}$ and $\mathbf{b}$ generate $R$ as an $R$-module, then the 2-complex $\{\mathbf{a}, \mathbf{b}\}$ is acyclic.

Let $I_{\mathbf{a}, \mathbf{b}} \subset R$ be the ideal of $R$ generated by the entries of $\mathbf{a}$ and $\mathbf{b}$.

Proposition 2. $H(\{\mathbf{a}, \mathbf{b}\})$ is an $R / I_{\mathbf{a}, \mathbf{b}}$-module.

Proof. Multiplications by $a_{i}$ and $b_{i}$ are null-homotopic endomorphisms of $\left\{a_{i}, b_{i}\right\}$, and therefore of $\{\mathbf{a}, \mathbf{b}\}$.

Let $\mathbf{a}^{i}, \mathbf{b}^{i}$ be the sequences obtained from $\mathbf{a}$ and $\mathbf{b}$ by omitting $a_{i}$ and $b_{i}$, respectively. The factorization $\{\mathbf{a}, \mathbf{b}\}$ is the "total factorization" of the bifactorization

$$
\left\{\mathbf{a}^{i}, \mathbf{b}^{i}\right\} \stackrel{a_{i}}{\longrightarrow}\left\{\mathbf{a}^{i}, \mathbf{b}^{i}\right\} \stackrel{b_{i}}{\longrightarrow}\left\{\mathbf{a}^{i}, \mathbf{b}^{i}\right\} .
$$

If $b_{i}$ is a nonzerodivisor in $R$, the second map is injective (as a map between $R$-modules), and the direct sum of the middle $\left\{\mathbf{a}^{i}, \mathbf{b}^{i}\right\}$ and its image under the differential is contractible. Furthermore, suppose that $(\mathbf{a}, \mathbf{b})$ is orthogonal. Then the quotient of $\{\mathbf{a}, \mathbf{b}\}$ by this contractible subcomplex is isomorphic to $\left\{\mathbf{a}^{i}, \mathbf{b}^{i}\right\}_{R^{\prime}}$, the periodic Koszul complex of the pair $\left(\mathbf{a}^{i}, \mathbf{b}^{i}\right)$ in the quotient ring $R^{\prime}:=R /\left(b_{i}\right)$. The quotient map induces an isomorphism on cohomology,

$$
H(\{\mathbf{a}, \mathbf{b}\}) \cong H\left(\left\{\mathbf{a}^{i}, \mathbf{b}^{i}\right\}_{R^{\prime}}\right),
$$

which is an isomorphism of $R^{\prime}$-modules.

Corollary 2. If $(\mathbf{a}, \mathbf{b})$ is orthogonal and $\mathbf{b}$ is $R$-regular then

$$
H^{0}(\{\mathbf{a}, \mathbf{b}\}) \cong R /\left(b_{1}, \ldots, b_{m}\right) \text { and } H^{1}(\{\mathbf{a}, \mathbf{b}\})=0 .
$$

Likewise, if $a_{i}$ is a nonzerodivisor in $R$ and $(\mathbf{a}, \mathbf{b})$ is orthogonal, then the quotient map

$$
\{\mathbf{a}, \mathbf{b}\}\langle 1\rangle \rightarrow\left\{\mathbf{a}^{i}, \mathbf{b}^{i}\right\}_{R /\left(a_{i}\right)}
$$

induces an isomorphism on cohomology.

Motivated in part by Corollary 2, we introduce

DeFinition 3. An orthogonal pair $(\mathbf{a}, \mathbf{b})$ is called homologically $R$-regular if $H^{0}(\{\mathbf{a}, \mathbf{b}\}) \neq 0$ and $H^{1}(\{\mathbf{a}, \mathbf{b}\})=0$.

If $(\mathbf{a}, \mathbf{b})$ is homologically $R$-regular then $I_{\mathbf{a}, \mathbf{b}}$ is a proper ideal in $R$. 
EXAMPLE. Let $m=1$. An orthogonal pair $\left(a_{1}, b_{1}\right)$ is homologically $R$ regular iff $a_{1} R=\operatorname{Ann}\left(b_{1}\right)$ and $b_{1} R$ is a proper subset of $\operatorname{Ann}\left(a_{1}\right)$. Furthermore, when $R$ is a domain, $\left(a_{1}, b_{1}\right)$ is homologically $R$-regular iff $a_{1}=0$ and $b_{1}$ is not invertible.

EXAmple. Let $\mathbf{0}=(0, \ldots, 0)$. The pair $(\mathbf{0}, \mathbf{b})$ is homologically $R$-regular iff the Koszul complex of $\mathbf{b}$ has cohomology in the rightmost degree only.

Suppose there is a subset $J \subset\{1, \ldots, m\}$ with even number of elements such that the sequence $\left(h_{1}, \ldots, h_{m}\right)$ is $R$-regular, where $h_{i}=a_{i}$ if $i \in J$ and $h_{i}=b_{i}$ otherwise. Then $(\mathbf{a}, \mathbf{b})$ is homologically $R$-regular and $H^{0}(\{\mathbf{a}, \mathbf{b}\}) \cong$ $A /\left(h_{1}, \ldots, h_{m}\right)$.

We can likewise define the notion of homologically $M$-regular pair (a, b) for any $R$-module $M$, and even for any $(R, w)$-duplex $M$. For the latter, we require $\mathbf{a b}=-w, H^{0}\left(M \otimes_{R}\{\mathbf{a}, \mathbf{b}\}\right) \neq 0$ and $H^{1}\left(M \otimes_{R}\{\mathbf{a}, \mathbf{b}\}\right)=0$.

Let $N$ be a finitely-generated free $R$-module and $\alpha: R \rightarrow N, \beta: N \rightarrow R$ be $R$-module maps. Consider the factorization $\{\alpha, \beta\}$ given by

$$
\Lambda^{\text {even }} N \stackrel{\wedge \alpha+\neg \beta}{\longrightarrow} \Lambda^{\text {odd }} N \stackrel{\wedge \alpha+\neg \beta}{\longrightarrow} \Lambda^{\text {even }} N
$$

where $\wedge \alpha$ is the wedge product with $\alpha(1)$,

$$
\wedge \alpha: \Lambda^{i} N \rightarrow \Lambda^{i+1} N
$$

$\neg \beta$ is the contraction with $\beta$,

$$
\neg \beta: \Lambda^{i} N \rightarrow \Lambda^{i-1} N,
$$

and

$$
\Lambda^{\text {even }} N=\bigoplus_{i} \Lambda^{2 i} N, \quad \Lambda^{\text {odd }} N=\bigoplus_{i} \Lambda^{2 i+1} N .
$$

$\{\alpha, \beta\}$ is a 2-complex iff $\beta \alpha=0$, in which case we say that $\alpha$ and $\beta$ are orthogonal. Choosing a basis of $N$, we can write $\alpha=\mathbf{a}=\left(a_{1}, \ldots, a_{m}\right)^{T}$ and $\beta=\mathbf{b}=\left(b_{1}, \ldots, b_{m}\right)$. Then $\{\alpha, \beta\}$ is isomorphic to $\{\mathbf{a}, \mathbf{b}\}$.

Thus, with each element $\gamma \in N \oplus N^{*}$ we associate a factorization

$$
\{\gamma\}:=\{\alpha, \beta\},
$$

where $\gamma=\alpha+\beta$ and $\alpha \in N, \beta \in N^{*}$.

Suppose $g: N \rightarrow N$ is an automorphism of the $R$-module $N$. Write the composition $\beta \alpha$ as $\beta \gamma^{-1} \gamma \alpha$. The factorizations $\{\alpha, \beta\}$ and $\left\{\gamma \alpha, \beta \gamma^{-1}\right\}$ are isomorphic, and $G L(N, R)$ acts on the set of pairs $(\alpha, \beta)$ preserving the isomorphism classes of factorizations $\{\alpha, \beta\}$.

We let the group $H=\left(\mathbb{Z}_{2}\right)^{\times m}$ act on pairs by permuting $a_{i}$ with $a_{i}$ in $\left(a_{1}, a_{1}\right), \ldots,\left(a_{m}, a_{m}\right)$ for $1 \leq i \leq m$.

For $\sigma \in H$, the factorizations $\{\mathbf{a}, \mathbf{b}\}$ and $\{\sigma(\mathbf{a}, \mathbf{b})\}$ are isomorphic if $\sigma$ is an even permutation in $H \subset \mathbb{S}_{2 m}$. If $\sigma$ is odd, the factorization $\{\sigma(\mathbf{a}, \mathbf{b})\}$ is isomorphic to $\{\mathbf{a}, \mathbf{b}\}\langle 1\rangle$. 
The natural $R$-linear pairing between $N^{*}$ and $N$ induces a symmetric bilinear form on $N \oplus N^{*}$. Let $G^{\prime} \subset G L\left(N \oplus N^{*}, R\right)$ be the subgroup generated by $G L(N, R)$ and $H$ that act as described above. The action of $G^{\prime}$ preserves the inner product on $N \oplus N^{*}$ (with values in $R$ ). Let $G$ be the subgroup of $G^{\prime}$ that consists of all products $g_{1} \sigma_{1} \ldots g_{r} \sigma_{r}$ of elements from $G L(N, R)$ and $H$ (over all $r$ ) such that $\sigma_{1} \ldots \sigma_{r}$ is an even permutation.

The following result is clear.

Proposition 3. $\{\gamma\}$ and $\{g \gamma\}$ are isomorphic factorizations for any $g \in G$ and $\gamma \in N \oplus N^{*}$.

The cyclic Koszul complex can be thought of as the square root of the Koszul complex. Namely,

$$
\operatorname{Hom}_{R}(\{\mathbf{a}, \mathbf{b}\},\{\mathbf{a}, \mathbf{b}\}) \cong\{\mathbf{a}, \mathbf{b}\} \otimes_{R}\{-\mathbf{b}, \mathbf{a}\} \cong R(\mathbf{a}, \mathbf{b}),
$$

where $R(\mathbf{a}, \mathbf{b})$ is the Koszul complex of the length $2 m$ sequence given by concatenating $\mathbf{a}$ and $\mathbf{b}$, with the grading collapsed from $\mathbb{Z}$ to $\mathbb{Z}_{2}$.

\section{Potentials, isolated singularities, and matrix factorizations}

Potentials and their Jacobian algebras. We start with a finite set $x=$ $\left\{x_{1}, \ldots, x_{k}\right\}$ of variables and let

$$
R=\mathbb{Q}[[x]]:=\mathbb{Q}\left[\left[x_{1}, \ldots, x_{k}\right]\right]
$$

be the algebra of power series in $x_{1}, \ldots, x_{k}$ with rational coefficients. Denote by $\mathfrak{m}$ the unique maximal ideal in $R$ (generated by $x_{1}, \ldots, x_{k}$ ).

We say that a polynomial $w=w(x) \in \mathfrak{m}^{2}$ is a potential if the algebra $R / I_{w}$ is finite-dimensional, where $I_{w} \subset R$ is the ideal generated by the partial derivatives $\partial_{i} w:=\partial_{x_{i}} w$. Any polynomial $w \in R$ defines an algebraic map $\mathbb{C}^{n} \rightarrow \mathbb{C}$. This map has an isolated singularity at 0 iff $w$ is a potential.

A polynomial $w \in \mathfrak{m}^{2}$ is a potential iff $\partial_{1} w, \ldots, \partial_{k} w$ is a regular sequence in $R$, that is, $\partial_{i} w$ is not a zero divisor in $R /\left(\partial_{1} w, \ldots, \partial_{i-1} w\right) R$ for each $1 \leq i \leq k$.

The quotient algebra $R_{w}:=R / I_{w}$ is called the local algebra of the singularity and the Jacobian (or Milnor) algebra of $w$. Its dimension is the Milnor number of the singularity.

Proposition 4. The Jacobian algebra $R_{w}$ is symmetric.

A nondegenerate $\mathbb{C}$-linear trace $\operatorname{Tr}: R_{w} \otimes_{\mathbb{Q}} \mathbb{C} \rightarrow \mathbb{C}$ is given by the residue formula

$$
\operatorname{Tr}(a)=\frac{1}{(2 \pi i)^{k}} \int_{\left|\partial_{j} w\right|=\varepsilon} \frac{a d x_{1} \ldots d x_{k}}{\partial_{1} w \ldots \partial_{k} w}
$$


(proved in [GH, Chapter 5] and [AGV]). Therefore, the $\mathbb{C}$-algebra $R_{w} \otimes_{\mathbb{Q}} \mathbb{C}$ is symmetric. From [NN, Theorem 5] we deduce that $R_{w}$ is a symmetric $\mathbb{Q}$-algebra.

Exterior sum of potentials. Let $x$ and $y$ be two disjoint finite sets of variables. Define the exterior sum of potentials $w_{1}(x)$ and $w_{2}(y)$ as the potential $w_{1}(x)+w_{2}(y)$ in variables $x$ and $y$. The Jacobian algebra of the exterior sum of potentials is the tensor product of the Jacobian algebras:

$$
R_{w_{1}(x)+w_{2}(y)} \cong R_{w_{1}(x)} \otimes_{\mathbb{Q}} R_{w_{2}(y)} .
$$

Matrix factorizations. A matrix factorization of a potential $w$ is a collection of two free $A$-modules $M^{0}, M^{1}$ and two module maps $d^{0}: M^{0} \rightarrow M^{1}$ and $d^{1}: M^{1} \rightarrow M^{0}$ such that

$$
d^{0} d^{1}=w \cdot \operatorname{Id}_{M^{1}}, \quad d^{1} d^{0}=w \cdot \operatorname{Id}_{M^{0}} .
$$

We will often write a matrix factorization as

$$
M^{0} \stackrel{d}{\longrightarrow} M^{1} \stackrel{d}{\longrightarrow} M^{0} .
$$

The modules $M^{0}$ and $M^{1}$ are not required to have finite rank. Since $w$ is invertible in the field of fractions $\mathbb{Q}((x))$ of $R$, and the rank of a free $R$-module $N$ can be defined as the dimension of the $\mathbb{Q}((x))$-vector space $N \otimes_{R} \mathbb{Q}((x))$, we see that $M^{0}$ and $M^{1}$ have equal ranks (when the ranks are finite). In general, $\operatorname{rk}\left(M^{0}\right)=\operatorname{rk}\left(M^{1}\right)$, understood as equality of ordinals. We call $\operatorname{rk}\left(M^{0}\right)$ the rank of $M$.

A homomorphism $f: M \rightarrow N$ of factorizations is a pair of homomorphisms $f^{0}: M^{0} \rightarrow N^{0}$ and $f^{1}: M^{1} \rightarrow N^{1}$ that make the diagram below commute.

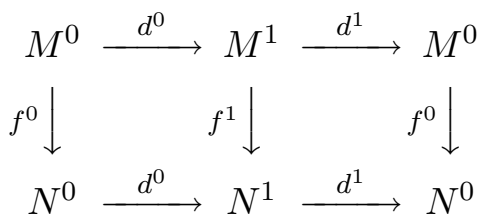

Denote the set of homomorphisms from $M$ to $N$ by $\operatorname{Hom}_{\mathrm{MF}}(M, N)$. It is an $R$-module, with the action $a\left(f^{0}, f^{1}\right)=\left(a f^{0}, a f^{1}\right)$ for $a \in R$.

Let $\mathrm{MF}_{w}^{\text {all }}$ be the category whose objects are matrix factorizations and morphisms are homomorphisms of factorizations. This category is additive and $R$-linear. Direct sum of matrix factorizations $M$ and $N$ is defined in the obvious way:

$$
(M \oplus N)^{i}=M^{i} \oplus N^{i}, \quad d_{M \oplus N}^{i}=d_{M}^{i}+d_{N}^{i} .
$$

The shifted factorization $M\langle 1\rangle$ is given by

$$
M\langle 1\rangle^{i}=M^{i+1}, \quad d_{M\langle 1\rangle}^{i}=-d_{M}^{i+1}, \quad i=0,1 \bmod 2 .
$$

$\langle 1\rangle$ is a functor in $\mathrm{MF}_{w}^{\text {all }}$ whose square is the identity, and $\langle 2\rangle \cong \mathrm{Id}$. 
EXAMPLE. If the set of variables $x$ is empty, then $R=\mathbb{Q}, f=0$, and a matrix factorization is a pair of vector spaces and a pair of maps between them such that their composition in any order is 0 . We call such data a 2-complex (short for 2-periodic complex).

Matrix factorizations first appeared in the work of David Eisenbud [E1], who related them to maximal Cohen-Macaulay modules over isolated hypersurface singularities (see also [Y1, Chapter 7], [S], and references therein). A module $N$ over a commutative Noetherian local ring is called maximal Cohen-Macaulay if the depth of $N$ equals the Krull dimension of the ring. Given a matrix factorization $M$, the $R /(f)$-module Coker $\left(d^{1}\right)$ is maximal Cohen-Macaulay, and any maximal Cohen-Macaulay module over $R /(f)$ can be presented in this way (see the above references for details). For a reader-friendly treatment of the background concepts leading to maximal Cohen-Macaulay modules we suggest the book [E2].

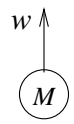

Fig. 10. A matrix factorization

Graphical notation. We denote a matrix factorization $M$ with potential $w$ as in Figure 10. We allow reversing orientation of the arc attached to $M$ simultaneously with changing potential $w$ to $-w$ (see Figure 11).
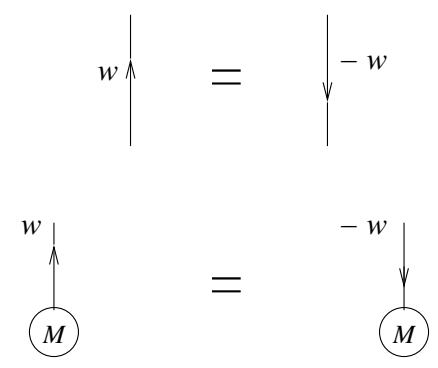

Fig. 11. Orientation reversal

If $w$ is the exterior sum of potentials, we may introduce several arcs at $M$, one for each summand (see Figure 12).

Factorizations of finite rank. Let $\mathrm{MF}_{w}^{\prime}$ be the category of finite rank matrix factorizations. It is a full subcategory in $\mathrm{MF}_{w}^{\text {all }}$. Choose bases in free $R$-modules $M^{0}$ and $M^{1}$. The maps $d^{0}, d^{1}$ can be written as $m \times m$ matrices $D_{0}, D_{1}$ with coefficients in $R$. These matrices satisfy the equations

$$
D_{0} D_{1}=w \cdot \mathrm{Id}, \quad D_{1} D_{0}=w \cdot \mathrm{Id}
$$



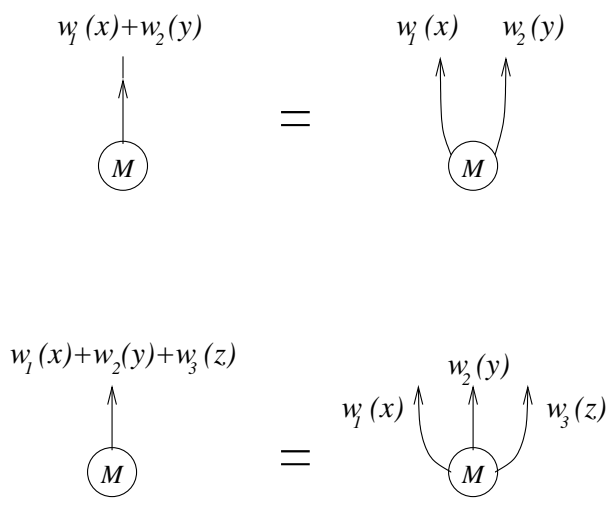

Fig. 12. Diagrams for a factorization over exterior sum of potentials

(of course, any one of these equations implies the other). Alternatively, we can describe this factorization by a $2 m \times 2 m$ matrix with off-diagonal blocks $D_{0}$ and $D_{1}$ :

$$
D=\left(\begin{array}{cc}
0 & D_{1} \\
D_{0} & 0
\end{array}\right), \quad D^{2}=w \cdot \operatorname{Id}
$$

Matrix description of objects in $\mathrm{MF}_{w}^{\prime}$ extends to infinite rank factorizations. The matrices $D_{0}$ and $D_{1}$ then have infinite rank, but each of their columns has only finitely many nonzero entries.

If factorizations $M$ and $N$ are written in matrix form, $M=\left(D_{0}, D_{1}\right)$, and $N=\left(D_{0}^{\prime}, D_{1}^{\prime}\right)$, a homomorphism $f: M \rightarrow N$ is a pair of matrices $\left(F_{0}, F_{1}\right)$ such that $F_{1} D_{0}=D_{0}^{\prime} F_{0}$ and $F_{0} D_{1}=D_{1}^{\prime} F_{1}$ (note, though, that the two equations are equivalent).

Homotopies of factorizations. A homotopy $h$ between maps $f, g: M \rightarrow$ $N$ of factorizations is a pair of maps $h^{i}: M^{i} \rightarrow N^{i-1}$ such that

$$
f-g=h d_{M}+d_{N} h .
$$

Null-homotopic morphisms constitute an ideal in the category $\mathrm{MF}_{w}^{\text {all }}$. Let $\mathrm{HMF}_{w}^{\text {all }}$ be the quotient category by this ideal. It has the same objects as $\mathrm{MF}_{w}^{\text {all }}$, but fewer morphisms:

$$
\operatorname{Hom}_{\mathrm{HMF}}(M, N):=\operatorname{Hom}_{\mathrm{MF}}(M, N) /\{\text { null-homotopic morphisms\}. }
$$

Choose bases in $M^{0}$ and $M^{1}$ and write $d$ as a matrix $D$. Differentiating the equation $D^{2}=w$ with respect to $x_{i}$ we get

$$
D\left(\partial_{i} D\right)+\left(\partial_{i} D\right) D=\partial_{i} w .
$$

Therefore, the multiplication by $\partial_{i} w$ endomorphism of $M$ is homotopic to 0 , and we obtain 
Proposition 5. The action of $R$ on $\operatorname{Hom}_{\mathrm{HMF}}(M, N)$ factors through the action of the Jacobian algebra $R_{w}$.

Let $\mathrm{HMF}_{w}^{\prime}$ be the category with objects finite rank matrix factorizations and morphisms homomorphisms of factorizations modulo those homotopic to 0 . This is a full subcategory in $\mathrm{HMF}_{w}^{\text {all }}$.

The free $R$-module $\operatorname{Hom}_{R}(M, N)$ is a 2-complex

$$
\operatorname{Hom}_{R}^{0}(M, N) \stackrel{d}{\longrightarrow} \operatorname{Hom}_{R}^{1}(M, N) \stackrel{d}{\longrightarrow} \operatorname{Hom}^{0}(M, N),
$$

where

$$
\begin{aligned}
& \operatorname{Hom}_{R}^{0}(M, N)=\operatorname{Hom}_{R}\left(M^{0}, N^{0}\right) \oplus \operatorname{Hom}_{R}\left(M^{1}, N^{1}\right), \\
& \operatorname{Hom}_{R}^{1}(M, N)=\operatorname{Hom}_{R}\left(M^{0}, N^{1}\right) \oplus \operatorname{Hom}_{R}\left(M^{1}, N^{0}\right),
\end{aligned}
$$

and

$$
(d f) m=d_{N}(f(m))+(-1)^{i} f\left(d_{M}(m)\right) \quad \text { for } f \in \operatorname{Hom}_{R}^{i}(M, N) .
$$

Denote the cohomology of this 2-complex by

$$
\operatorname{Ext}(M, N)=\operatorname{Ext}^{0}(M, N) \oplus \operatorname{Ext}^{1}(M, N) .
$$

It is clear from the definitions that

$$
\begin{aligned}
& \operatorname{Ext}^{0}(M, N) \cong \operatorname{Hom}_{\operatorname{HMF}}(M, N), \\
& \operatorname{Ext}^{1}(M, N) \cong \operatorname{Hom}_{\text {HMF }}(M, N\langle 1\rangle) .
\end{aligned}
$$

Proposition 6. $\operatorname{Ext}(M, N)$ is a finite-dimensional $R_{w}$-module if $M$ and $N$ are finite rank factorizations.

Proof. We need to show that $\operatorname{Hom}_{\mathrm{HMF}}(M, N)$ is finite-dimensional. The latter is an $R$-module quotient of $\operatorname{Hom}_{\mathrm{MF}}(M, N)$, which is a submodule of the free finite rank $R$-module $\operatorname{Hom}_{R}(M, N)$. Since $R$ is Noetherian, subquotients of finitely generated $R$-modules are finitely generated. The action of $R$ on $\operatorname{Hom}_{\mathrm{HMF}}(M, N)$ factors through the action of the Jacobian ring $R_{w}$, by Proposition 5 . Therefore, $\operatorname{Hom}_{\mathrm{HMF}}(M, N)$ is finite-dimensional, being finitely generated over a finite-dimensional algebra.

REMARK. This proposition and many that follow fail to hold if $w \in R$ is not a potential. However, $(R, w)$-factorizations for such degenerate $w$ will make important intermediate appearances, as our main examples come from tensor products of factorizations with degenerate $w$ (when $R /(w)$ does not have an isolated singularity at 0 ).

Definition 4. A polynomial $p \in \mathfrak{m}^{2}$ is called a degenerate potential if the Jacobian algebra $R_{w}$ is infinite-dimensional.

Any polynomial in $\mathfrak{m}^{2}$ is either a potential or a degenerate potential (but never both). Unless specified otherwise, $w$ denotes a potential. 
The Tyurina algebra. The Tyurina algebra $R_{w}^{T}$ is defined as the quotient of the Jacobian algebra $R_{w}$ by the ideal generated by $w$. Multiplication by $w$ is homotopic to 0 in $\operatorname{Hom}_{\mathrm{MF}}(M, M)$, for any factorization $M$, and therefore $\operatorname{Hom}_{\mathrm{HMF}}(M, N)$ and $\operatorname{Ext}(M, N)$ are modules over the Tyurina algebra (this is a slight extension of Proposition 5).

Cohomology of factorizations. The quotient $M / \mathfrak{m} M$ is a 2-complex of vector spaces (the square of the differential is 0 since $w \in \mathfrak{m}$ ):

$$
M^{0} / \mathfrak{m} M^{0} \stackrel{d}{\longrightarrow} M^{1} / \mathfrak{m} M^{1} \stackrel{d}{\longrightarrow} M^{0} / \mathfrak{m} M^{0} .
$$

Denote the cohomology of this 2-complex by $H(M)$ and call it the cohomology of $M$. Note that $H(M)$ is $\mathbb{Z}_{2}$-graded, $H(M)=H^{0}(M) \oplus H^{1}(M)$. Cohomology of factorizations is a functor from $\mathrm{MF}_{w}^{\text {all }}$ and $\mathrm{HMF}_{w}^{\text {all }}$ to the category of $\mathbb{Z}_{2}$-graded $\mathbb{Q}$-vector spaces and grading-preserving linear maps.

Proposition 7. The following conditions on $M \in \mathrm{MF}_{w}^{\text {all }}$ are equivalent:

(i) $H(M)=0$.

(ii) $H^{0}(M)=0$.

(iii) $H^{1}(M)=0$.

(iv) $M$ is isomorphic to the zero factorization in the category $\mathrm{HMF}_{w}^{\text {all }}$.

(v) $M$ is isomorphic in $\mathrm{MF}_{w}^{\text {all }}$ to the (possibly infinite) direct sum of

$$
R \stackrel{1}{\longrightarrow} R \stackrel{w}{\longrightarrow} R
$$

and

$$
R \stackrel{w}{\longrightarrow} R \stackrel{1}{\longrightarrow} R \text {. }
$$

Proof. The implications (v) $\Rightarrow($ iv $) \Rightarrow($ i $) \Rightarrow$ (ii) and (i) $\Rightarrow$ (iii) are obvious. It suffices to establish (ii) $\Rightarrow(\mathrm{v})$. Choose $R$-module bases in $M^{0}$ and $M^{1}$ and write factorization maps in matrix form. If one of the entries does not lie in the maximal ideal $\mathfrak{m} \subset R$, this entry is invertible, and a change of bases makes the matrices block-diagonal with two blocks, one of which is either $(1, w)$ or $(w, 1)$. Therefore, $M$ is isomorphic to the direct sum of some factorization $N$ and either (6) or (7). Applying Zorn's lemma, we conclude that any factorization $M$ decomposes into a direct sum $M \cong M_{e s} \oplus M_{c}$ where $M_{c}$ is a direct sum of factorizations (6) or (7) and $M_{e s}$ does not have any invertible elements in its matrix presentation (equivalently, $M_{e s}$ does not contain any summands isomorphic to (6) or (7)). Since $M_{e s}^{0} \cong V \otimes_{\mathbb{Q}} R$ as $R$-modules, for some vector space $V$, we have

$$
H^{0}(M) \cong H^{0}\left(M_{e s}\right) \cong V .
$$

Therefore, if $M$ satisfies (ii), then $V=0$ and $M_{e s}=0$. The proposition follows. 
A factorization is called contractible if it satisfies one of the five equivalent conditions in Proposition 7.

Define the dimension $\operatorname{dim}(M)$ of the factorization $M$ as the dimension of the $\mathbb{Q}$-vector space $H(M)$. We have $\operatorname{dim}(M) \leq 2 \operatorname{rk}(M)$. If $M$ is a finite rank factorization, equality holds iff $M$ does not contain contractible summands.

EXAMPLE. A factorization of rank 1 has the form

$$
R \stackrel{a_{0}}{\longrightarrow} R \stackrel{a_{1}}{\longrightarrow} R
$$

for $a_{0}, a_{1} \in R$ such that $w=a_{0} a_{1}$. Denote this factorization by $N$. Then

$$
\operatorname{Hom}_{\mathrm{MF}}(N, N) \cong R \text { and } \operatorname{Hom}_{\mathrm{HMF}}(N, N) \cong R /\left(a_{0}, a_{1}\right),
$$

the quotient of $R$ by the ideal generated by $a_{0}$ and $a_{1}$. The factorization $N$ is contractible iff either $a_{0}$ or $a_{1}$ is invertible (does not lie in $\mathfrak{m}$ ). It is nontrivial when both $a_{0}$ and $a_{1}$ belong to the maximal ideal (then $H(M) \cong \mathbb{Q} \oplus \mathbb{Q}$ ). This is only possible if $k$, the number of variables in the set $x$, is at most 2 (since we need $w=a_{0} a_{1}$ to be nondegenerate).

Proposition 8. The following properties of a morphism $f: M \rightarrow N$ of factorizations are equivalent:

(i) $f$ is an isomorphism in the homotopy category of factorizations.

(ii) $f$ induces an isomorphism between the cohomologies of $M$ and $N$.

Proof. The implication (i) $\Rightarrow$ (ii) is obvious. To prove (ii) $\Rightarrow(\mathrm{i})$, choose decompositions $M \cong M_{e s} \oplus M_{c}$ and $N \cong N_{e s} \oplus N_{c}$. The composition

$$
f_{\text {es }}: M_{e s} \stackrel{i}{\longrightarrow} M \stackrel{f}{\longrightarrow} N \stackrel{p}{\longrightarrow} N_{e s},
$$

where $i$ and $p$ are the obvious inclusion and projection, induces an isomorphism $H\left(f_{e s}\right): H\left(M_{e s}\right) \cong H\left(N_{e s}\right)$. Given two free $R$-modules $L_{1}$ and $L_{2}$, an $R$-module map $L_{1} \rightarrow L_{2}$ which induces an isomorphism of quotients $L_{1} / \mathfrak{m} L_{1} \cong L_{2} / \mathfrak{m} L_{2}$ is an isomorphism of $R$-modules. Therefore, $f_{e s}: M_{e s} \rightarrow$ $N_{e s}$ is an isomorphism of $R$-modules, and $f$ is an isomorphism in the homotopy category of factorizations.

REMARK. We see from this proposition that, although $\mathrm{HMF}_{w}^{\text {all }}$ was defined as a "homotopy" category, it also has the flavor of a "derived" category: in the latter, a morphism which induces an isomorphism in cohomology is an isomorphism. For an explanation and generalizations of this phenomenon see Buchweitz [B] and Orlov [O].

The proof of Proposition 8 implies

Corollary 3. A decomposition $M \cong M_{e s} \oplus M_{c}$ of $M$ into a factorization without contractible summands and a contractible factorization is unique up to isomorphism.

We call $M_{e s}$ the essential summand of $M$. 
Corollary 4. Any factorization $M$ with finite-dimensional cohomology is a direct sum of a finite rank factorization and a contractible factorization.

Let $\mathrm{MF}_{w}$ be the category whose objects are factorizations with finitedimensional cohomology and morphisms are homomorphisms of factorizations. Let $\mathrm{HMF}_{w}$ be the quotient of $\mathrm{MF}_{w}$ by the ideal of null-homotopic morphisms. Any finite rank factorization has finite-dimensional cohomology, therefore, we have (full and faithful) inclusions of categories $\mathrm{MF}_{w}^{\prime} \subset \mathrm{MF}_{w}$ and $\mathrm{HMF}_{w}^{\prime} \subset \mathrm{HMF}_{w}$. Corollaries 3 and 4 imply

COROllary 5. The inclusion $\mathrm{HMF}_{w}^{\prime} \subset \mathrm{HMF}_{w}$ is an equivalence of categories.

Although we will be doing most of our work in the categories $\mathrm{HMF}_{w}$ for various $w$, the other five categories provide a useful supporting framework. To reduce the confusion of six different categories, we arranged them into a table.

\begin{tabular}{|l|c|c|c|}
\hline & $\begin{array}{c}\text { all } \\
\text { factorizations }\end{array}$ & $\begin{array}{c}\text { finite } \\
\text { rank }\end{array}$ & $\begin{array}{c}\text { factorizations with } \\
\text { finite-dimensional } \\
\text { cohomology }\end{array}$ \\
\hline $\begin{array}{l}\text { all } \\
\text { homomorphisms }\end{array}$ & $\mathrm{MF}_{w}^{\text {all }}$ & $\mathrm{MF}_{w}^{\prime}$ & $\mathrm{MF}_{w}$ \\
\hline $\begin{array}{l}\text { modulo } \\
\text { homotopic to 0 }\end{array}$ & $\mathrm{HMF}_{w}^{\text {all }}$ & $\mathrm{HMF}_{w}^{\prime}$ & $\cong \mathrm{HMF}_{w}$ \\
\hline
\end{tabular}

The categories in the bottom row are triangulated, and the two rightmost categories in this row are equivalent. Even though most of the time our constructions start with finite rank factorizations, our functors take them to infinite rank factorizations, but with finite-dimensional cohomology. The latter factorizations lie in $\mathrm{HMF}_{w}$ (they can be reduced to finite rank factorizations, but this operation, sometimes necessary on concrete factorizations, is awkward from the categorical viewpoint). At the same time, the collection of categories $\mathrm{HMF}_{w}$ over various $w$ is closed under all functors that are essential in our work, and is a natural and thrifty choice.

Dualities. The free $R$-module $M^{*}=\operatorname{Hom}_{R}(M, R)$ admits a factorization

$$
\left(M^{0}\right)^{*} \stackrel{\left(d^{1}\right)^{*}}{\longrightarrow}\left(M^{1}\right)^{*} \stackrel{\left(d^{0}\right)^{*}}{\longrightarrow}\left(M^{0}\right)^{*} .
$$

An inclusion of factorizations $M \subset M^{* *}$ is an isomorphism if $M$ has finite rank, and an isomorphism in the homotopy category if $M$ has finitedimensional cohomology. Thus, $M \rightarrow M^{*}$ is a contravariant equivalence in the categories $\mathrm{MF}_{w}^{\prime}, \mathrm{HMF}_{w}^{\prime}$, and $\mathrm{HMF}_{w}$. 
Assume that $M$ has finite rank, and choose $R$-module bases in $M^{0}, M^{1}$. The factorization is described by two matrices $\left(D_{0}, D_{1}\right)$. The dual factorization $M^{*}$ is described by the transposed matrices $\left(D_{1}^{t}, D_{0}^{t}\right)$.

For $M \in \mathrm{MF}_{w}^{\text {all }}$ denote by $M_{-}$the factorization

$$
M^{0} \stackrel{-d^{0}}{\longrightarrow} M^{1} \stackrel{d^{1}}{\longrightarrow} M^{0} \text {. }
$$

This assignment extends to an equivalence of categories of factorizations with potentials $w$ and $-w$.

Let $M_{\bullet}=\left(M^{*}\right)_{-}$. This assignment is a contravariant functor between categories of factorizations with potentials $w$ and $-w$. Observe that

$$
\{\mathbf{a}, \mathbf{b}\}_{\bullet} \cong\{-\mathbf{b}, \mathbf{a}\} \text {. }
$$

Excluding a variable. Let $R=\mathbb{Q}\left[\left[x_{1}, \ldots, x_{m}\right]\right]$ and $R^{\prime}=\mathbb{Q}\left[\left[x_{2}, \ldots, x_{m}\right]\right]$ $\subset R$. Suppose that $w \in R^{\prime}$ is a potential, and $w=\mathbf{a b}$ for a pair $\{\mathbf{a}, \mathbf{b}\}$ as in Section 2, where $a_{i}, b_{i} \in R$. Suppose $b_{i}-x_{1} \in R^{\prime}$ for some $i$. Let $c=b_{i}-x_{1}$, and $\mathbf{a}^{i}, \mathbf{b}^{i}$ be the sequences obtained from $\mathbf{a}$ and $\mathbf{b}$ by omitting $a_{i}$ and $b_{i}$. Let $\psi: R \rightarrow R^{\prime}$ be the homomorphism $\psi\left(x_{j}\right)=x_{j}$ for $j \neq 1$, and $\psi\left(x_{1}\right)=-c$ (so that $\left.\psi\left(b_{i}\right)=0\right)$. Note that $\psi(w)=w$.

Let $\psi\left(\mathbf{a}^{i}\right), \psi\left(\mathbf{b}^{i}\right)$ be the sequences obtained by applying $\psi$ to every entry of $\mathbf{a}^{i}$ and $\mathbf{b}^{i}$. Then $\left\{\psi\left(\mathbf{a}^{i}\right), \psi\left(\mathbf{b}^{i}\right)\right\}$ is an $\left(R^{\prime}, w\right)$-factorization. By treating $R$ as an $R^{\prime}$-module, we can view $\{\mathbf{a}, \mathbf{b}\}$ as an $\left(R^{\prime}, w\right)$-factorization (of infinite rank). Let

$$
f:\{\mathbf{a}, \mathbf{b}\} \rightarrow\left\{\psi\left(\mathbf{a}^{i}\right), \psi\left(\mathbf{b}^{i}\right)\right\}
$$

be the following homomorphism of $\left(R^{\prime}, w\right)$-factorizations:

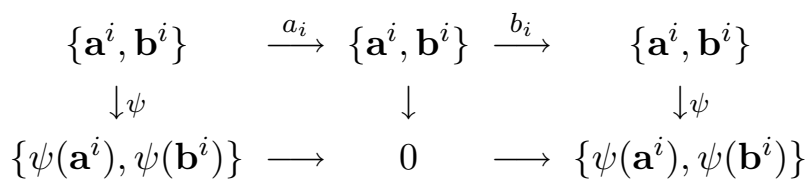

The top line is the factorization $\{\mathbf{a}, \mathbf{b}\}$ written as the total factorization of a bifactorization.

Proposition 9. $f$ is an isomorphism in the homotopy category of $\left(R^{\prime}, w\right)$-factorizations.

Proof. It would suffice to show that $f$ induces an isomorphism on cohomology. Multiplication by $b_{i}$ is an injective endomorphism of the $R^{\prime}$-module $\left\{\mathbf{a}^{i}, \mathbf{b}^{i}\right\}$. Decompose

$$
\left\{\mathbf{a}^{i}, \mathbf{b}^{i}\right\} \cong b_{i}\left\{\mathbf{a}^{i}, \mathbf{b}^{i}\right\} \oplus M,
$$

where the decomposition is that of $R^{\prime}$-modules, and $M$ consists of vectors with all coordinates in $R^{\prime}$ in the standard $R$-module basis of $\left\{\mathbf{a}^{i}, \mathbf{b}^{i}\right\}$. The $R^{\prime}$-subfactorization

$$
b_{i}\left\{\mathbf{a}^{i}, \mathbf{b}^{i}\right\} \stackrel{a_{i}}{\longrightarrow}\left\{\mathbf{a}^{i}, \mathbf{b}^{i}\right\} \stackrel{b_{i}}{\longrightarrow} b_{i}\left\{\mathbf{a}^{i}, \mathbf{b}^{i}\right\}
$$


of $\{\mathbf{a}, \mathbf{b}\}$ is contractible, while $f(M)=\left\{\psi\left(\mathbf{a}^{i}\right), \psi\left(\mathbf{b}^{i}\right)\right\}$ as $R^{\prime}$-modules. It follows immediately that $f$ induces an isomorphism on cohomology.

Let $R=\mathbb{Q}\left[\left[x_{1}, \ldots, x_{k+m}\right]\right]$ and $R^{\prime}=\mathbb{Q}\left[\left[x_{k+1}, \ldots, x_{k+m}\right]\right] \subset R$. Suppose that $w \in R^{\prime}$ is a potential, and $w=\mathbf{a b}$ for a pair $\{\mathbf{a}, \mathbf{b}\}$ with $a_{j}, b_{j} \in R$. Assume that $b_{1}-x_{1}$ is a polynomial in $x_{2}, \ldots, x_{k+m}$, and, more generally, $b_{j}-x_{j}$, for each $j$ between 1 and $k$, is a polynomial in $x_{j+1}, \ldots, x_{k+m}$, modulo the ideal generated by $b_{1}, b_{2}, \ldots, b_{j-1}$. Let $\psi$ be the homomorphism $R \rightarrow R^{\prime}$ uniquely determined by the conditions $\psi\left(x_{j}\right)=x_{j}$ for $j>k$, and $\psi\left(b_{j}\right)=0$ for $j \leq k$. Let $\mathbf{a}_{k}, \mathbf{b}_{k}$ be the sequences obtained from $\mathbf{a}, \mathbf{b}$ by omitting $a_{j}, b_{j}$ for all $j \leq k$. Let $\psi\left(\mathbf{a}_{k}\right), \psi\left(\mathbf{b}_{k}\right)$ be the sequences obtained by applying $\psi$ to every entry of $\mathbf{a}_{k}$ and $\mathbf{b}_{k}$. Then $\left\{\psi\left(\mathbf{a}_{k}\right), \psi\left(\mathbf{b}_{k}\right)\right\}$ is an $\left(R^{\prime}, w\right)$-factorization. Treating $R$ as an $R^{\prime}$-module, we view $\{\mathbf{a}, \mathbf{b}\}$ as an $\left(R^{\prime}, w\right)$-factorization (of infinite rank).

Proposition 10. $\{\mathbf{a}, \mathbf{b}\}$ and $\left\{\psi\left(\mathbf{a}_{k}\right), \psi\left(\mathbf{b}_{k}\right)\right\}$ are isomorphic in the homotopy category of $\left(R^{\prime}, w\right)$-factorizations.

Proof. The proof of the previous proposition generalizes to this setup without difficulty.

Frobenius structure. The following duality theorem was proved by Ragnar-Olaf Buchweitz [B] in much greater generality.

TheOREM 1. Suppose that $w$ is a potential in an even number of variables, $w=w\left(x_{1}, \ldots, x_{2 k}\right)$. There exists a collection of trace maps

$$
\operatorname{Tr}_{M}: \operatorname{Hom}_{\mathrm{HMF}}(M, M) \rightarrow \mathbb{Q},
$$

indexed by objects of $\mathrm{HMF}_{w}$, such that for any $M, N \in \mathrm{Ob}\left(\mathrm{HMF}_{w}\right)$ the composition

$$
\operatorname{Hom}_{\mathrm{HMF}}(M, N) \otimes \operatorname{Hom}_{\mathrm{HMF}}(N, M) \rightarrow \operatorname{Hom}_{\mathrm{HMF}}(M, M) \stackrel{\operatorname{Tr}_{M}}{\longrightarrow} \mathbb{Q}
$$

is a nondegenerate bilinear pairing.

Proof. See Theorem 7.7.5, Proposition 10.1.5, Example 10.1.6, and Corollary 10.3.3 in [B].

Although Theorem 1 is not used explicitly in this paper, the Frobenius structure of the categories $\mathrm{HMF}_{w}$ is implicit in several of our constructions and proofs. We expect that Theorem 1 will become indispensable in further investigations of the interplay between matrix factorizations and link homology.

\section{Factorizations as functors}

Internal tensor product. Let $M \in \mathrm{MF}_{w}^{\text {all }}$ and $N \in \mathrm{MF}_{-w}^{\text {all }}$. The tensor product $M \otimes_{R} N$ is a 2-complex 


$$
\left(M \otimes_{R} N\right)^{0} \stackrel{d}{\longrightarrow}\left(M \otimes_{R} N\right)^{1} \stackrel{d}{\longrightarrow}\left(M \otimes_{R} N\right)^{0},
$$

where

$$
\left(M \otimes_{R} N\right)^{j}=\bigoplus_{i \in\{0,1\}} M^{i} \otimes_{R} N^{j-i}
$$

and

$$
d(m \otimes n)=d_{M}(m) \otimes n+(-1)^{i} m \otimes d_{N}(n), \quad m \in M^{i} .
$$

Proposition 11. $M \otimes_{R} N$ has finite-dimensional cohomology if $M$ and $N$ are factorizations with finite-dimensional cohomology.

Proof. This is a special case of Proposition 13 below.

Proposition 12. If $M$ has finite rank, there is a natural isomorphism of 2-complexes

$$
\operatorname{Hom}_{R}(M, N) \cong N \otimes_{R} M_{\bullet}
$$

Proof. The usual isomorphism of $R$-modules on the left and right hand sides of (8) intertwines the differentials in these 2-complexes.

Corollary 6. If $M$ has finite-dimensional cohomology, there is a natural isomorphism of cohomology groups

$$
\operatorname{Ext}(M, N) \cong \mathrm{H}\left(N \otimes_{R} M_{\bullet}\right),
$$

and (in the homotopy category) of 2-complexes

$$
\operatorname{Hom}_{R}(M, N) \cong N \otimes_{R} M_{\bullet}
$$

The internal tensor product $M \otimes N$ will be depicted by gluing the diagram's arcs (see Figure 13 right).
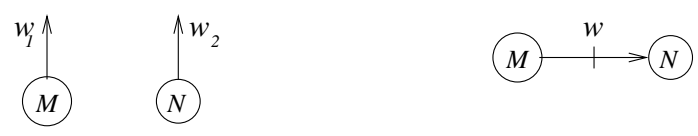

Fig. 13. External and internal tensor products

Tensor product for sums of potentials. To add dynamics to the world of matrix factorizations we need a large supply of functors between categories of factorizations over various isolated singularities $w$. The tensor product (over $\mathbb{Q}[[y]]$ ) with a matrix factorization $M \in \mathrm{MF}_{w_{1}(x)-w_{2}(y)}$ is a functor from $\mathrm{MF}_{w_{2}(y)}^{\text {all }}$ to $\mathrm{MF}_{w_{1}(x)}^{\text {all }}$. A slightly more general construction requires three potentials $w_{1}, w_{2}, w_{3}$ and two factorizations $M \in \mathrm{MF}_{w_{1}(x)-w_{2}(y)}^{\text {all }}, N \in$ $\mathrm{MF}_{w_{2}(y)-w_{3}(z)}^{\text {all }}$. We define their tensor product $M \otimes_{y} N$ by

$$
\left(M \otimes_{y} N\right)^{i}=\bigoplus_{j \in\{0,1\}}\left(M^{j} \otimes_{y} N^{i-j}\right)
$$


and

$$
d(m \otimes n)=d_{M}(m) \otimes n+(-1)^{i} m \otimes d_{N}(n) \quad \text { if } m \in M^{i} .
$$

Here $M^{j} \otimes_{y} N^{i-j}$ denotes the completed tensor product $M^{j} \widehat{\otimes}_{Q[[y]]} N^{i-j}$ (in the sense that the ring $\mathbb{Q}[[x, y, z]]$ of power series in the variables $x, y, z$ is a completion of $\left.\mathbb{Q}[[x, y]] \otimes_{\mathbb{Q}[[y]]} \mathbb{Q}[[y, z]]\right)$.

If $M$ or $N$ is contractible, so is their tensor product. The tensor product can be viewed as a bifunctor

$$
\begin{aligned}
\mathrm{MF}_{w_{1}(x)-w_{2}(y)}^{\text {all }} \times \mathrm{MF}_{w_{2}(y)-w_{3}(z)}^{\text {all }} & \rightarrow \mathrm{MF}_{w_{1}(x)-w_{3}(z)}^{\text {all }}, \\
\mathrm{HMF}_{w_{1}(x)-w_{2}(y)}^{\text {all }} \times \mathrm{HMF}_{w_{2}(y)-w_{3}(z)}^{\text {all }} & \rightarrow \mathrm{HMF}_{w_{1}(x)-w_{3}(z)}^{\text {all }} .
\end{aligned}
$$

The tensor product does not preserve the finite rank property (since $\mathbb{Q}[[x, y, z]]$ has infinite rank as a $\mathbb{Q}[[x, z]]$-module if the set of variables $y$ is nonempty). However, we have

Proposition 13. If $M$ and $N$ have finite-dimensional cohomology, so does their tensor product.

This proposition can be restated by saying that tensor product restricts to bifunctors

$$
\begin{aligned}
\mathrm{MF}_{w_{1}(x)-w_{2}(y)} \times \mathrm{MF}_{w_{2}(y)-w_{3}(z)} & \rightarrow \mathrm{MF}_{w_{1}(x)-w_{3}(z)}, \\
\mathrm{HMF}_{w_{1}(x)-w_{2}(y)} \times \mathrm{HMF}_{w_{2}(y)-w_{3}(z)} & \rightarrow \mathrm{HMF}_{w_{1}(x)-w_{3}(z)} .
\end{aligned}
$$

Proof. It suffices to show that $T=M \otimes_{y} N$ has finite-dimensional cohomology if $M$ and $N$ have finite rank. This cohomology $H(T)$ is a module over the Jacobian algebra $R_{f_{2}(y)}$, where $R=\mathbb{Q}[[y]]$, and a subquotient of the finitely generated free $\mathbb{Q}[[y]]$-module $T /(x, z) T$. Thus, $H(T)$ is a finitely generated $R_{f_{2}(y)}$-module, and necessarily has finite dimension.

The tensor product of factorizations $M$ and $N$ will be depicted by joining the matching ends of their diagrams and placing a mark at the joint, as Figure 14 illustrates. Sometimes we will write $M$ as $M_{y}^{x}$ and $N$ as $N_{z}^{y}$, and their tensor product as $M_{y}^{x} N_{z}^{y}$.

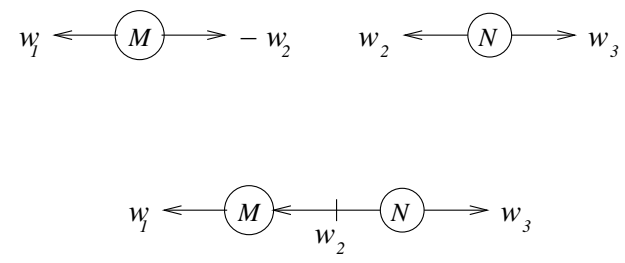

Fig. 14. Graphical presentation of the tensor product over $\mathbb{Q}[[y]]$

If the summands $w_{1}(x)$ and $w_{3}(z)$ of the potentials for $M$ and $N$ are themselves sums of potentials, and we want to emphasize these decompositions, we will denote the tensor product $M \otimes_{y} N$ as in Figure 15. 

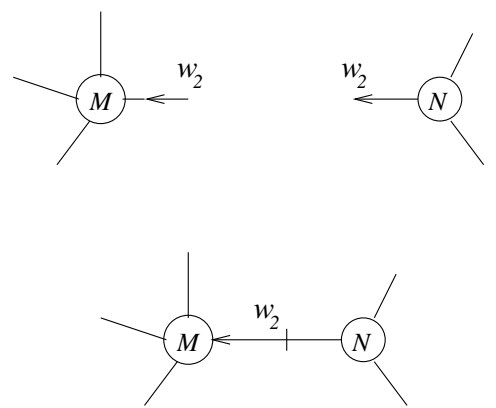

Fig. 15. Tensor product over $\mathbb{Q}[[y]]$ when $w_{1}$ and $w_{3}$ are sums

REMARK. Matrix factorizations over exterior sums of potentials are studied in $[\mathrm{EP}],[\mathrm{HP}],[\mathrm{P}]$, and $[\mathrm{Y} 2]$.

If a factorization $M$ has potential $w(x)-w(y)+w_{1}(z)$, then the quotient $M /(x-y) M$ is a module over the ring $\mathbb{Q}[[x, y, z]] /(x-y)$. We treat $M /(x-y) M$ as a factorization, necessarily of infinite rank, with potential $w_{1}(z)$ over the ring $\mathbb{Q}[[z]]$ (were we to consider $M /(x-y) M$ over the larger ring $\mathbb{Q}[[x, z]]$, the potential would have been degenerate).

Proposition 14. If a factorization $M$ with potential $w(x)-w(y)+w_{1}(z)$ has finite-dimensional cohomology, so does $M /(x-y) M$.

Proof. Similar to the one of Proposition 13.

We depict the quotient factorization by joining the $x$ and $y$ legs of $M$, and placing a mark where the legs were joined (see Figure 16).
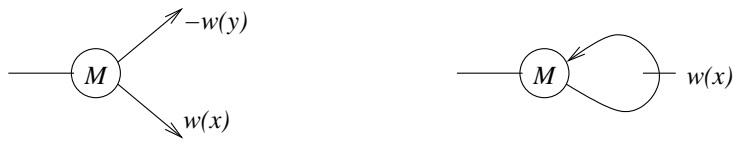

Fig. 16. $M$ and its quotient $M /(x-y) M$

A closed diagram of factorizations (as in Figure 17) gives rise to a tensor product, the latter a two-periodic complex of modules over a suitable power series ring. Each oriented edge has a finite set of variables and a potential assigned to it, $d^{2}=0$ since the potentials cancel. If each factorization in the diagram has finite-dimensional cohomology, the complex will have finitedimensional cohomology as well. The network does not even have to be planar, and does not need to be embedded anywhere. In our paper, however, all such diagrams are going to be planar.

External tensor product. When the intermediate set of variables is empty, the (completed) tensor product is over $\mathbb{Q}$, and we call it the external tensor product $M \otimes_{\mathbb{Q}} N$. This operation was investigated by Yoshino [Y2]. 


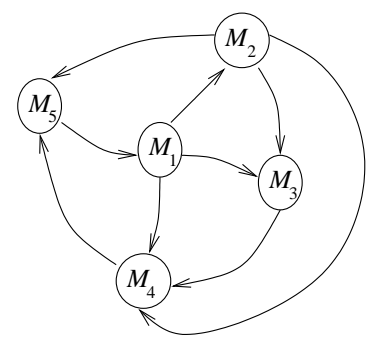

Fig. 17. A closed diagram of factorizations; $d^{2}=0$ in the tensor product, marks on the edges are omitted.

The disjoint union of two diagrams denotes the external tensor product of corresponding factorizations (see Figure 13 left).

Hiding minus signs in tensor products. Suppose $I$ is a finite set and $M_{a}$, $a \in I$, is a collection of factorizations (possibly with degenerate potentials). To define the differential in the tensor product $\bigotimes_{a \in I} M_{a}$ in a manifestly intrinsic way, consider the Clifford ring $C l(I)$ of the set $I$. It has generators $a \in I$ and relations

$$
a^{2}=1, \quad a b+b a=0, \quad a \neq b .
$$

As an abelian group, $C l(I)$ has rank $2^{|I|}$, where $|I|$ is the cardinality of $I$, and breaks down into a direct sum

$$
C l(I)=\bigoplus_{J \subset I} \mathbb{Z}_{J}
$$

$\mathbb{Z}_{J}$ has as generators all ways to order elements of the set $J$, and relations

$$
a \ldots b c \ldots e+a \ldots c b \ldots e=0
$$

for all orderings $a \ldots b c \ldots e$ of $J$. The group $\mathbb{Z}_{J}$ is isomorphic to $\mathbb{Z}$, but there is no canonical isomorphism.

$r_{a}^{2}=1$ where $r_{a}$ is the right multiplication by $a$ endomorphism of $C l(I)$. For each $J \subset I$ which does not contain $a$ we have a 2-periodic sequence

$$
\mathbb{Z}_{J} \stackrel{r_{a}}{\longrightarrow} \mathbb{Z}_{J \sqcup\{a\}} \stackrel{r_{a}}{\longrightarrow} \mathbb{Z}_{J}
$$

Now define the tensor product factorization of $M_{a}$ 's as the sum, over all $J \subset I$, of

$$
\left(\bigotimes_{a \in J} M_{a}^{1}\right) \otimes\left(\bigotimes_{b \in I \backslash J} M_{b}^{0}\right) \otimes_{\mathbb{Z}} \mathbb{Z}_{J}
$$

with the differential

$$
d=\sum_{a \in I} d_{a} \otimes r_{a}
$$

where $d_{a}$ is the differential in $M_{a}$. We denote this tensor product by $\bigotimes_{a \in I} M_{a}$.

When forming tensor products of factorizations, we will use this trick, and would need to assign labels to each term in a tensor product. If a label 
$a$ is assigned to a factorization $M$, we write $M$ as

$$
M^{0}(\emptyset) \rightarrow M^{1}(a) \rightarrow M^{0}(\emptyset) .
$$

Factorization of the identity functor. Start with the one-variable potential $w(x)=x^{n+1}$ and consider the potential $w(x)-w(y)$ in two variables. Let

$$
\pi_{x y}=\frac{w(x)-w(y)}{x-y}=x^{n}+x^{n-1} y+\cdots+y^{n} .
$$

Denote by $L_{y}^{x}$ the factorization

$$
R \stackrel{\pi_{x y}}{\longrightarrow} R \stackrel{x-y}{\longrightarrow} R
$$

where $R=\mathbb{Q}[[x, y]]$. We have

$$
\begin{aligned}
\operatorname{Hom}_{\text {HMF }}\left(L_{y}^{x}, L_{y}^{x}\right) & \cong \mathbb{Q}[x] /\left(x^{n}\right), \\
\operatorname{Hom}_{\mathrm{HMF}}\left(L_{y}^{x}, L_{y}^{x}\langle 1\rangle\right) & \cong 0 .
\end{aligned}
$$

Indeed, $\operatorname{Hom}_{R}\left(L_{y}^{x}, L_{y}^{x}\right)$ is isomorphic to the Koszul complex of the sequence $\left(x-y, \pi_{x y}\right)$, with the grading collapsed from $\mathbb{Z}$ to $\mathbb{Z}_{2}$. Regularity of this sequence implies the formulas (of course, it is easy to check the above two formulas directly; for instance, the second formula follows since $x-y$ and $\pi_{x y}$ are relatively prime). If we assign label $(a)$ to $L_{y}^{x}$, we can write this factorization as

$$
R(\emptyset) \stackrel{\pi_{x y}}{\longrightarrow} R(a) \stackrel{x-y}{\longrightarrow} R(\emptyset) .
$$

Here $R(\emptyset), R(a)$ are free $R$-modules of rank 1 with basis vectors $1(\emptyset), 1(a)$. The differential takes $1(\emptyset)$ to $\pi_{x y} \cdot 1(a)$, and $1(a)$ to $(x-y) \cdot 1(\emptyset)$.

We depict $L_{y}^{x}$ by an arc oriented from $y$ to $x$ (see Figure 18). If the potential assigned to an endpoint of a diagram has the form $x^{n+1}$ for some variable $x$, we just write the variable at the endpoint, rather than the potential.

$$
L_{y}^{x} \quad x \longleftarrow y
$$

Fig. 18. Arc with endpoints $x$ and $y$

Proposition 15. There is a natural isomorphism

$$
L_{y}^{x} M_{z}^{y} \cong M_{z}^{x}
$$

where $w_{1}(z)$ is any potential in variables $z$, and $M_{z}^{y}$ any factorization over $y^{n+1}-w_{1}(z)$.

(Notation $L_{y}^{x} M_{z}^{y}$ was explained several pages earlier.)

Proof. Assign labels $a, b, c$ to factorizations $L_{y}^{x}, M_{z}^{y}$, and $M_{z}^{x}$, respectively. The map of $w(x)-w_{1}(z)$ factorizations

$$
\tau_{1}: L_{y}^{x} \otimes_{y} M_{z}^{y} \rightarrow M_{z}^{x}
$$


defined by taking $R(a) \otimes_{y} M_{z}^{y}$ to 0 and $R(\emptyset) \otimes_{y} M_{z}^{y} \cong \mathbb{Q}[[x, y]] \otimes_{y} M_{z}^{y}$ onto $M_{z}^{x}$ by adding the relation $x=y$ induces an isomorphism on cohomologies of the two factorizations.

This isomorphism is functorial in $M$ and implies

COROLlaRY 7. Tensor product with $L_{y}^{x}$ is an invertible functor from the homotopy category of matrix factorizations with potential $y^{n+1}-w_{1}(z)$ to the homotopy category of matrix factorizations with potential $x^{n+1}-w_{1}(z)$, for any potential $w_{1}(z)$, and is isomorphic to the substitution functor that relabels $y$ as $x$.

We could say informally that the tensor product with $L_{y}^{x}$ is the identity functor. We depict $L_{y}^{x} M_{z}^{y}$ by gluing their $y$-endpoints and placing a mark at the gluing point (see Figure 19 top). The proposition can be interpreted graphically as an isomorphism in Figure 19.

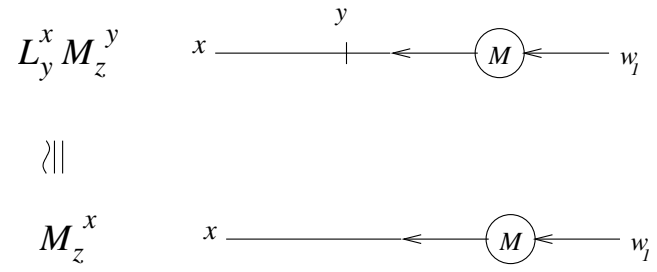

Fig. 19. Removing a mark

Proposition 16. The diagram in Figure 20 is commutative.

Proof. In other words, for two marks on different arcs, the order in which they are removed does not matter. The proof is a simple computation with the maps $\tau_{1}$.

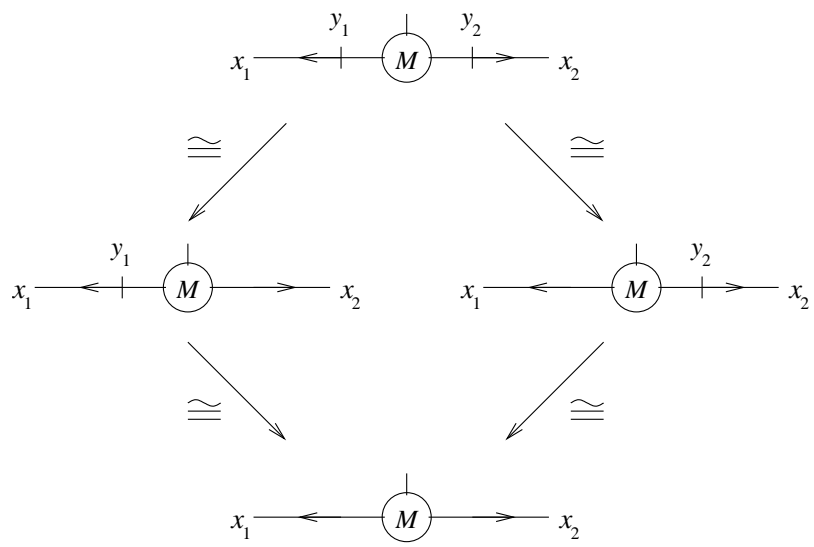

Fig. 20. Commutative diagram of mark removals 
Proposition 17. There is a natural isomorphism

$$
M_{x}^{z} L_{y}^{x} \cong M_{y}^{z}
$$

where $w_{1}(z)$ is any potential in the variables $z$, and $M_{z}^{x}$ any factorization over $w_{1}(z)-x^{n+1}$.

Proof. Assign labels $a, b, c$ to factorizations $M_{x}^{z}, L_{y}^{x}$, and $M_{z}^{y}$, respectively. The map of $w_{1}(z)-y^{n+1}$ factorizations

$$
\tau_{2}: M_{x}^{z} \otimes_{x} L_{y}^{x} \rightarrow M_{y}^{z}
$$

defined by taking $M_{x}^{z} \otimes_{y} R(b)$ to 0 and $M_{x}^{z} \otimes_{x} R(\emptyset) \cong M_{x}^{z} \otimes_{x} \mathbb{Q}[[x, y]]$ onto $M_{y}^{z}$ by adding the relation $x=y$ induces an isomorphism on cohomologies of the two factorizations.

The proposition can be interpreted graphically as an isomorphism in Figure 21.

$$
\begin{gathered}
M_{x}^{z} L_{y}^{x} \\
M_{y}^{z}
\end{gathered}
$$$$
w_{1}<M<\stackrel{x}{1}, y
$$

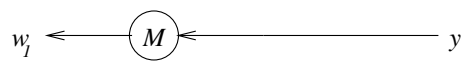

Fig. 21. Removing a mark

The isomorphism is functorial in $M$ and implies

COROLlaRY 8. Tensor product with $L_{y}^{x}$ is an invertible functor from the homotopy category of matrix factorizations with potential $w_{1}(z)-x^{n+1}$ to the homotopy category of matrix factorizations with potential $w_{1}(z)-y^{n+1}$, for any potential $w_{1}(z)$, and is isomorphic to the substitution functor that relabels $x$ as $y$.

Commutative diagram 20 admits three other versions, with reversed orientation in one or two of the $x_{1}, x_{2}$ legs of $M$ and mark removal using the morphism $\tau_{2}$ instead of $\tau_{1}$. Each of these three diagrams is commutative.

In Figure 22 we have two marks on an arc connecting the factorizations $N$ and $M$. We could remove the mark labeled $y$ using $\tau_{1}$, or we could remove the other mark (via $\tau_{2}$ ), and then relabel $y$ as $x$.

Proposition 18. The two resulting morphisms from the top to the bottom left factorizations in Figure 22 are equal.

Proof. Direct computation.

There are other versions of Figure 22, with the orientation of the marked arc reversed, and with $N$ and $M$ being just one factorization (so that the 


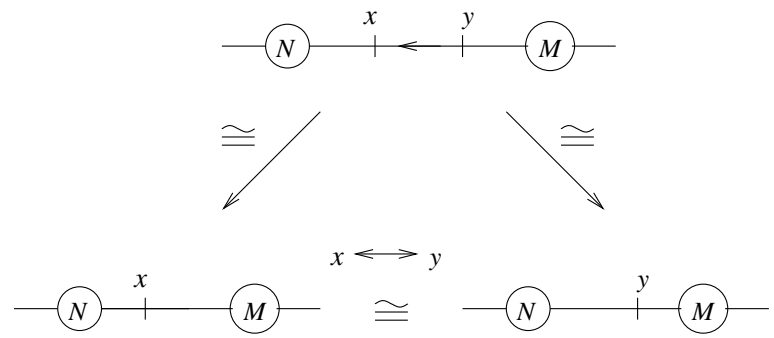

Fig. 22. Yet another commutative diagram

arc starts and ends in the same factorization). Commutativity holds in all of these cases.

We now specialize to the case when $M$ is itself an arc. The tensor product $L_{y}^{x} L_{z}^{y}$ is the factorization

$$
\left(\begin{array}{c}
R(\emptyset) \\
R(a b)
\end{array}\right) \stackrel{\left(\begin{array}{ll}
\pi_{x y} & y-z \\
\pi_{y z} & y-x
\end{array}\right)}{\longrightarrow}\left(\begin{array}{c}
R(a) \\
R(b)
\end{array}\right) \stackrel{\left(\begin{array}{cc}
x-y & y-z \\
\pi_{y z} & -\pi_{x y}
\end{array}\right)}{\longrightarrow}\left(\begin{array}{c}
R(\emptyset) \\
R(a b)
\end{array}\right)
$$

where $R=\mathbb{Q}[[x, y, z]]$, and we assigned labels $a, b$ to $L_{y}^{x}, L_{z}^{y}$, respectively. The minus sign in front of $\pi_{x y}$ in the right $2 \times 2$ matrix comes from the relation $b a=-a b$.

$L_{z}^{x}$ is given by

$$
R^{\prime}(\emptyset) \stackrel{\pi_{x z}}{\longrightarrow} R^{\prime}(c) \stackrel{x-z}{\longrightarrow} R^{\prime}(\emptyset)
$$

where $R^{\prime}=\mathbb{Q}[[x, z]]$ (and notice label $c$ ).

We can specialize maps $\tau_{1}, \tau_{2}$, introduced earlier, to this case.

The map $\tau_{1}: L_{y}^{x} L_{z}^{y} \rightarrow L_{z}^{x}$ is given by the pair of matrices

$$
\left(\left(\phi_{y \rightarrow x}, 0\right),\left(0, \phi_{y \rightarrow x}\right)\right),
$$

where $\phi_{y \rightarrow x}: R \rightarrow R^{\prime}$ is the algebra homomorphism that takes $x$ to $x$, $z$ to $z$, and $y$ to $x$.

The map $\tau_{2}: L_{y}^{x} L_{z}^{y} \rightarrow L_{z}^{x}$ is given by the pair of matrices

$$
\left(\left(\phi_{y \rightarrow z}, 0\right),\left(0, \phi_{y \rightarrow z}\right)\right),
$$

where $\phi_{y \rightarrow z}: R \rightarrow R^{\prime}$ is the algebra homomorphism that takes $x$ to $x$, and $y, z$ to $z$.

Proposition 19. The maps $\tau_{1}, \tau_{2}: L_{y}^{x} L_{z}^{y} \rightarrow L_{z}^{x}$ are homotopic.

Proof. Straightforward.

From now on, isomorphism means isomorphism in the homotopy category of factorizations, unless specified otherwise. Since $\tau_{1}, \tau_{2}$ are homotopic, they describe the same morphism in the homotopy category, denoted $\tau_{y}$. 
Let $\tau_{y}^{\prime}: L_{z}^{x} \rightarrow L_{y}^{x} L_{z}^{y}$ be given by the pair of matrices

$$
\tau_{y}^{\prime}=\left(\left(\begin{array}{c}
1 \\
-e_{x y z}
\end{array}\right),\left(\begin{array}{l}
1 \\
1
\end{array}\right)\right) \text {, where } e_{x y z}=\sum_{i+j+k=n-1} x^{i} y^{j} z^{k} .
$$

Proposition 20. $\tau_{y}$ and $\tau_{y}^{\prime}$ are mutually inverse isomorphisms in the homotopy category of factorizations with potential $w(x)-w(z)$.

Proof. It is clear that $\tau_{y}^{\prime}$ is a homomorphism of factorizations, and $\tau_{1} \tau_{y}^{\prime}$ is the identity endomorphism of $L_{z}^{x}(c)$. The proposition follows, since $\tau_{y}=\tau_{1}$ is an isomorphism of factorizations.

Proposition 21. $\tau$ is associative: there is an equality

$$
\tau_{z}\left(\tau_{y} \otimes \mathrm{Id}\right)=\tau_{y}\left(\mathrm{Id} \otimes \tau_{z}\right)
$$

of maps $L_{y}^{x} L_{z}^{y} L_{w}^{z} \rightarrow L_{w}^{x}$.

Proof. Direct computation.

Corollary 9. $\tau^{\prime}$ is associative.

COROLlary 10. For any $m$ and $k$ there is a canonical isomorphism of factorizations

$$
L_{z_{1}}^{x} L_{z_{2}}^{z_{1}} \ldots L_{z_{m}}^{z_{m-1}} L_{y}^{z_{m}} \cong L_{v_{1}}^{x} L_{v_{2}}^{v_{1}} \ldots L_{v_{k}}^{v_{k-1}} L_{y}^{v_{k}} .
$$

These isomorphisms are consistent.

$L_{y}^{x} L_{z}^{y}$ is depicted by two arcs glued together along matching endpoints, with a mark at the gluing point. The morphism $\tau_{y}$ corresponds to removing a mark, and $\tau_{y}^{\prime}$ to adding a mark (see Figure 23).

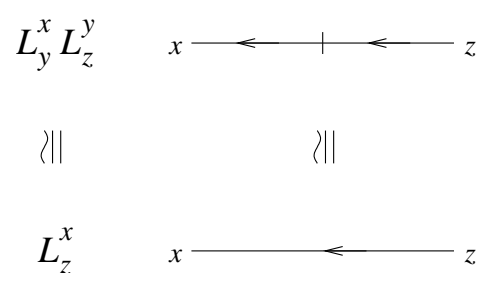

Fig. 23. Adding or removing a mark

Proposition 20 says that removing a marked point on an arc does not change the isomorphism class of a factorization, Proposition 21 says that arc removal is associative (Figure 24), while Corollary 10 asserts that two arcs, each with an arbitrary number of marks, are canonically isomorphic (Figure 25).

Denote by $L_{x}^{x}$ the quotient of $L_{y}^{x}$ by the relation $y=x$. Then $L_{x}^{x}$ is a 2-complex of $\mathbb{Q}[[x]]$-modules

$$
\mathbb{Q}[[x]] \stackrel{\pi_{x x}}{\longrightarrow} \mathbb{Q}[[x]] \stackrel{0}{\longrightarrow} \mathbb{Q}[[x]],
$$




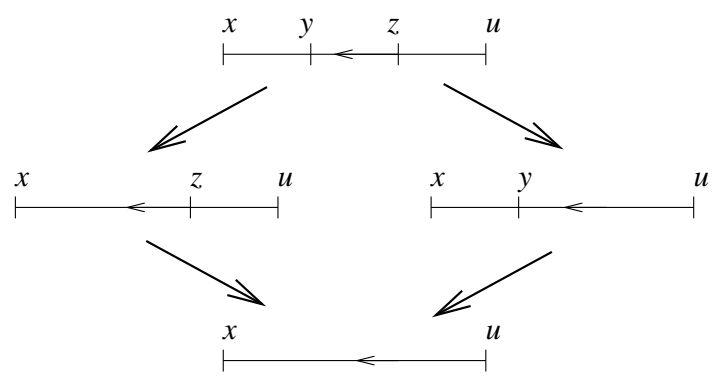

Fig. 24. Associativity of mark removal

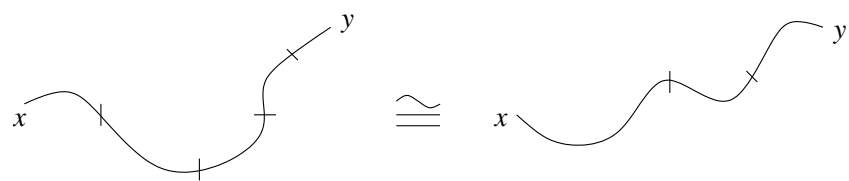

Fig. 25. Canonical isomorphism of marked arcs

and has cohomology only in degree 1 . We depict $L_{x}^{x}$ by an oriented circle with one mark $x$.

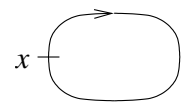

Fig. 26. 2-complex $L_{x}^{x}$

Let

$$
A:=\mathrm{H}^{*}\left(\mathbb{C P}^{n-1}, \mathbb{Q}\right)=\mathbb{Q}[X] /\left(X^{n}\right),
$$

and $\iota: \mathbb{Q} \rightarrow A$ be the unit map, $\iota(1)=1$. Choose a nonzero rational number $\zeta$, and define $\varepsilon: A \rightarrow \mathbb{Q}$ as the trace map

$$
\varepsilon\left(X^{n-1}\right)=\zeta, \quad \varepsilon\left(X^{i}\right)=0 \quad \text { if } i \neq n-1 .
$$

We identify the Milnor ring $R_{w(x)} \cong \mathbb{Q}[x] /\left(x^{n}\right)$ with $A$ by taking $x^{i} \in R_{w}$ to $X^{i} \in A$. To an oriented circle without marks we associate the 2 -periodic complex $0 \rightarrow A \rightarrow 0$, denoted $A\langle 1\rangle$.

We fix an isomorphism $\nu_{x}: A\langle 1\rangle \cong L_{x}^{x}$ of 2-periodic complexes of vector spaces, up to homotopies, by taking $X^{i} \in A$ to $x^{i} \in \mathbb{Q}[[x]] \cong\left(L_{x}^{x}\right)^{1}$ for $0 \leq i \leq n-1$. Graphically, this isomorphism means adding a mark to a circle without marks (see Figure 27).

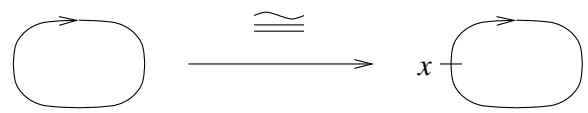

Fig. 27. Adding a mark to a circle 


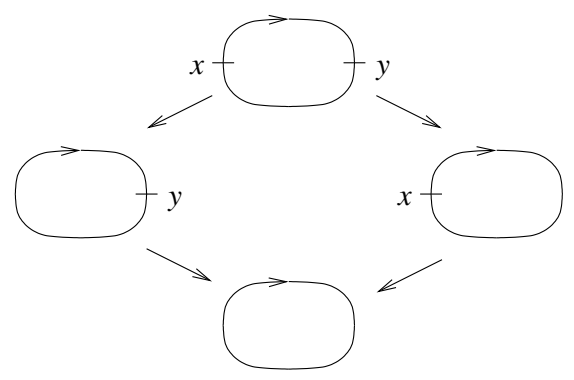

Fig. 28. Commutativity of mark removal

It is easy to see that the maps

$$
L_{y}^{x} L_{x}^{y} \stackrel{\tau_{y}}{\longrightarrow} L_{x}^{x} \stackrel{\nu_{x}^{-1}}{\longrightarrow} A\langle 1\rangle \quad \text { and } \quad L_{y}^{x} L_{x}^{y} \stackrel{\tau_{x}}{\longrightarrow} L_{y}^{y} \stackrel{\nu_{y}^{-1}}{\longrightarrow} A\langle 1\rangle
$$

are homotopic. This implies consistency between two ways to remove two marks from a circle (Figure 28).

Combining with the associativity property for mark removal and addition, we conclude that the factorizations assigned to two circles with arbitrary number of marks are canonically isomorphic in the homotopy category of 2-complexes of $\mathbb{Q}$-vector spaces (Figure 29).

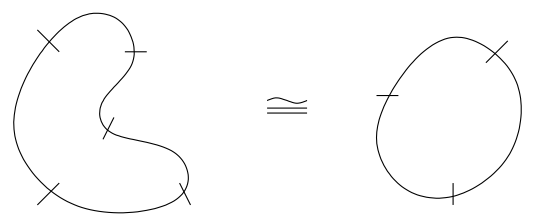

Fig. 29. Canonical isomorphism of 2-periodic complexes assigned to marked circles

REMARK. This canonical isomorphism allows us to view $\iota$ and $\varepsilon$ as maps between $\mathbb{Q}$ and the 2-complex assigned to a circle with an arbitrary number of marks.

Suppose we have a collection of factorizations $M_{i}, i \in I$, each with potential $w_{i}$ which is a signed sum of $x_{j}^{n+1}$ for $j$ in a subset of $I$. Let us tensor $M_{i}$ 's and a number of arc factorizations $L$ together in some way to produce a network of factorizations (as in Figure 30 top left). Each arc in the network has potential $x^{n+1}$. We divide arcs into internal and external arcs. External arcs are those with at least one loose endpoint. The resulting tensor product $Z_{1}=\bigotimes_{i} M_{i}$ is a factorization with potential which is a signed sum of $x_{j}^{n+1}$, over all loose endpoints $j$. The sign is determined by the orientation of the network near $j$.

Suppose now we tensor $M_{i}$ 's and several arc factorizations together so as to produce the same network but, possibly, with different marks (as in Figure 30 top right). Denote this tensor product by $Z_{2}$. Our results imply 
Proposition 22. The factorizations $Z_{1}$ and $Z_{2}$ are canonically isomorphic (in the homotopy category). The isomorphism is natural in $M_{i}$ 's, in the following sense. If $f: M_{r} \rightarrow N_{r}$ is a homomorphism of factorizations, for some $r$, and $Z_{1}^{\prime}$ (respectively, $Z_{2}^{\prime}$ ) is obtained from $Z_{1}$ (respectively, $Z_{2}$ ) by substituting $N_{r}$ for $M_{r}$ in the tensor product, then the diagram

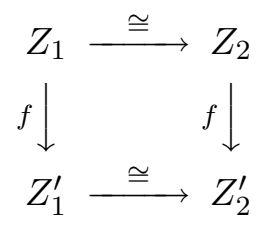

commutes (see Figure 30).

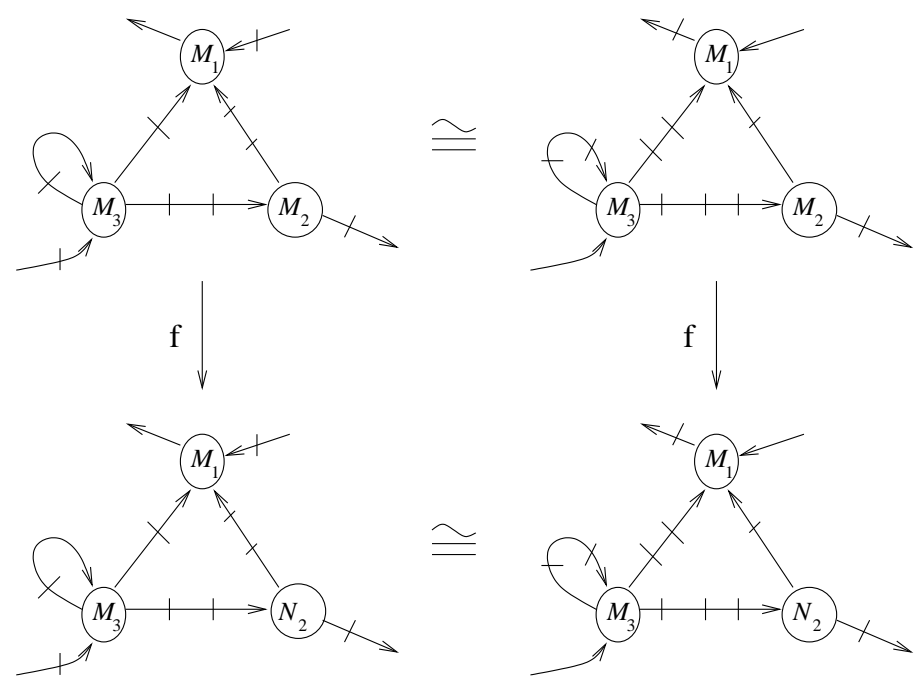

Fig. 30. Canonical isomorphism of networks; a map $f: M_{2} \rightarrow N_{2}$ induces a homomorphism of factorizations assigned to whole networks; the diagram is commutative.

Realization of the identity functor (multi-variable case). Given a multivariable potential $w=w\left(x_{1}, \ldots, x_{k}\right)$, we can write

$$
w(x)-w(y)=\sum_{i=1}^{k} w_{i}\left(x_{i}-y_{i}\right)
$$

where

$$
w_{i}=\frac{w\left(y_{1}, \ldots, y_{i-1}, x_{i}, \ldots, x_{k}\right)-w\left(y_{1}, \ldots, y_{i}, x_{i+1}, \ldots, x_{k}\right)}{x_{i}-y_{i}}
$$

are polynomials in $x$ 's and $y$ 's. Let $L$ be the tensor product (over $i$ from 1 to $k$ ) of factorizations

$$
R \stackrel{w_{i}}{\longrightarrow} R \stackrel{x_{i}-y_{i}}{\longrightarrow} R
$$

where $R=\mathbb{Q}[[x, y]]$. 
Proposition 23. For any potential $w_{1}(z)$ and any factorization $M$ with potential $w(y)-w_{1}(z)$ the tensor product $L \otimes_{y} M$ is isomorphic in the homotopy category of factorizations to the factorization $M$ with $y$ 's relabeled as $x$ 's. The isomorphism is functorial in $M$.

Proof. Apply Proposition 9 repeatedly to exclude $y_{1}, \ldots, y_{k}$.

Proposition 23 says, essentially, that tensoring with $L$ is the identity functor. Also, we have

$$
\operatorname{Hom}_{\mathrm{HMF}}(L, L) \cong \mathbb{Q}[[x]]_{w(x)}, \quad \operatorname{Ext}_{\mathrm{HMF}}^{1}(L, L) \cong 0,
$$

i.e., the endomorphism ring of $L$ is the Jacobian algebra of $w$. Note that Theorem 1 applied to $L$ implies Proposition 4.

The results of the previous subsection and of Section 9 can be generalized from $x^{n+1}$ to arbitrary multi-variable potentials, but we postpone this analysis to a later paper.

\section{Homogeneous potentials and graded factorizations}

Homogeneous potentials and graded factorizations. Suppose that each variable $x_{i}$ is given a positive integer degree $p_{i}$ and the potential $w$ is homogeneous of degree $p$. Then $w$ belongs to the Jacobian ideal,

$$
w=\frac{1}{p} \sum_{i} p_{i} x_{i} \frac{\partial f}{\partial x_{i}}
$$

(Euler's formula), so that the Milnor and Tyurina algebras are isomorphic.

The ring of power series with coefficients in a field is local. After switching from rings to graded rings, the role of the power series rings is played by polynomial rings whose generators are in positive degrees only, since these rings have only one maximal homogeneous ideal. From now on in this paper we work with homogeneous potentials and switch from the power series ring to the ring of polynomials. From here on $R$ is a polynomial ring.

A graded (or homogeneous) factorization with a homogeneous potential $w$ of degree $2(n+1)$ consists of free graded $R$-modules $M^{0}, M^{1}$ and degree $n+1$ homomorphisms $d^{0}, d^{1}$ such that $d^{1} d^{0}=w$ (this implies $\left.d^{0} d^{1}=w\right)$,

$$
M^{0} \stackrel{d^{0}}{\longrightarrow} M^{1} \stackrel{d^{1}}{\longrightarrow} M^{0} .
$$

A homomorphism of graded factorizations is required to have degree 0 , while a homotopy should have degree $-n-1$. Each of the six categories of factorizations described in Section 3 has a graded version, denoted in lowercase letters. For instance, $\mathrm{hmf}_{w}$ is the homotopy category of graded factorizations of $w$ with finite-dimensional cohomology. This category is triangulated. 
We denote by $\{m\}$ the grading shift up by $m$. The factorization $M\{m\}$ has the form

$$
M^{0}\{m\} \stackrel{d^{0}}{\longrightarrow} M^{1}\{m\} \stackrel{d^{1}}{\longrightarrow} M^{0}\{m\} .
$$

The cohomological shift functor $\langle 1\rangle$ does not change the grading of $M^{0}, M^{1}$, and commutes with the grading shift functor $\{m\}$.

All results and constructions of Sections 2, 3 and 4 easily extend to graded factorizations. There is no need to complete tensor products of factorizations in the graded case. The factorization $\{a, b\}$ is defined for homogeneous $a, b$ with $\operatorname{deg}(a)+\operatorname{deg}(b)=2(n+1)$, and has the form

$$
R \stackrel{a}{\longrightarrow} R\{n+1-\operatorname{deg}(a)\} \stackrel{b}{\longrightarrow} R .
$$

We shifted the degree of the middle $R$ so that the differentials would have degree $n+1$. Then

$$
\{a, b\}\langle 1\rangle=\{b, a\}\{\operatorname{deg}(b)-n-1\} .
$$

Notice two different uses of curly brackets: to denote Koszul factorizations and shifts.

When extending the subsection 'Realization of the identity functor (onevariable case)' of Section 4 to the graded case, we give the variables $x, y, z$ degree 2. The factorization $L_{y}^{x}$ has the form

$$
R \stackrel{\pi_{x y}}{\longrightarrow} R\{1-n\} \stackrel{x-y}{\longrightarrow} R .
$$

The isomorphism in Proposition 15 has degree 0, assuming $M$ is a graded factorization.

The cohomology $H(M)$ of a homogeneous factorization is a $\mathbb{Z}_{2} \oplus \mathbb{Z}$-graded $\mathbb{Q}$-vector space,

$$
H(M)=\bigoplus_{i \in\{0,1\}, j \in \mathbb{Z}} H^{i, j}(M) .
$$

We define the graded dimension of $M$ as

$$
\operatorname{gdim}(M):=\sum_{j \in \mathbb{Z}, i \in\{0,1\}} \operatorname{dim} H^{i, j}(M) q^{j} s^{i} .
$$

Then, for external tensor product $M \otimes N$,

$$
\operatorname{gdim}(M \otimes N)=\operatorname{gdim}(M) \operatorname{gdim}(N),
$$

assuming the relation $s^{2}=1$. The dimension of $M$ is the $q=s=1$ specialization of the graded dimension.

The algebra $A$ (cohomology of $\mathbb{C P}^{n-1}$ ), defined in the previous section, is naturally graded. We shift the grading of $A$ down by $n-1$ so that $\operatorname{deg}(1)=$ $1-n$, and, more generally, $\operatorname{deg}\left(X^{i}\right)=2 i+1-n$. The unit map $\iota: \mathbb{Q} \rightarrow A$ and the trace map $\varepsilon: A \rightarrow \mathbb{Q}$ have degree $1-n$, while the multiplication 
in $A$ has degree $n-1$. To a circle without marks we assign the 2 -complex $A\langle 1\rangle$ of graded vector spaces.

Let $M$ be a homogeneous factorization of finite rank. If the endomorphism ring $\operatorname{Hom}_{\mathrm{mf}}(M, M)$ contains a degree 0 idempotent $e$, we can decompose $M$ into a direct sum of factorizations, $M=e M \oplus(1-e) M$. Suppose instead that the quotient ring $\operatorname{Hom}_{\mathrm{hmf}}(M, M)$ contains an idempotent $e$. Let $I$ be the kernel of the quotient map

$$
\operatorname{Hom}_{\mathrm{mf}}(M, M) \stackrel{f}{\longrightarrow} \operatorname{Hom}_{\mathrm{hmf}}(M, M),
$$

and $J$ the ideal in $\operatorname{Hom}_{\mathrm{mf}}(M, M)$ of endomorphisms that induce the zero map on cohomology. Note that $I \subset J$, and $J$ is a nilpotent ideal in $\operatorname{Hom}_{\mathrm{mf}}(M, M)$, since a degree 0 endomorphism cannot have coefficients of arbitrary large degrees. Thus, $J^{N}=0$ for some $N$, and therefore, $I^{N}=0$. Nilpotent ideals have the lifting idempotents property (exercise, or see [Be, Theorem 1.7.3]). There exists an idempotent $\widetilde{e} \in \operatorname{Hom}_{\mathrm{mf}}(M, M)$ that lifts $e$, that is, $f(\widetilde{e})=e$. It allows us to decompose $M=\widetilde{e} M \oplus(1-\widetilde{e}) M$. We state this as

Proposition 24. The category $\mathrm{hmf}_{w}$ has the splitting idempotents property.

REMARK. The category $\mathrm{HMF}_{w}$ has this property as well, which can be seen by a slight modification of the above argument, using the fact that $\bigcap_{N} J^{N}=0$ and lifting $e$ recursively.

An additive category is called Krull-Schmidt if any object has the unique decomposition property. In other words, if $M \cong \bigoplus_{i \in I} M_{i}$ and $M \cong \bigoplus_{j \in J} N_{j}$, for some sets $I, J$ and indecomposables $M_{i}, N_{j}$, then there is a bijection $z: I \rightarrow J$ such that $M_{i} \cong N_{z(i)}$.

Proposition 25. The category $\mathrm{hmf}_{w}$ is Krull-Schmidt.

Proof. Any object of $\mathrm{hmf}_{w}$ is isomorphic to a finite rank factorization. This and Proposition 24 imply that the endomorphism ring of any indecomposable in $\mathrm{hmf}_{w}$ is local. Proposition 25 follows.

For graded factorizations $M, N$ with finite-dimensional cohomology there is an isomorphism of graded $R$-modules

$$
\operatorname{Hom}_{\mathrm{HMF}}(M, N)=\bigoplus_{i \in \mathbb{Z}} \operatorname{Hom}_{\mathrm{hmf}}(M\{i\}, N) .
$$

Given a graded cyclic Koszul factorization $\{\mathbf{a}, \mathbf{b}\}$, its graded dual is

$$
\{\mathbf{a}, \mathbf{b}\} \bullet\{-\mathbf{b}, \mathbf{a}\} \cong\{\mathbf{a},-\mathbf{b}\}\langle m\rangle\left\{\sum_{i} \operatorname{deg}\left(a_{i}\right)-m(n+1)\right\},
$$

where the sequences $\mathbf{a}$ and $\mathbf{b}$ have length $m$. 
Quasi-homogeneous potentials. A potential that is homogeneous with respect to some basis in $R$ is called quasi-homogeneous. A quasi-homogeneous potential lies in its Jacobian ideal. From the work of K. Saito [Sa] we know that the converse is true: only quasi-homogeneous potentials lie in their Jacobian ideal (so that the Milnor and Tyurina algebras are isomorphic). To a mathematician, quasi-homogeneous singularities are distinguished by the existence of the associated Frobenius manifold [BV], [D], and other special properties $[\mathrm{AGV}],[\mathrm{Di}],[\mathrm{Ku}]$. From a string theorist's viewpoint, each quasi-homogeneous singularity gives rise to the rich structure of a (super) conformal 2D field theory [VW], [M], while an arbitrary isolated singularity only produces a 2D topological field theory, the latter equivalent to a commutative Frobenius algebra (a 0-dimensional Gorenstein ring).

In the rest of the paper we are dealing exclusively with homogeneous potentials.

\section{Planar graphs and factorizations}

Factorization from a planar graph. We consider graphs of a particular kind embedded in a disc (for an example see Figure 7). A graph can have both unoriented and oriented edges, and oriented loops. Unoriented edges are called "wide" and depicted correspondingly. Any unoriented edge has two oriented edges entering it at one vertex, and two oriented edges leaving it at the other. Oriented edges might end on the boundary of the disc. Inside the disc we allow only trivalent vertices where a wide edge and a pair of oriented edges meet. An oriented edge is internal if none of its endpoints is on the boundary of the disc. Otherwise, the edge is called external. Any internal edge has one or more marks placed on it. We also treat boundary points as marks; this ensures that each external edge has a mark. Additional marks on external edges are allowed. An oriented loop may or may not have marks.

If $\Gamma$ is such a graph, we denote by $m(\Gamma)$ the set of its marks and by $\partial \Gamma$ the set of boundary points, the latter a subset of $m(\Gamma)$. If $i \in \partial \Gamma$, the sign of $i$, denoted $s(i)$, is 1 if the edge at $i$ is oriented outward, and -1 if the edge is oriented inward. For instance, boundary points marked 1,2, 7 in Figure 7 have sign 1 , and points $4,8,9$ have sign -1 .

Let $R=\mathbb{Q}\left[x_{i}: i \in m(\Gamma)\right]$ be the ring of polynomials in variables $x_{i}$, over all marks $i$, and $R^{\prime}$ be its subring $\mathbb{Q}\left[x_{i}: i \in \partial \Gamma\right]$. We introduce a grading on $R$ and $R^{\prime}$ by giving each $x_{i}$ degree 2 .

Assign to $\Gamma$ the potential

$$
w(\Gamma)=\sum_{i \in \partial \Gamma} s(i) x_{i}^{n+1} .
$$


To $\Gamma$ we now associate a graded factorization $C(\Gamma)$ over the ring $R^{\prime}$ with potential $w(\Gamma)$. First, to a wide edge $t$ bounded by marks 1,2,3, 4 as in Figure 5 we assign a factorization with potential

$$
w_{t}=x_{1}^{n+1}+x_{2}^{n+1}-x_{3}^{n+1}-x_{4}^{n+1} .
$$

Starting with formal variables $x, y$, we can write $x^{n+1}+y^{n+1}$ as a polynomial in $x+y$ and $x y$. Let $g$ be this polynomial,

$$
g(x+y, x y)=x^{n+1}+y^{n+1} .
$$

Explicitly,

$$
g\left(s_{1}, s_{2}\right)=s_{1}^{n+1}+(n+1) \sum_{1 \leq i \leq(n+1) / 2} \frac{(-1)^{i}}{i}\left(\begin{array}{c}
n-i \\
i-1
\end{array}\right) s_{2}^{i} s_{1}^{n+1-2 i} .
$$

$w_{t}$ can be written as

$$
\begin{aligned}
w_{t}= & g\left(x_{1}+x_{2}, x_{1} x_{2}\right)-g\left(x_{3}+x_{4}, x_{3} x_{4}\right) \\
= & g\left(x_{1}+x_{2}, x_{1} x_{2}\right)-g\left(x_{3}+x_{4}, x_{1} x_{2}\right) \\
& +g\left(x_{3}+x_{4}, x_{1} x_{2}\right)-g\left(x_{3}+x_{4}, x_{3} x_{4}\right) \\
= & \frac{g\left(x_{1}+x_{2}, x_{1} x_{2}\right)-g\left(x_{3}+x_{4}, x_{1} x_{2}\right)}{x_{1}+x_{2}-x_{3}-x_{4}}\left(x_{1}+x_{2}-x_{3}-x_{4}\right) \\
& \quad+\frac{g\left(x_{3}+x_{4}, x_{1} x_{2}\right)-g\left(x_{3}+x_{4}, x_{3} x_{4}\right)}{x_{1} x_{2}-x_{3} x_{4}}\left(x_{1} x_{2}-x_{3} x_{4}\right) .
\end{aligned}
$$

Let

$$
\begin{aligned}
& u_{1}=u_{1}\left(x_{1}, x_{2}, x_{3}, x_{4}\right)=\frac{g\left(x_{1}+x_{2}, x_{1} x_{2}\right)-g\left(x_{3}+x_{4}, x_{1} x_{2}\right)}{x_{1}+x_{2}-x_{3}-x_{4}}, \\
& u_{2}=u_{2}\left(x_{1}, x_{2}, x_{3}, x_{4}\right)=\frac{g\left(x_{3}+x_{4}, x_{1} x_{2}\right)-g\left(x_{3}+x_{4}, x_{3} x_{4}\right)}{x_{1} x_{2}-x_{3} x_{4}} .
\end{aligned}
$$

Note that $u_{1}$ and $u_{2}$ are polynomials, and

$$
w_{t}=u_{1}\left(x_{1}+x_{2}-x_{3}-x_{4}\right)+u_{2}\left(x_{1} x_{2}-x_{3} x_{4}\right) .
$$

To $t$ we assign the graded factorization

$$
C_{t}:=\left\{\left(u_{1}, u_{2}\right),\left(x_{1}+x_{2}-x_{3}-x_{4}, x_{1} x_{2}-x_{3} x_{4}\right)\right\}\{-1\} .
$$

In other words, $C_{t}$ is the tensor product of graded factorizations

$$
R_{t} \stackrel{u_{1}}{\longrightarrow} R_{t}\{1-n\} \stackrel{x_{1}+x_{2}-x_{3}-x_{4}}{\longrightarrow} R_{t}
$$

and

$$
R_{t} \stackrel{u_{2}}{\longrightarrow} R_{t}\{3-n\} \stackrel{x_{1} x_{2}-x_{3} x_{4}}{\longrightarrow} R_{t},
$$

with the grading shifted down by 1 .

In general, a wide edge $t$ will be bounded by marks $i, j, k, l$. Then $C_{t}$ is defined as above, with $1,2,3,4$ converted into $i, j, k, l$. 
If $\alpha$ is an arc in an oriented edge (or in an oriented loop) bounded by marks $i$ and $j$ and oriented from $j$ to $i$, we denote by $L_{j}^{i}$ the factorization

$$
R_{\alpha} \stackrel{\pi_{i j}}{\longrightarrow} R_{\alpha} \stackrel{x_{i}-x_{j}}{\longrightarrow} R_{\alpha}
$$

where $R_{\alpha}=\mathbb{Q}\left[x_{i}, x_{j}\right]$ and

$$
\pi_{i j}=\frac{x_{i}^{n+1}-x_{j}^{n+1}}{x_{i}-x_{j}} .
$$

This factorization was introduced earlier, in Section 4, as the factorization assigned to an arc.

To an oriented loop without marks we assign the 2-complex $A\langle 1\rangle$ (see Section 4).

Finally, we define $C(\Gamma)$ as the tensor product of $C_{t}$ over all wide edges $t$, of $L_{j}^{i}$ over all $\alpha$, and of $A\langle 1\rangle$ over all markless loops in $\Gamma$. The tensor product is formed over suitable intermediate rings so that $C(\Gamma)$ is a free module of finite rank over $R$. For instance, to form $C(\Gamma)$ for $\Gamma$ in Figure 7, we first tensor $C_{t_{1}}$ with $L_{5}^{3}$ over the ring $\mathbb{Q}\left[x_{3}\right]$, and then tensor the result with $C_{t_{2}}$ over $\mathbb{Q}\left[x_{5}\right]$. In conclusion, we tensor $C_{t_{1}} \otimes L_{5}^{3} \otimes C_{t_{2}}$ with $L_{6}^{7}$ over $\mathbb{Q}\left[x_{6}\right]$.

Properties. Clearly, $C(\Gamma)$ is a factorization with potential $w(\Gamma)$. We treat it as a graded factorization over the ring $R^{\prime}$ of polynomials in boundary variables. $w(\Gamma)$ is a nondegenerate potential in this ring. If a graph has at least one internal mark, $C(\Gamma)$ has infinite rank as an $R^{\prime}$-module.

Proposition 26. For any graph $\Gamma$, the factorization $C(\Gamma)$ lies in $\operatorname{hmf}_{w(\Gamma)}$.

Proof. In other words, $C(\Gamma)$ has finite-dimensional cohomology. This follows at once from results of Section 3.

Suppose that $\Gamma^{\prime}$ is obtained from $\Gamma$ by placing a different collection of internal marks on oriented edges and loops of $\Gamma$. Then the two graphs have the same potential $w\left(\Gamma^{\prime}\right)=w(\Gamma)$ assigned to them, and factorization $C\left(\Gamma^{\prime}\right)$ belongs to the category $\mathrm{hmf}_{w(\Gamma)}$ since the graphs share the same set of boundary points.

Proposition 27. There is a canonical isomorphism in $\mathrm{hmf}_{w(\Gamma)}$

$$
C\left(\Gamma^{\prime}\right) \cong C(\Gamma) \text {. }
$$

Proof. This is a special case of Proposition 22.

Maps $\chi_{0}$ and $\chi_{1}$. Consider the graphs $\Gamma^{0}, \Gamma^{1}$ depicted in Figure 8. The factorization $C\left(\Gamma^{0}\right)$ is the tensor product of the factorizations $L_{4}^{1}$ and $L_{3}^{2}$, and is given by

$$
\left(\begin{array}{c}
R(\emptyset) \\
R(a b)\{2-2 n\}
\end{array}\right) \stackrel{P_{0}}{\longrightarrow}\left(\begin{array}{c}
R(a)\{1-n\} \\
R(b)\{1-n\}
\end{array}\right) \stackrel{P_{1}}{\longrightarrow}\left(\begin{array}{c}
R(\emptyset) \\
R(a b)\{2-2 n\}
\end{array}\right)
$$


where

$$
\begin{gathered}
P_{0}=\left(\begin{array}{cc}
\pi_{14} & x_{2}-x_{3} \\
\pi_{23} & x_{4}-x_{1}
\end{array}\right), \quad P_{1}=\left(\begin{array}{cc}
x_{1}-x_{4} & x_{2}-x_{3} \\
\pi_{23} & -\pi_{14}
\end{array}\right), \\
\pi_{i j}=\sum_{k=0}^{n} x_{i}^{k} x_{j}^{n-k},
\end{gathered}
$$

and we assigned labels $a, b$ to $L_{4}^{1}$ and $L_{3}^{2}$. The factorization $C\left(\Gamma^{1}\right)$ is

$$
\left(\begin{array}{c}
R(\emptyset)\{-1\} \\
R\left(a^{\prime} b^{\prime}\right)\{3-2 n\}
\end{array}\right) \stackrel{Q_{1}}{\longrightarrow}\left(\begin{array}{c}
R\left(a^{\prime}\right)\{-n\} \\
R\left(b^{\prime}\right)\{2-n\}
\end{array}\right) \stackrel{Q_{2}}{\longrightarrow}\left(\begin{array}{c}
R(\emptyset)\{-1\} \\
R\left(a^{\prime} b^{\prime}\right)\{3-2 n\}
\end{array}\right),
$$

with

$$
\begin{aligned}
Q_{1} & =\left(\begin{array}{cc}
u_{1} & x_{1} x_{2}-x_{3} x_{4} \\
u_{2} & x_{3}+x_{4}-x_{1}-x_{2}
\end{array}\right), \\
Q_{2} & =\left(\begin{array}{cc}
x_{1}+x_{2}-x_{3}-x_{4} & x_{1} x_{2}-x_{3} x_{4} \\
u_{2} & -u_{1}
\end{array}\right) .
\end{aligned}
$$

We assigned labels $a^{\prime}, b^{\prime}$ to the two factorizations (with degenerate potentials) whose tensor product is $C\left(\Gamma^{1}\right)$.

A map between $C\left(\Gamma^{0}\right)$ and $C\left(\Gamma^{1}\right)$ can be described by a pair of $2 \times 2$ matrices $\left(U_{0}, U_{1}\right)$. Let $\chi_{0}: C\left(\Gamma^{0}\right) \rightarrow C\left(\Gamma^{1}\right)$ be given by the pair

$$
\begin{aligned}
& U_{0}=\left(\begin{array}{cc}
x_{4}-x_{2}+\mu\left(x_{1}+x_{2}-x_{3}-x_{4}\right) & 0 \\
a_{1} & 1
\end{array}\right), \\
& U_{1}=\left(\begin{array}{cc}
x_{4}+\mu\left(x_{1}-x_{4}\right) & \mu\left(x_{2}-x_{3}\right)-x_{2} \\
-1 & 1
\end{array}\right),
\end{aligned}
$$

where

$$
a_{1}=(\mu-1) u_{2}+\frac{u_{1}+x_{1} u_{2}-\pi_{23}}{x_{1}-x_{4}}
$$

and $\mu \in \mathbb{Z}$. Different choices of $\mu$ give homotopic maps. The map $\chi_{0}$ has degree 1.

Let $\chi_{1}: C\left(\Gamma^{1}\right) \rightarrow C\left(\Gamma^{0}\right)$ be given by the pair of matrices $\left(V_{0}, V_{1}\right)$ :

$$
V_{0}=\left(\begin{array}{cc}
1 & 0 \\
a_{2} & a_{3}
\end{array}\right), \quad V_{1}=\left(\begin{array}{cc}
1 & x_{3}+\lambda\left(x_{2}-x_{3}\right) \\
1 & x_{1}+\lambda\left(x_{4}-x_{1}\right)
\end{array}\right),
$$

where

$$
a_{2}=\lambda u_{2}+\frac{u_{1}+x_{1} u_{2}-\pi_{23}}{x_{4}-x_{1}}, \quad a_{3}=\lambda\left(x_{3}+x_{4}-x_{1}-x_{2}\right)+x_{1}-x_{3}
$$

and $\lambda \in \mathbb{Z}$. Different choices of $\lambda$ give homotopic maps. The map $\chi_{1}$ has degree 1 . 
The composition $\chi_{1} \chi_{0}$ is described by the pair $\left(V_{0} U_{0}, V_{1} U_{1}\right)$. Computing the products and specializing to $\mu=1-\lambda$ we get

$$
V_{0} U_{0}=V_{1} U_{1}=\left(x_{1}-x_{3}+\lambda\left(x_{3}+x_{4}-x_{1}-x_{2}\right)\right) \mathrm{I},
$$

where $\mathrm{I}$ is the identity $2 \times 2$ matrix. Therefore, the composition $\chi_{1} \chi_{0}$ is homotopic to the multiplication by the $x_{1}-x_{3}$ endomorphism of $C\left(\Gamma^{0}\right)$,

$$
\chi_{1} \chi_{0}=m\left(x_{1}-x_{3}\right) .
$$

This is obtained by setting $\lambda=0$ in (20). Choosing instead $\lambda=1$, we see that $\chi_{1} \chi_{0}$ is homotopic to multiplication by $x_{4}-x_{2}$. There is no contradiction here, since the endomorphism of multiplication by $x_{1}+x_{2}-x_{3}-x_{4}$ is null-homotopic.

Likewise, the composition $\chi_{0} \chi_{1}$ is homotopic to the multiplication by $x_{1}-x_{3}$ (and to the multiplication by $x_{4}-x_{2}$ ) endomorphism of $C\left(\Gamma^{1}\right)$.

Disjoint union. Given two graphs $\Gamma_{1}, \Gamma_{2}$ with potentials $w_{1}, w_{2}$, the potential of their disjoint union $\Gamma_{1} \sqcup \Gamma_{2}$ is the exterior sum $w_{1}+w_{2}$. An example of a disjoint union is depicted in Figure 31. More often than not, this operation is not uniquely determined by $\Gamma_{1}$ and $\Gamma_{2}$, since we can place $\Gamma_{2}$ between any pair of adjacent exterior legs of $\Gamma_{1}$. When $\Gamma_{2}$ has no exterior legs, we can place it inside any region of $\Gamma_{1}$, including those not adjacent to the border of the disc.

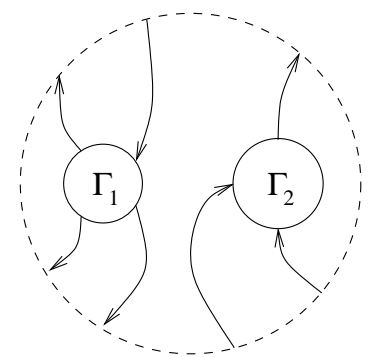

Fig. 31. A disjoint union of $\Gamma_{1}$ and $\Gamma_{2}$

Proposition 28. There is a canonical isomorphism in $\mathrm{hmf}_{w_{1}+w_{2}}$

$$
C\left(\Gamma_{1} \sqcup \Gamma_{2}\right) \cong C\left(\Gamma_{1}\right) \otimes_{\mathbb{Q}} C\left(\Gamma_{2}\right) .
$$

Proof. This is obvious from the definition of $C(\Gamma)$.

Corollary 11. If $\Gamma_{2}$ is a disjoint union of $\Gamma_{1}$ and a loop, then

$$
C\left(\Gamma_{2}\right) \cong C\left(\Gamma_{1}\right)\langle 1\rangle \otimes_{\mathbb{Q}} A .
$$

Direct sum decomposition I. For the graphs $\Gamma_{1}, \Gamma$ depicted in Figure 32, $C\left(\Gamma_{1}\right), C(\Gamma)$ are $\left(R^{\prime}, w\right)$-factorizations, where $R^{\prime}=\mathbb{Q}\left[x_{2}, x_{3}\right]$ and $w=$ $x_{2}^{n+1}-x_{3}^{n+1}$. Notice that $C(\Gamma)$ has infinite rank as a $\left(R^{\prime}, w\right)$-factorization. 

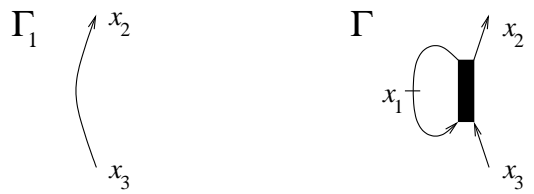

Fig. 32. Graphs $\Gamma_{1}$ and $\Gamma$

It can also be viewed as a factorization over the larger ring $\mathbb{Q}\left[x_{1}, x_{2}, x_{3}\right]$ but with a degenerate potential.

Proposition 29. There is an isomorphism in $\mathrm{hmf}_{w}$

$$
C(\Gamma)\langle 1\rangle \cong \bigoplus_{i=0}^{n-2} C\left(\Gamma_{1}\right)\{2-n+2 i\} .
$$

Proof. Let $\Gamma_{2}$ be the disjoint union of $\Gamma_{1}$ and a circle with one mark (see Figure 33). Define grading-preserving maps

$$
\alpha: C\left(\Gamma_{1}\right)\langle 1\rangle \rightarrow C(\Gamma)\{n-2\}, \quad \beta: C(\Gamma)\{2-n\} \rightarrow C\left(\Gamma_{1}\right)\langle 1\rangle
$$

as follows. $\alpha$ is the composition (Figure 33)

$$
C\left(\Gamma_{1}\right)\langle 1\rangle \stackrel{\iota^{\prime}}{\longrightarrow} C\left(\Gamma_{2}\right)\{n-1\} \stackrel{\chi_{0}}{\longrightarrow} C(\Gamma)\{n-2\},
$$

where $\iota^{\prime}$ is the tensor product of the identity of $C\left(\Gamma_{1}\right)$ with the "unit" map $\iota$.

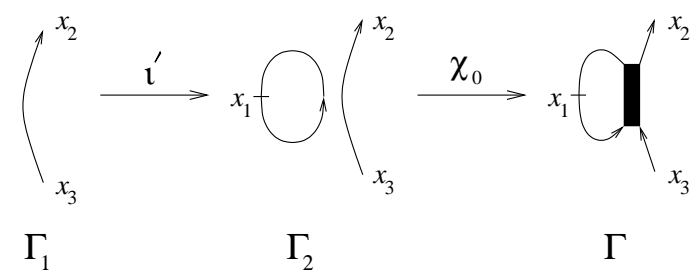

Fig. 33. Map $\alpha$

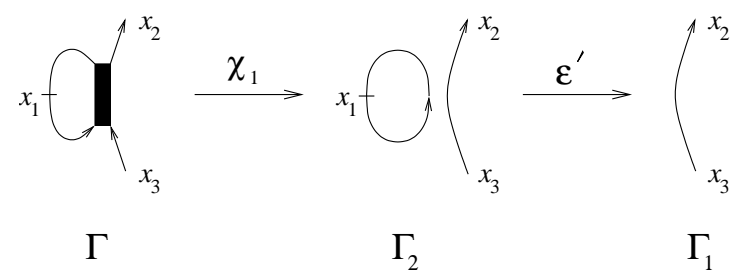

Fig. 34. Map $\beta$

$\beta$ is the composition (Figure 34)

$$
C(\Gamma)\{2-n\} \stackrel{\chi_{1}}{\longrightarrow} C\left(\Gamma_{2}\right)\{1-n\} \stackrel{\varepsilon^{\prime}}{\longrightarrow} C\left(\Gamma_{1}\right)\langle 1\rangle,
$$

where $\varepsilon^{\prime}$ is the product of the identity of $C\left(\Gamma_{1}\right)$ with the trace map $\varepsilon$. 
Define grading-preserving maps $\alpha_{i}, \beta_{i}$ for $0 \leq i \leq n-2$ by

$$
\begin{aligned}
& \alpha_{i}: C\left(\Gamma_{1}\right)\langle 1\rangle \rightarrow C(\Gamma)\{n-2-2 i\}, \quad \alpha_{i}=\sum_{j=0}^{i} m\left(x_{1}^{j} x_{2}^{i-j}\right) \alpha, \\
& \beta_{i}: C(\Gamma)\{n-2-2 i\} \rightarrow C\left(\Gamma_{1}\right)\langle 1\rangle, \quad \beta_{i}=\beta m\left(x_{1}^{n-i-2}\right) .
\end{aligned}
$$

Here $m\left(x_{1}^{j} x_{2}^{i-j}\right)$ denotes the endomorphism of $C(\Gamma)$ which is the multiplication by $x_{1}^{j} x_{2}^{i-j}$.

Lemma 1. $\beta_{j} \alpha_{i}=\delta_{i, j} \zeta$ Id.

Proof. We have

$$
\begin{aligned}
\beta_{j} \alpha_{i}= & \sum_{k=0}^{i} \beta m\left(x_{1}^{n-j-2}\right) m\left(x_{1}^{k} x_{2}^{i-k}\right) \alpha=\sum_{k=0}^{i} \varepsilon^{\prime} \chi_{1} \chi_{0} m\left(x_{1}^{n-j-2}\right) m\left(x_{1}^{k} x_{2}^{i-k}\right) \iota^{\prime} \\
= & \sum_{k=0}^{i} \varepsilon^{\prime} m\left(x_{1}-x_{3}\right) m\left(x_{1}^{n+k-j-2} x_{2}^{i-k}\right) \iota^{\prime} \\
= & \sum_{k=0}^{i} \varepsilon^{\prime} m\left(x_{1}-x_{2}\right) m\left(x_{1}^{n+k-j-2} x_{2}^{i-k}\right) \iota^{\prime} \\
= & \sum_{k=0}^{i} \varepsilon^{\prime} m\left(x_{1}-x_{2}\right) m\left(x_{1}^{n+k-j-2} x_{2}^{i-k}\right) \iota^{\prime} \\
= & \varepsilon^{\prime} m\left(x_{1}^{n+i-j-1}-x_{1}^{n-j-2} x_{2}^{i+1}\right) \iota^{\prime}=\varepsilon^{\prime} m\left(x_{1}^{n+i-j-1}\right) \iota^{\prime} \\
= & \varepsilon\left(X^{n+i-j-1}\right) \operatorname{Id}=\delta_{i, j} \zeta \mathrm{Id} .
\end{aligned}
$$

In the third equality we used the fact that $\chi_{1} \chi_{0}=m\left(x_{1}-x_{3}\right)$, and in the fourth that $m\left(x_{2}\right)=m\left(x_{3}\right)$ as endomorphisms of $C\left(\Gamma_{2}\right)$. Recall that $\zeta=\varepsilon\left(X^{n-1}\right)$ is a nonzero rational number.

The lemma implies that the map

$$
\alpha^{\prime}=\sum_{i=0}^{n-2} \alpha_{i}: \bigoplus_{i=0}^{n-2} C\left(\Gamma_{1}\right)\langle 1\rangle\{2+2 i-n\} \rightarrow C(\Gamma)
$$

is an inclusion of a direct summand, since $\beta^{\prime} \alpha^{\prime}=\mathrm{Id}$, where

$$
\beta^{\prime}=\sum_{i=0}^{n-2} \beta_{i}: C(\Gamma) \rightarrow \bigoplus_{i=0}^{n-2} C\left(\Gamma_{1}\right)\langle 1\rangle\{2+2 i-n\} .
$$

In particular, $\alpha^{\prime}$ induces an injective map $H\left(\alpha^{\prime}\right)$ on cohomology of these factorizations. The factorization $C\left(\Gamma_{1}\right)$ has dimension 2 , and the factorization on the left hand side of $(21)$ has dimension $2(n-1)$. To finish the proof of Proposition 29 it suffices to show that $H\left(\alpha^{\prime}\right)$ is bijective, which, in turn, follows from the following lemma. 
Lemma 2. The factorization $C(\Gamma)$ has dimension $2(n-1)$.

Proof. The quotient of $C(\Gamma)$ by the ideal $\left(x_{2}, x_{3}\right)$ is a 2 -complex, and a free module of rank 4 over the ring $R_{1}=\mathbb{Q}\left[x_{1}\right]$. It is the cyclic Koszul complex of the pair $\left\{\left((n+1) x_{1}^{n},-(n+1) x_{1}^{n-1}\right),(0,0)\right\}$, since

$$
\left.u_{1}\right|_{x_{1}=x_{4}, x_{2}=0, x_{3}=0}=(n+1) x_{1}^{n},\left.\quad u_{2}\right|_{x_{1}=x_{4}, x_{2}=0, x_{3}=0}=-(n+1) x_{1}^{n-1} .
$$

By changing coordinates using a suitable automorphism $\gamma$ of $N=R_{1} \oplus R_{1}$, as explained at the end of Section 2, we see that this 2-complex is isomorphic to the cyclic Koszul complex of the pair $\left\{\left(x_{1}^{n_{1}}, 0\right),(0,0)\right\}$. The complex

$$
\left\{x_{1}^{n-1}, 0\right\}: R_{1} \stackrel{x_{1}^{n-1}}{\longrightarrow} R_{1} \stackrel{0}{\longrightarrow} R_{1}
$$

has cohomology of dimension $n-1$. Tensoring it with $\{0,0\}$ doubles the dimension. Thus,

$$
\operatorname{dim} H(C(\Gamma))=\operatorname{dim} H\left(\left\{\left(x_{1}^{n-1}, 0\right),(0,0)\right\}\right)=2(n-1) .
$$

Proposition 29 follows.
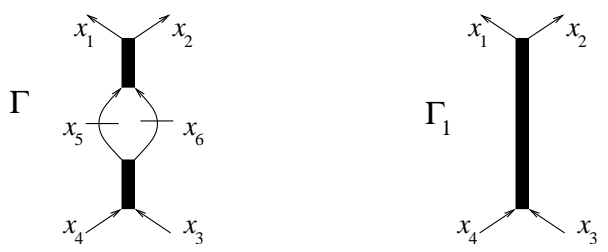

Fig. 35

Direct sum decomposition II. Consider factorizations $C(\Gamma), C\left(\Gamma_{1}\right)$, where $\Gamma, \Gamma_{1}$ are now the graphs depicted in Figure 35. Both $C(\Gamma), C\left(\Gamma_{1}\right)$ are $\left(R^{\prime}, w\right)$-factorizations, where $R^{\prime}=\mathbb{Q}\left[x_{1}, x_{2}, x_{3}, x_{4}\right]$ and

$$
w=x_{1}^{n+1}+x_{2}^{n+1}-x_{3}^{n+1}-x_{4}^{n+1} .
$$

The factorization $C(\Gamma)$ has infinite rank.

Proposition 30. There is an isomorphism in $\mathrm{hmf}_{w}$

$$
C(\Gamma) \cong C\left(\Gamma_{1}\right)\{1\} \oplus C\left(\Gamma_{1}\right)\{-1\} .
$$

Proof. Let $R=\mathbb{Q}\left[x_{1}, \ldots, x_{6}\right], s_{1}=x_{5}+x_{6}, s_{2}=x_{5} x_{6}$ and $R_{0}=$ $\mathbb{Q}\left[x_{1}, x_{2}, x_{3}, x_{4}, s_{1}, s_{2}\right]$. The ring $R$ is a free module of rank 2 over its subring $R_{0}$.

We have $C\left(\Gamma_{1}\right)=\{\mathbf{a}, \mathbf{b}\}\{-1\}$, where $\mathbf{a}=\left(u_{1}, u_{2}\right), \mathbf{b}=\left(x_{1}+x_{2}-\right.$ $\left.x_{3}-x_{4}, x_{1} x_{2}-x_{3} x_{4}\right)$, and $u_{1}=u_{1}\left(x_{1}, x_{2}, x_{3}, x_{4}\right), u_{2}=u_{2}\left(x_{1}, x_{2}, x_{3}, x_{4}\right)$. 
Hence $C(\Gamma)=\{\widetilde{\mathbf{a}}, \widetilde{\mathbf{b}}\}\{-2\}$ where

$$
(\widetilde{\mathbf{a}}, \widetilde{\mathbf{b}})=\left(\begin{array}{cc}
u_{1}^{\prime} & x_{1}+x_{2}-x_{5}-x_{6} \\
u_{2}^{\prime} & x_{1} x_{2}-x_{5} x_{6} \\
u_{1}^{\prime \prime} & x_{5}+x_{6}-x_{3}-x_{4} \\
u_{2}^{\prime \prime} & x_{5} x_{6}-x_{3} x_{4}
\end{array}\right)
$$

and

$$
\begin{array}{ll}
u_{1}^{\prime}=u_{1}\left(x_{1}, x_{2}, x_{5}, x_{6}\right), & u_{2}^{\prime}=u_{2}\left(x_{1}, x_{2}, x_{5}, x_{6}\right), \\
u_{1}^{\prime \prime}=u_{1}\left(x_{5}, x_{6}, x_{3}, x_{4}\right), & u_{2}^{\prime \prime}=u_{2}\left(x_{5}, x_{6}, x_{3}, x_{4}\right) .
\end{array}
$$

The first column of (22) lists entries of $\widetilde{\mathbf{a}}$, the second lists entries of $\widetilde{\mathbf{b}}$. All coefficients of $\widetilde{\mathbf{a}}$ and $\widetilde{\mathbf{b}}$ lie in $R_{0}$. Let $\{\widetilde{\mathbf{a}}, \widetilde{\mathbf{b}}\}_{0}$ be the restriction of $\{\widetilde{\mathbf{a}}, \widetilde{\mathbf{b}}\}$ to $R_{0}$, i.e., the tensor product of the factorizations

$$
R_{0} \stackrel{a_{i}}{\longrightarrow} R_{0} \stackrel{b_{i}}{\longrightarrow} R_{0}
$$

where $a_{i}, b_{i}$ are the entries of $\widetilde{\mathbf{a}}, \widetilde{\mathbf{b}}$. We can decompose

$$
\{\widetilde{\mathbf{a}}, \widetilde{\mathbf{b}}\} \cong\{\widetilde{\mathbf{a}}, \widetilde{\mathbf{b}}\}_{0} \oplus\{\widetilde{\mathbf{a}}, \widetilde{\mathbf{b}}\}_{0}\{2\}
$$

as an $\left(R_{0}, w\right)$-factorization, since $R \cong R_{0} \oplus R_{0}\{2\}$ as graded $R_{0}$-modules. The potential $w$ does not depend on the variables $s_{1}, s_{2}$ in $R_{0}$; the third entry of $\widetilde{\mathbf{b}}$ is $s_{1}-x_{3}-x_{4}$ and the fourth $s_{2}-x_{3} x_{4}$. Applying Proposition 10 to exclude $s_{1}$ and $s_{2}$, we conclude that $\{\widetilde{\mathbf{a}}, \widetilde{\mathbf{b}}\}_{0}$ is isomorphic in the category $\mathrm{hmf}_{w}$ to $\{\mathbf{a}, \mathbf{b}\}$. Proposition 30 follows.

Direct sum decomposition III. Consider the graphs $\Gamma, \Gamma_{1}, \Gamma_{2}$ of Figure 36.
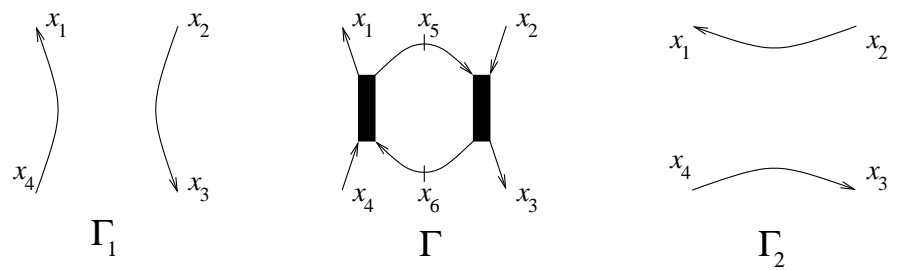

Fig. 36. Graphs $\Gamma_{1}, \Gamma, \Gamma_{2}$

$C(\Gamma), C\left(\Gamma_{1}\right), C\left(\Gamma_{2}\right)$ are homogeneous factorizations with the potential

$$
w=x_{1}^{n+1}-x_{2}^{n+1}+x_{3}^{n+1}-x_{4}^{n+1}
$$

over the ring $R^{\prime}=\mathbb{Q}\left[x_{1}, x_{2}, x_{3}, x_{4}\right]$.

Proposition 31. There is a direct sum decomposition in $\mathrm{hmf}_{w}$

$$
C(\Gamma) \cong C\left(\Gamma_{2}\right) \oplus\left(\bigoplus_{i=0}^{n-3} C\left(\Gamma_{1}\right)\langle 1\rangle\{3-n+2 i\}\right) .
$$


Proof. Define grading-preserving maps

$$
\alpha: C\left(\Gamma_{1}\right)\langle 1\rangle \rightarrow C(\Gamma)\{n-3\}, \quad \beta: C(\Gamma)\{n-3\} \rightarrow C\left(\Gamma_{1}\right)\langle 1\rangle
$$

as follows. $\alpha$ is the composition (see Figure 37)

$$
C\left(\Gamma_{1}\right)\langle 1\rangle \stackrel{\iota^{\prime}}{\rightarrow} C\left(\Gamma_{3}\right)\{n-1\} \stackrel{\chi_{0}^{\prime}}{\longrightarrow} C(\Gamma)\{n-3\},
$$

where $\iota$ is the tensor product of the identity of $C\left(\Gamma_{1}\right)$ with the "unit" map $\iota$ from $\mathbb{Q}\langle 1\rangle$ to the factorization assigned to a circle with two marks. $\chi_{0}^{\prime}$ is the composition of two $\chi_{0}$ maps.
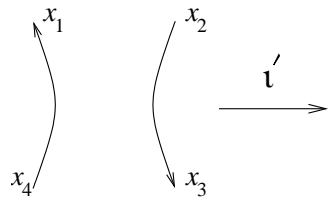

$\Gamma_{1}$

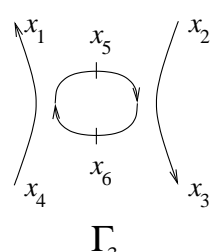

$\Gamma_{3}$

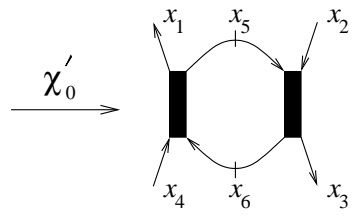

$\Gamma$

Fig. 37. Map $\alpha$

$\beta$ is the dual composition (see Figure 38)

$$
C(\Gamma) \stackrel{\chi_{1}^{\prime}}{\longrightarrow} C\left(\Gamma_{3}\right)\{-2\} \stackrel{\varepsilon^{\prime}}{\longrightarrow} C\left(\Gamma_{1}\right)\langle 1\rangle\{n-3\},
$$

where $\varepsilon^{\prime}$ is the tensor product of the identity of $C\left(\Gamma_{1}\right)$ with the trace map, and $\chi_{1}^{\prime}$ is the composition of two $\chi_{1}$ maps.

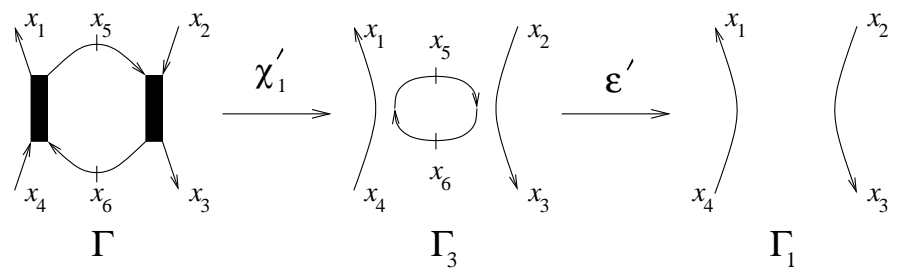

Fig. 38. Map $\beta$

Define grading-preserving maps $\alpha_{i}, \beta_{i}$ for $0 \leq i \leq n-3$ by

$$
\begin{aligned}
& \alpha_{i}: C\left(\Gamma_{1}\right)\langle 1\rangle\{3-n+2 i\} \rightarrow C(\Gamma), \quad \alpha_{i}=m\left(x_{5}^{i}\right) \alpha, \\
& \beta_{i}: C(\Gamma) \rightarrow C\left(\Gamma_{1}\right)\langle 1\rangle\{3-n+2 i\}, \quad \beta_{i}=\beta \sum_{a+b+c=n-3-i} m\left(x_{1}^{a} x_{3}^{b} x_{5}^{c}\right) .
\end{aligned}
$$

Lemma 3. $\beta_{j} \alpha_{i}=\delta_{i, j} \zeta$ Id.

Proof. A computation similar to the one in the proof of Lemma 1. We leave the details to the reader. 
Let $\alpha^{\prime}=\sum_{i=0}^{n-3} \alpha_{i}$ and $\beta^{\prime}=\zeta^{-1} \sum_{i=0}^{n-3} \beta_{i}$, considered as grading-preserving maps between

$$
N=\bigoplus_{i=0}^{n-3} C\left(\Gamma_{1}\right)\langle 1\rangle\{3-n+2 i\}
$$

and $C(\Gamma)$. Then $\beta^{\prime} \alpha^{\prime}=\operatorname{Id}_{N}$, so that $\alpha^{\prime} \beta^{\prime}$ is an idempotent endomorphism of $C(\Gamma)$ in the category $\mathrm{hmf}_{w}$. The splitting idempotents property in $\mathrm{hmf}_{w}$ (Proposition 24) implies that $C(\Gamma) \cong N \oplus M$ for some graded factorization $M$.

Lemma 4. $\operatorname{gdim} C(\Gamma)=s q^{-1}[n-1]\left(1+s q^{1-n}\right)\left(1+s q^{3-n}\right)$.

Proof. gdim $C(\Gamma)$ is the graded dimension of the cohomology of the 2complex $C(\Gamma) / \mathfrak{m} C(\Gamma)$, where $\mathfrak{m}$ is the maximal ideal $\left(x_{1}, x_{2}, x_{3}, x_{4}\right)$ of $R^{\prime}=$ $\mathbb{Q}\left[x_{1}, x_{2}, x_{3}, x_{4}\right]$. This quotient is a free module of rank 16 over the ring $R_{1}=\mathbb{Q}\left[x_{5}, x_{6}\right]$, and, after the shift by $\{2\}$, the cyclic Koszul complex (over $R_{1}$ ) of the pair

$$
(\mathbf{a}, \mathbf{b})=\left(\begin{array}{cc}
u_{1}^{\prime} & x_{5}-x_{6} \\
u_{2}^{\prime} & 0 \\
u_{1}^{\prime \prime} & x_{6}-x_{5} \\
u_{2}^{\prime \prime} & 0
\end{array}\right) \text {, }
$$

where

$$
\begin{array}{ll}
u_{1}^{\prime}=\left.u_{1}\left(x_{1}, x_{5}, x_{6}, x_{4}\right)\right|_{x_{1}=x_{4}=0}, & u_{1}^{\prime \prime}=\left.u_{1}\left(x_{3}, x_{6}, x_{5}, x_{2}\right)\right|_{x_{2}=x_{3}=0}, \\
u_{2}^{\prime}=\left.u_{2}\left(x_{1}, x_{5}, x_{6}, x_{4}\right)\right|_{x_{1}=x_{4}=0}, \quad u_{2}^{\prime \prime}=\left.u_{2}\left(x_{3}, x_{6}, x_{5}, x_{2}\right)\right|_{x_{2}=x_{3}=0} .
\end{array}
$$

We can modify this pair using transformations described at the end of Section 2 without changing its graded dimension. For instance, we can add the first entry of the second column to the third, simultaneously with subtracting the third entry of the first column from the first entry of the same column. This corresponds to a suitable change of basis in the free $R_{1}$-module $R_{1}^{\oplus 4}$. The resulting pair is

$$
\left(\begin{array}{cc}
u_{1}^{\prime}-u_{1}^{\prime \prime} & x_{5}-x_{6} \\
u_{2}^{\prime} & 0 \\
u_{1}^{\prime \prime} & 0 \\
u_{2}^{\prime \prime} & 0
\end{array}\right) .
$$

Moreover, $u_{1}^{\prime}-u_{1}^{\prime \prime}=0$, since $\{\mathbf{a}, \mathbf{b}\}$ is a 2 -complex, and $x_{5}-x_{6}$ is not a zero divisor. Using Proposition 9, we can cross out the first row of the above matrix and quotient the rest of the entries by the relation $x_{5}=x_{6}$ without changing the cohomology and graded dimension of this 2-complex. Thus, it 
suffices to find the graded dimension of the pair

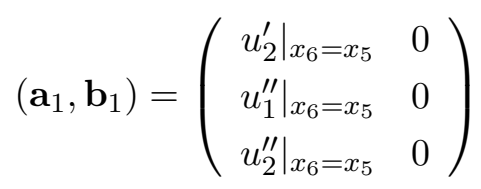

over the ring $\mathbb{Q}\left[x_{5}\right]$. A direct computation tells us that

$$
\begin{aligned}
& \left.u_{2}^{\prime}\right|_{x_{6}=x_{5}}=(n+1) x_{5}^{n}, \\
& \left.u_{1}^{\prime \prime}\right|_{x_{6}=x_{5}}=-(n+1) x_{5}^{n-1}, \\
& \left.u_{2}^{\prime \prime}\right|_{x_{6}=x_{5}}=-(n+1) x_{5}^{n-1} .
\end{aligned}
$$

Adding suitable multiples of the last entry of the first column to other entries of this column and dividing by $n+1$, we simplify the 2 -complex to

$$
\left(\begin{array}{cc}
0 & 0 \\
0 & 0 \\
x_{5}^{n-1} & 0
\end{array}\right) \text {. }
$$

The graded dimension of this 2-complex is the product of the graded dimensions of its rows (everything now is over the ring $\mathbb{Q}\left[x_{5}\right]$ ), since all rows but one consist of zeros. The entries $(n+1) x_{5}^{n}$ and $-(n+1) x_{5}^{n-1}$ have degrees $2 n$ and $2 n-2$, respectively. Therefore, the graded dimension of the first row is $1+s q^{1-n}$, while the graded dimension of the second row is $1+s q^{3-n}$. The graded dimension of $\left\{x_{5}^{n-1}, 0\right\}$ is $s\left(q^{n-1}+q^{n-3}+\cdots+q^{3-n}\right)=s q[n-1]$. The graded dimension of $C(\Gamma)$ is $q^{-2}$ times the product of these three graded dimensions. The lemma follows.

The graded dimension of $C\left(\Gamma_{1}\right)$ is the product of the graded dimensions of the factorizations assigned to its two arcs. Thus,

$$
\operatorname{gdim} C\left(\Gamma_{1}\right)=\left(1+s q^{1-n}\right)^{2} .
$$

Likewise,

$$
\operatorname{gdim} C\left(\Gamma_{2}\right)=\left(1+s q^{1-n}\right)^{2} .
$$

Recall that earlier we decomposed $C(\Gamma) \cong N \oplus M$. Therefore,

$$
\operatorname{gdim} C(\Gamma)=\operatorname{gdim} N+\operatorname{gdim} M .
$$

Clearly,

$$
\operatorname{gdim} N=s[n-2] \operatorname{gdim} C\left(\Gamma_{1}\right) .
$$

Lemma 5. $C\left(\Gamma_{2}\right)$ and $M$ have the same graded dimension.

Proof. This follows from Lemma 4 and the above equations. In the computation we use the fact that $s^{2}=1$.

Lemma 6. $\operatorname{gdim}_{\operatorname{Ext}} \operatorname{HMF}\left(C\left(\Gamma_{2}\right), C(\Gamma)\right)=q^{2 n-2}[2][n][n-1]$. 
The Ext groups here are computed in the category $\mathrm{HMF}_{w}$, and are naturally $\mathbb{Z} \oplus \mathbb{Z}_{2}$-graded, since the factorizations are homogeneous. The graded dimension gdim is the Poincaré polynomial of a $\mathbb{Z} \oplus \mathbb{Z}_{2}$-graded vector space.

Proof of Lemma 6. We start with the isomorphism

$$
\operatorname{Ext}_{\mathrm{HMF}}\left(C\left(\Gamma_{2}\right), C(\Gamma)\right) \cong H\left(C(\Gamma) \otimes_{R^{\prime}} C\left(\Gamma_{2}\right) \bullet\right)\{2 n-2\}
$$

of $\mathbb{Z} \oplus \mathbb{Z}_{2}$-graded vector spaces, implying the equality of the graded dimensions of the two sides. The 2-complex whose cohomology is computed on the right hand side (without the shift) is isomorphic to the 2-complex $C\left(\Gamma_{4}\right)$, where $\Gamma_{4}$, shown in Figure 39, is given by coupling $\Gamma_{2}$ to $\Gamma$.

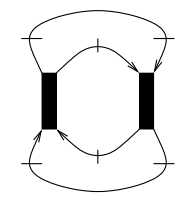

Fig. 39. Graph $\Gamma_{4}$

Applying Propositions 30 and 29, we find that $\operatorname{gdim} C\left(\Gamma_{4}\right)=[2][n][n-1]$. Lemma 6 follows.

Furthermore,

$$
\operatorname{gdim} \operatorname{Ext}_{\mathrm{HMF}}\left(C\left(\Gamma_{2}\right), C\left(\Gamma_{1}\right)\right)=q^{2 n-2}[n] s .
$$

Indeed, the left hand side, up to the shift $\{2 n-2\}$, is isomorphic to the cohomology of the factorization assigned to the graph given by coupling $\Gamma_{1}$ and $\Gamma_{2}$. This graph is a circle. The variable $s$ appears because Ext $^{1}{ }^{1}$ is nontrivial.

Since the factorization $N$ is the direct sum of $C\left(\Gamma_{1}\right)$ 's with shifts, we can compute the graded dimension of $\operatorname{Ext}_{\mathrm{HMF}}\left(C\left(\Gamma_{2}\right), N\right)$, and hence of the groups $\operatorname{Ext}_{\mathrm{HMF}}\left(C\left(\Gamma_{2}\right), M\right)$, as the difference of two graded dimensions. The result is

$$
\operatorname{gdim} \operatorname{Ext}_{\mathrm{HMF}}\left(C\left(\Gamma_{2}\right), M\right)=q^{2 n-2}[n]^{2} .
$$

Similarly, we compute that

$$
\operatorname{gdim}_{\operatorname{Ext}} \operatorname{HMF}\left(M, C\left(\Gamma_{2}\right)\right)=q^{2 n-2}[n]^{2} .
$$

To avoid the last computation, we could invoke Theorem 1 and its graded version (see [B, Example 10.1.6]) to show that the graded dimensions of the two Ext groups are equal.

Since $q^{2 n-2}[n]^{2} \in 1+q \mathbb{Z}[q]$, we obtain the following corollary.

Corollary 12. The vector space of morphisms between $C\left(\Gamma_{2}\right)$ and $M$ in $\mathrm{hmf}_{w}$ is isomorphic to $\mathbb{Q}$. 
In other words, any two nontrivial grading-preserving morphisms from $C\left(\Gamma_{2}\right)$ to $M$ are multiples of each other, and similarly for morphisms in the opposite direction.

After stripping off contractible summands from $M$ we obtain a graded factorization of rank 2 which has the form

$$
R^{\prime} \oplus R^{\prime}\{2-2 n\} \rightarrow R^{\prime}\{1-n\} \oplus R^{\prime}\{1-n\} \rightarrow R^{\prime} \oplus R^{\prime}\{2-2 n\} .
$$

Assume from now on that $M$ has been reduced to this minimal form. Then we strengthen the corollary, by noticing the absence of homotopies of degree $-1-n$ between $C\left(\Gamma_{2}\right)$ and $M$. We conclude that the space of gradingpreserving morphisms between $C\left(\Gamma_{2}\right)$ and $M$ in $\operatorname{mf}_{w}$ is one-dimensional (i.e. even before throwing out null-homotopic morphisms).

Let $\theta$ be a nontrivial grading-preserving morphism from $C\left(\Gamma_{2}\right)$ to $M$, and $\theta^{\prime}$ a nontrivial grading-preserving morphism from $M$ to $C\left(\Gamma_{2}\right)$. Each of these morphisms is unique up to scaling by a nonzero rational number.

Lemma 7. The composition $\theta^{\prime} \theta$ is nonzero.

Proof. Recall that a morphism between two factorizations can be described by a pair of matrices $\left(F_{0}, F_{1}\right)$ with polynomial coefficients. Define the rank of a morphism as the rank of $F_{0}$, treated as a matrix over the quotient field of the polynomial ring. The matrices $F_{0}$ and $F_{1}$ have the same rank.

The factorization $C\left(\Gamma_{2}\right)$ has the form

$$
\left(\begin{array}{c}
R \\
R\{2-2 n\}
\end{array}\right) \stackrel{P_{0}}{\longrightarrow}\left(\begin{array}{c}
R\{1-n\} \\
R\{1-n\}
\end{array}\right) \stackrel{P_{1}}{\longrightarrow}\left(\begin{array}{c}
R \\
R\{2-2 n\}
\end{array}\right),
$$

where

$$
P_{0}=\left(\begin{array}{cc}
\pi_{12} & x_{3}-x_{4} \\
\pi_{34} & x_{2}-x_{1}
\end{array}\right), \quad P_{1}=\left(\begin{array}{cc}
x_{1}-x_{2} & x_{3}-x_{4} \\
\pi_{34} & -\pi_{12}
\end{array}\right) .
$$

The factorization $M$ has the form

$$
\left(\begin{array}{c}
R \\
R\{2-2 n\}
\end{array}\right) \stackrel{V_{0}}{\longrightarrow}\left(\begin{array}{c}
R\{1-n\} \\
R\{1-n\}
\end{array}\right) \stackrel{V_{1}}{\longrightarrow}\left(\begin{array}{c}
R \\
R\{2-2 n\}
\end{array}\right),
$$

where $V_{0}, V_{1}$ are matrices with homogeneous entries (of degrees equal to the degrees of the matching entries in $\left.P_{1}, P_{2}\right)$ such that $V_{1} V_{0}=w \cdot$ Id.

Note that if $\theta^{\prime} \theta$ is homotopic to 0 , then it is actually zero, since there are no homotopies of degree $-n-1$ from $C\left(\Gamma_{2}\right)$ to itself. Assume that $\theta^{\prime} \theta=0$. This is only possible if the ranks of both $\theta^{\prime}$ and $\theta$ are equal to 1 . Let $\theta$ be given by a pair of matrices

$$
\Theta_{0}=\left(\begin{array}{ll}
f_{11} & f_{12} \\
f_{21} & f_{22}
\end{array}\right), \quad \Theta_{1}=\left(\begin{array}{ll}
g_{11} & g_{12} \\
g_{21} & g_{22}
\end{array}\right) .
$$


Since $\theta$ has zero degree, we see that all entries of $\Theta_{1}$ as well as the diagonal entries of $\Theta_{0}$ are rationals, $f_{12}=0$, and $f_{21}$ is a polynomial of degree $2 n-2$.

Assume that $f_{22} \neq 0$. Then we can rescale a basis vector of $M^{0}$ so that $f_{22}=1$. Since $\Theta_{0}$ has rank 1 , we see that $f_{11}=0$. By changing the basis in $M^{1}$ if necessary, we can assume $g_{21}=0$. Let

$$
V_{0}=\left(\begin{array}{ll}
v_{11} & v_{12} \\
v_{21} & v_{22}
\end{array}\right) .
$$

The equation $\Theta_{1} P_{0}=V_{0} \Theta_{0}$ implies

$$
v_{22} f_{21}=g_{22} \pi_{34}, \quad v_{22}=g_{22}\left(x_{2}-x_{1}\right) .
$$

This forces $g_{22}=0$, since $\pi_{34}$ is not divisible by $x_{2}-x_{1}$. Then $v_{22}=0$, which implies that $\operatorname{det}\left(V_{0}\right)=-v_{12} v_{21}$. This determinant must divide $w^{2}$ (which is the determinant of $\left.V_{1} V_{0}\right)$. This is impossible since $v_{12}$ is a homogeneous linear function of $x_{1}, x_{2}, x_{3}, x_{4}$ and

$$
w=x_{1}^{n+1}-x_{2}^{n+1}+x_{3}^{n+1}-x_{4}^{n+1} .
$$

Therefore, $f_{22}=0$. The equation $\Theta_{1} P_{0}=V_{0} \Theta_{0}$ implies

$$
g_{11}\left(x_{3}-x_{4}\right)+g_{12}\left(x_{2}-x_{1}\right)=0, \quad g_{21}\left(x_{3}-x_{4}\right)+g_{22}\left(x_{2}-x_{1}\right)=0 .
$$

Since $g_{i j}$ are rational numbers, they are all zeros. This is a contradiction, since $\Theta_{1}$ should have rank 1 .

The lemma implies that $\theta^{\prime} \theta$ is a nonzero multiple of the identity morphism of $C\left(\Gamma_{2}\right)$. In addition, we know that $C\left(\Gamma_{2}\right)$ and $M$ have the same graded dimension. Therefore, $C\left(\Gamma_{2}\right)$ and $M$ are isomorphic in $\mathrm{hmf}_{w}$. Proposition 31 follows.

Direct sum decomposition $I V$. Consider the graphs $\Gamma_{i}, 1 \leq i \leq 4$, depicted in Figure 40. The factorizations $C\left(\Gamma_{i}\right)$ have potential

$$
w=x_{1}^{n+1}+x_{2}^{n+1}+x_{3}^{n+1}-x_{4}^{n+1}-x_{5}^{n+1}-x_{6}^{n+1} .
$$

We view them as factorizations over the ring $R^{\prime}=\mathbb{Q}\left[x_{1}, \ldots, x_{6}\right]$.
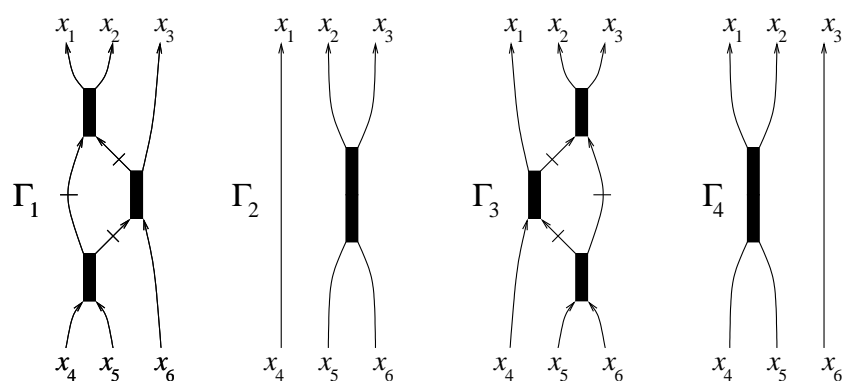

Fig. 40. Graphs $\Gamma_{1}, \Gamma_{2}, \Gamma_{3}, \Gamma_{4}$ 
Proposition 32. There is an isomorphism in $\mathrm{hmf}_{w}$

$$
C\left(\Gamma_{1}\right) \oplus C\left(\Gamma_{2}\right) \cong C\left(\Gamma_{3}\right) \oplus C\left(\Gamma_{4}\right) .
$$

Proof. If $n=1$, then $C\left(\Gamma_{i}\right)=0$ for all $i$, and the proposition is trivial. Assuming $n>1$, we introduce another factorization. Let

$$
\begin{array}{ll}
s_{1}=x_{1}+x_{2}+x_{3}, & s_{4}=x_{4}+x_{5}+x_{6}, \\
s_{2}=x_{1} x_{2}+x_{1} x_{3}+x_{2} x_{3}, & s_{5}=x_{4} x_{5}+x_{4} x_{6}+x_{5} x_{6}, \\
s_{3}=x_{1} x_{2} x_{3}, & s_{6}=x_{4} x_{5} x_{6} .
\end{array}
$$

Let $R_{s} \subset R^{\prime}$ be the subring generated by $s_{1}, \ldots, s_{6}$. It is isomorphic to the polynomial ring in $s_{1}, \ldots, s_{6}$. As an $R_{s}$-module, $R^{\prime}$ is free of rank 36 . Define a 3 -variable polynomial $h$ by

$$
h\left(s_{1}, s_{2}, s_{3}\right)=x_{1}^{n+1}+x_{2}^{n+1}+x_{3}^{n+1} .
$$

Then

$$
\begin{aligned}
w= & h\left(s_{1}, s_{2}, s_{3}\right)-h\left(s_{4}, s_{5}, s_{6}\right) \\
= & \frac{h\left(s_{1}, s_{2}, s_{3}\right)-h\left(s_{4}, s_{2}, s_{3}\right)}{s_{1}-s_{4}}\left(s_{1}-s_{4}\right) \\
& +\frac{h\left(s_{4}, s_{2}, s_{3}\right)-h\left(s_{4}, s_{5}, s_{3}\right)}{s_{2}-s_{5}}\left(s_{2}-s_{5}\right) \\
& \quad+\frac{h\left(s_{4}, s_{5}, s_{3}\right)-h\left(s_{4}, s_{5}, s_{6}\right)}{s_{3}-s_{6}}\left(s_{3}-s_{6}\right) \\
= & v_{1} \alpha_{1}+v_{2} \alpha_{2}+v_{3} \alpha_{3},
\end{aligned}
$$

where

$$
\begin{aligned}
\alpha_{1} & =s_{1}-s_{4}=x_{1}+x_{2}+x_{3}-x_{4}-x_{5}-x_{6}, \\
\alpha_{2} & =s_{2}-s_{5}=x_{1} x_{2}+x_{1} x_{3}+x_{2} x_{3}-x_{4} x_{5}-x_{4} x_{6}-x_{5} x_{6}, \\
\alpha_{3} & =s_{3}-s_{6}=x_{1} x_{2} x_{3}-x_{4} x_{5} x_{6}, \\
v_{1} & =\frac{h\left(s_{1}, s_{2}, s_{3}\right)-h\left(s_{4}, s_{2}, s_{3}\right)}{s_{1}-s_{4}}, \\
v_{2} & =\frac{h\left(s_{4}, s_{2}, s_{3}\right)-h\left(s_{4}, s_{5}, s_{3}\right)}{s_{2}-s_{5}}, \\
v_{3} & =\frac{h\left(s_{4}, s_{5}, s_{3}\right)-h\left(s_{4}, s_{5}, s_{6}\right)}{s_{3}-s_{6}} .
\end{aligned}
$$

$v_{1}, v_{2}, v_{3}$ are polynomials in $x_{1}, \ldots, x_{6}$. The quotient of $R_{s}$ by the ideal $\left(\alpha_{1}, \alpha_{2}, \alpha_{3}\right)$ is isomorphic to $\mathbb{Q}\left[s_{1}, s_{2}, s_{3}\right]$. The images of $v_{1}, v_{2}, v_{3}$ in this quotient ring have the form

$$
\frac{\partial h\left(s_{1}, s_{2}, s_{3}\right)}{\partial s_{1}}, \quad \frac{\partial h\left(s_{1}, s_{2}, s_{3}\right)}{\partial s_{2}}, \quad \frac{\partial h\left(s_{1}, s_{2}, s_{3}\right)}{\partial s_{3}} .
$$


It is well-known that the quotient of $\mathbb{Q}\left[s_{1}, s_{2}, s_{3}\right]$ by the ideal generated by these derivatives is isomorphic to the cohomology ring of the Grassmannian of 3-dimensional subspaces of $\mathbb{C}^{n}$ (see [MC, p. 127], for example). Thus, there is an algebra isomorphism

$$
R_{s} /\left(\alpha_{1}, \alpha_{2}, \alpha_{3}, v_{1}, v_{2}, v_{3}\right) \cong \mathrm{H}^{*}(\operatorname{Gr}(3, n), \mathbb{Q}) .
$$

In the degenerate case $n=2$ both sides are zero.

Likewise, the quotient ring $R^{\prime} /\left(\alpha_{1}, \alpha_{2}, \alpha_{3}, v_{1}, v_{2}, v_{3}\right)$ is the cohomology ring of the configuration space

$$
\left\{N_{1} \subset N_{2} \subset N_{3} \supset N_{2}^{\prime} \supset N_{1}^{\prime} \mid \operatorname{dim} N_{i}=\operatorname{dim} N_{i}^{\prime}=i, N_{i}, N_{i}^{\prime} \subset \mathbb{C}^{n}\right\} .
$$

Lemma 8. The sequence $\left(\alpha_{1}, \alpha_{2}, \alpha_{3}, v_{1}, v_{2}, v_{3}\right)$ and each of its permutations are regular sequences in the rings $R_{s}$ and $R^{\prime}$.

Proof. The length of the sequence equals the number of generators of the polynomial rings $R_{s}$ and $R^{\prime}$. Since the quotient rings by the ideal generated by this sequence are finite-dimensional, the sequence is regular.

Let $\Upsilon$ be the following $\left(R^{\prime}, w\right)$-factorization:

$$
\Upsilon:=\left\{\left(v_{1}, v_{2}, v_{3}\right),\left(\alpha_{1}, \alpha_{2}, \alpha_{3}\right)\right\}\{-3\} .
$$

$\Upsilon$ is the tensor product of the factorizations

$$
\begin{aligned}
& R^{\prime} \stackrel{v_{1}}{\longrightarrow} R^{\prime}\{1-n\} \stackrel{\alpha_{1}}{\longrightarrow} R^{\prime}, \\
& R^{\prime} \stackrel{v_{2}}{\longrightarrow} R^{\prime}\{3-n\} \stackrel{\alpha_{2}}{\longrightarrow} R^{\prime}, \\
& R^{\prime} \stackrel{v_{3}}{\longrightarrow} R^{\prime}\{5-n\} \stackrel{\alpha_{3}}{\longrightarrow} R^{\prime},
\end{aligned}
$$

shifted by $\{-3\}$. One could think of $\Upsilon$ as the factorization assigned to the diagram in the lower left corner of Figure 4.

Proposition 32 will follow from

Proposition 33. There is an isomorphism in $\mathrm{hmf}_{w}$

$$
C\left(\Gamma_{1}\right) \cong \Upsilon \oplus C\left(\Gamma_{4}\right) .
$$

Indeed, the factorization $C\left(\Gamma_{1}\right)$ turns into $C\left(\Gamma_{3}\right)$, and $C\left(\Gamma_{2}\right)$ into $C\left(\Gamma_{4}\right)$ if we permute $x_{1}$ with $x_{3}$ and $x_{4}$ with $x_{6}$. The factorization $\Upsilon$ is invariant under this operation, since $\alpha_{i}$ 's and $v_{i}$ 's are, so that Proposition 33 also implies the isomorphism

$$
C\left(\Gamma_{3}\right) \cong \Upsilon \oplus C\left(\Gamma_{2}\right) .
$$

Then both sides in the equation of Proposition 32 are isomorphic to $\Upsilon \oplus$ $C\left(\Gamma_{2}\right) \oplus C\left(\Gamma_{4}\right)$. Thus, it suffices to establish Proposition 33.

The proof of Proposition 33 occupies the next 10 pages.

Lemma 9. $C\left(\Gamma_{4}\right)$ is a direct summand of $C\left(\Gamma_{1}\right)$. 
Proof. Let

$$
g_{0}=\chi_{0} r_{0}, \quad C\left(\Gamma_{4}\right) \stackrel{r_{0}}{\longrightarrow} C\left(\Gamma_{5}\right) \stackrel{\chi_{0}}{\longrightarrow} C\left(\Gamma_{1}\right)
$$

be the composition depicted in Figure 41.

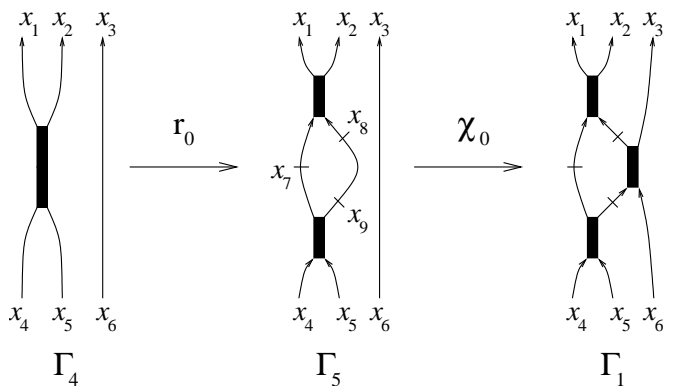

Fig. 41. Map $g_{0}$

Proposition 30 implies that $C\left(\Gamma_{5}\right) \cong C\left(\Gamma_{4}\right)\{-1\} \oplus C\left(\Gamma_{4}\right)\{1\}$, and $r_{0}$ is defined to be the inclusion of $C\left(\Gamma_{4}\right)$ into $C\left(\Gamma_{5}\right)$ as the direct summand $C\left(\Gamma_{4}\right)\{-1\}$. The maps $r_{0}$ and $\chi_{0}$ have degrees -1 and 1 , respectively, so that $g_{0}$ is grading-preserving.

Let

$$
g_{1}=r_{1} \chi_{1}, \quad C\left(\Gamma_{1}\right) \stackrel{\chi_{1}}{\longrightarrow} C\left(\Gamma_{5}\right) \stackrel{r_{1}}{\longrightarrow} C\left(\Gamma_{4}\right)
$$

be the composition depicted in Figure 42.

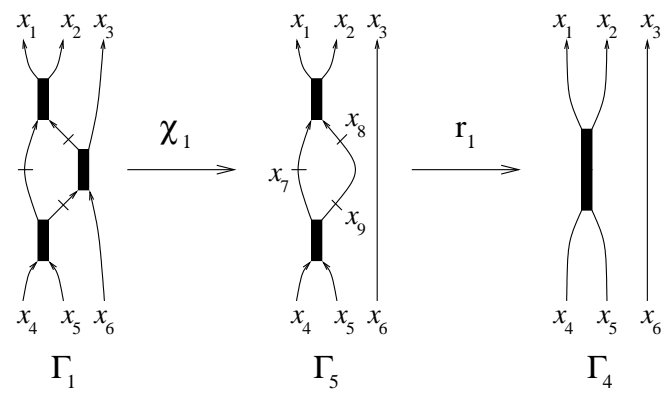

Fig. 42. Map $g_{1}$

$r_{1}$ is the projection of $C\left(\Gamma_{5}\right) \cong C\left(\Gamma_{4}\right)\{-1\} \oplus C\left(\Gamma_{4}\right)\{1\}$ onto the second direct summand (the direct sum decomposition is the one obtained in the proof of Proposition 30).

We compute

$$
g_{1} g_{0}=r_{1} \chi_{1} \chi_{0} r_{0}=r_{1} m\left(x_{8}-x_{6}\right) r_{0}=r_{1} m\left(x_{8}\right) r_{0},
$$

since $r_{1} m\left(x_{6}\right) r_{0}=0$. The proof of Proposition 30 implies that $r_{1} m\left(x_{8}\right) r_{0}$ is a nonzero multiple of the identity morphism. Therefore, $g_{0}$ is split injective, and $C\left(\Gamma_{4}\right)$ is a direct summand of $C\left(\Gamma_{1}\right)$. 
Let $M$ be the complement of $C\left(\Gamma_{4}\right)$ in $C\left(\Gamma_{1}\right)$,

$$
C\left(\Gamma_{1}\right) \cong M \oplus C\left(\Gamma_{4}\right) .
$$

Lemma 10. $\operatorname{gdim} C\left(\Gamma_{1}\right)=q^{-3}\left(1+q^{2}\right)\left(1+s q^{1-n}\right)\left(1+s q^{3-n}\right)^{2}$ if $n>2$.

Proof. Similar to that of Lemma 4. Up to a shift, $C\left(\Gamma_{1}\right)$ is the Koszul factorization $\{\mathbf{a}, \mathbf{b}\}$ for some length 6 sequences whose entries are polynomials in $x_{1}, \ldots, x_{6}$ and in three other variables (say $y_{1}, y_{2}, y_{3}$ ) assigned to the three internal marks of $\Gamma_{1}$. We can exclude two of these variables using Proposition 9, and then specialize to $x_{1}=\cdots=x_{6}=0$, since we are computing cohomology. The details are left to the reader.

In particular, if $n>2$, the dimension of $C\left(\Gamma_{1}\right)$ is 16 . If $n=2$, the factorization $C\left(\Gamma_{1}\right)$ has dimension 8, which is also the dimension of $C\left(\Gamma_{4}\right)$. Hence, $M=0$ if $n=2$, and there is an isomorphism $C\left(\Gamma_{1}\right) \cong C\left(\Gamma_{4}\right)$. The factorization $\Upsilon$ is contractible $\left(v_{3}=3\right.$ if $\left.n=2\right)$, and Propositions 33, 32 follow. From now on we assume $n>2$.

It is easy to see that

$$
\begin{aligned}
\operatorname{gdim} C\left(\Gamma_{4}\right) & =q^{-1}\left(1+s q^{1-n}\right)^{2}\left(1+s q^{3-n}\right), \\
\operatorname{gdim} \Upsilon & =q^{-3}\left(1+s q^{1-n}\right)\left(1+s q^{3-n}\right)\left(1+s q^{5-n}\right) .
\end{aligned}
$$

Therefore,

$$
\operatorname{gdim} M=\operatorname{gdim} C\left(\Gamma_{1}\right)-\operatorname{gdim} C\left(\Gamma_{4}\right)=\operatorname{gdim} \Upsilon .
$$

Thus, $M$ and $\Upsilon$ have the same graded dimension.

LEMMA 11.

$$
\operatorname{gdim} \operatorname{Ext}\left(\Upsilon, C\left(\Gamma_{4}\right)\right)=q^{3 n-3}[2][n][n-1][n-2] .
$$

Proof. The Ext groups in the lemma are isomorphic to the cohomology of the 2-complex

$$
C\left(\Gamma_{4}\right) \otimes \Upsilon_{\bullet} \cong\{\mathbf{a}, \mathbf{b}\}\langle 1\rangle\{3 n-9\},
$$

where

$$
(\mathbf{a}, \mathbf{b})=\left(\begin{array}{cc}
\pi_{36} & x_{3}-x_{6} \\
u_{1}\left(x_{1}, x_{2}, x_{4}, x_{5}\right) & x_{1}+x_{2}-x_{4}-x_{5} \\
u_{2}\left(x_{1}, x_{2}, x_{4}, x_{5}\right) & x_{1} x_{2}-x_{4} x_{5} \\
\alpha_{1} & v_{1} \\
\alpha_{2} & v_{2} \\
\alpha_{3} & v_{3}
\end{array}\right)
$$

Pass to the subring $R^{\prime \prime}$ of $R^{\prime}=\mathbb{Q}\left[x_{1}, \ldots, x_{6}\right]$ generated by

$$
x_{1}^{\prime}=x_{1}+x_{2}, \quad x_{2}^{\prime}=x_{1} x_{2}, \quad x_{3}, \quad x_{4}^{\prime}=x_{4}+x_{5}, \quad x_{5}^{\prime}=x_{4} x_{5}, \quad x_{6},
$$


and denote by $\{\mathbf{a}, \mathbf{b}\}_{0}$ the cyclic Koszul complex of the pair $(\mathbf{a}, \mathbf{b})$ viewed as a pair in $R^{\prime \prime}$ (this is possible since all coefficients of $\mathbf{a}$ and $\mathbf{b}$ lie in $R^{\prime \prime}$ ). Then

$$
\operatorname{gdim} H(\{\mathbf{a}, \mathbf{b}\})=q^{2}[2]^{2} \operatorname{gdim} H\left(\{\mathbf{a}, \mathbf{b}\}_{0}\right) .
$$

We use the first three lines of $(\mathbf{a}, \mathbf{b})_{0}$ to exclude $x_{6}, x_{4}^{\prime}$, and $x_{5}^{\prime}$, and denote by $\left(\mathbf{a}^{1}, \mathbf{b}^{1}\right)$ the pair obtained by crossing out the first three lines of $(\mathbf{a}, \mathbf{b})_{0}$ and passing to the quotient ring of $R^{\prime \prime}$ by the relations $x_{6}=x_{4}^{\prime}=x_{5}^{\prime}=0$. Then $\mathbf{a}^{1}=(0,0,0), \mathbf{b}^{1}=\left(-v_{1},-v_{2},-v_{3}\right)$,

$$
H\left(\{\mathbf{a}, \mathbf{b}\}_{0}\right) \cong H\left(\left\{\mathbf{a}^{1}, \mathbf{b}^{1}\right\}\right) \cong H\left((0,0,0),\left(v_{1}, v_{2}, v_{3}\right)\right),
$$

and the lemma follows easily, since $\left(v_{1}, v_{2}, v_{3}\right)$ is a regular sequence in the quotient ring $R^{\prime \prime \prime}=R^{\prime \prime} /\left(x_{6}, x_{4}^{\prime}, x_{5}^{\prime}\right)$, and $R^{\prime \prime \prime} /\left(v_{1}, v_{2}, v_{3}\right)$ is isomorphic to the cohomology ring of the partial flag variety

$$
\left\{N_{2} \subset N_{3} \mid \operatorname{dim}\left(N_{i}\right)=i, N_{3} \subset \mathbb{C}^{n}\right\} .
$$

LEMMA 12.

$$
\operatorname{gdim} \operatorname{Ext}\left(\Upsilon, C\left(\Gamma_{1}\right)\right)=q^{3 n-3}[2]^{3}[n][n-1][n-2] .
$$

Proof. The Ext groups in the lemma are isomorphic to the cohomology of the 2-complex $C\left(\Gamma_{1}\right) \otimes \Upsilon_{\bullet}$. This is a complex of free $R^{1}$-modules, where $R^{1}$ is the ring of polynomials in $x_{1}, \ldots, x_{6}$ and three "internal" variables corresponding to the three marks in $\Gamma_{1}$. Passing to a suitable subring (over which $R^{1}$ is a free rank 4 module) and excluding several variables, one can show that $C\left(\Gamma_{1}\right) \otimes \Upsilon_{\bullet}$ is isomorphic (up to contractible complexes) to the direct sum of four copies of $C\left(\Gamma_{1}\right) \otimes \Upsilon_{\bullet}$ with shifts, implying

$$
\operatorname{gdim} \operatorname{Ext}\left(\Upsilon, C\left(\Gamma_{1}\right)\right)=[2]^{2} \operatorname{gdim} \operatorname{Ext}\left(\Upsilon, C\left(\Gamma_{4}\right)\right) .
$$

The lemmas imply

$$
\begin{aligned}
\operatorname{gdim} \operatorname{Ext}(\Upsilon, M) & =\operatorname{gdim} \operatorname{Ext}\left(\Upsilon, C\left(\Gamma_{1}\right)\right)-\operatorname{gdim} \operatorname{Ext}\left(\Upsilon, C\left(\Gamma_{4}\right)\right) \\
& =q^{3 n-3}[2][3][n][n-1][n-2] .
\end{aligned}
$$

Since $q^{3 n-3}[2][3][n][n-1][n-2] \in 1+q \mathbb{Z}[q]$, the space of degree 0 morphisms from $\Upsilon$ to $M$ is one-dimensional. Let $\theta$ be a nontrivial (not null-homotopic) degree 0 morphism from $\Upsilon$ to $M$.

LEMMA 13. $\theta$ is an isomorphism of factorizations.

Proof. The factorization $\Upsilon$ has the form

$$
\left(\begin{array}{c}
R^{\prime}\{-3\} \\
R^{\prime}\{5-2 n\} \\
R^{\prime}\{3-2 n\} \\
R^{\prime}\{1-2 n\}
\end{array}\right) \stackrel{\Lambda_{0}}{\longrightarrow}\left(\begin{array}{c}
R^{\prime}\{2-n\} \\
R^{\prime}\{-n\} \\
R^{\prime}\{-2-n\} \\
R^{\prime}\{6-3 n\}
\end{array}\right) \stackrel{\Lambda_{1}}{\longrightarrow}\left(\begin{array}{c}
R^{\prime}\{-3\} \\
R^{\prime}\{5-2 n\} \\
R^{\prime}\{3-2 n\} \\
R^{\prime}\{1-2 n\}
\end{array}\right),
$$


where

$$
\Lambda_{0}=\left(\begin{array}{cccc}
v_{3} & -\alpha_{2} & -\alpha_{1} & 0 \\
v_{2} & \alpha_{3} & 0 & -\alpha_{1} \\
v_{1} & 0 & \alpha_{3} & \alpha_{2} \\
0 & v_{1} & -v_{2} & v_{3}
\end{array}\right), \quad \Lambda_{1}=\left(\begin{array}{cccc}
\alpha_{3} & \alpha_{2} & \alpha_{1} & 0 \\
-v_{2} & v_{3} & 0 & \alpha_{1} \\
-v_{1} & 0 & v_{3} & -\alpha_{2} \\
0 & -v_{1} & v_{2} & \alpha_{3}
\end{array}\right) .
$$

We can assume that the factorization $M=M^{0} \oplus M^{1}$ has no contractible summands. Since it has the same graded dimension as $\Upsilon$, we can write $M$ as

$$
\left(\begin{array}{c}
R^{\prime}\{-3\} \\
R^{\prime}\{5-2 n\} \\
R^{\prime}\{3-2 n\} \\
R^{\prime}\{1-2 n\}
\end{array}\right) \stackrel{\Pi_{0}}{\longrightarrow}\left(\begin{array}{c}
R^{\prime}\{2-n\} \\
R^{\prime}\{-n\} \\
R^{\prime}\{-2-n\} \\
R^{\prime}\{6-3 n\}
\end{array}\right) \stackrel{\Pi_{1}}{\longrightarrow}\left(\begin{array}{c}
R^{\prime}\{-3\} \\
R^{\prime}\{5-2 n\} \\
R^{\prime}\{3-2 n\} \\
R^{\prime}\{1-2 n\}
\end{array}\right)
$$

for some $4 \times 4$ matrices $\Pi_{0}, \Pi_{1}$ with homogeneous entries whose degrees match the degrees of the entries of $\Lambda_{0}, \Lambda_{1}$. Let $\Pi_{0}=\left(a_{i j}\right)_{i, j=1}^{4}$ and $\Pi_{1}=$ $\left(b_{i j}\right)_{i, j=1}^{4}$. The entry $a_{14}$ is a degree $n+1$ homomorphism from $R^{\prime}\{1-2 n\}$ to $R^{\prime}\{2-n\}$, and necessarily a rational number. If $a_{14} \neq 0$, then $M$ contains a contractible summand, which is a contradiction. Therefore, $a_{14}=0$. Likewise, $b_{14}=0$ if $n>4$.

We can write the morphism $\theta$ via a pair of matrices

$$
\Theta_{0}=\left(c_{i j}\right)_{i, j=1}^{4}, \quad \Theta_{1}=\left(f_{i j}\right)_{i, j=1}^{4} .
$$

Since $\theta$ is a morphism of factorizations, the diagram

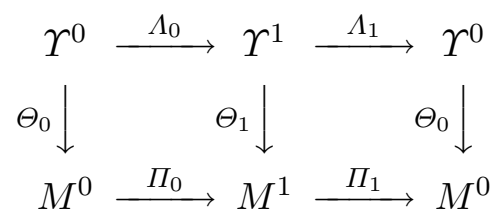

is commutative, and the following relations hold:

$$
\begin{aligned}
& \Pi_{0} \Theta_{0}=\Theta_{1} \Lambda_{0}, \\
& \Pi_{1} \Theta_{1}=\Theta_{0} \Lambda_{1} .
\end{aligned}
$$

$\theta$ is grading-preserving. If $n>4$, then the sequences of degree shifts $(-3,5-$ $2 n, 3-2 n, 1-2 n)$ and $(2-n,-n,-2-n, 6-3 n)$ are strictly decreasing, and the matrices $\Theta_{0}, \Theta_{1}$ are lower-triangular, $c_{i j}=f_{i j}=0$ for $i<j$, since there are no grading-preserving homomorphisms from $R^{\prime}\left\{k_{1}\right\}$ to $R^{\prime}\left\{k_{2}\right\}$ if $k_{2}>k_{1}$. If $n=4$, the sequences are not strictly decreasing, but changing bases in $M^{0}$ and $M^{1}$ if necessary, we can assume that $\Theta_{0}$ and $\Theta_{1}$ are lower-triangular in this case too. When $n=3$, we cannot assume right away that $\Theta_{0}$ and $\Theta_{1}$ are lower-triangular. We postpone considering the cases $n=3$ and $n=4$, 
and from now on until two paragraphs after the proof of Lemma 18 restrict to the case $n>4$.

$\theta$ is an isomorphism iff both $\Theta_{0}$ and $\Theta_{1}$ are invertible over the ring $R^{\prime}$. Since they are lower-triangular with rational diagonal entries, they are invertible iff all diagonal entries are nonzero. Moreover, $\Theta_{1}$ is invertible iff $\Theta_{0}$ is invertible. This is due to the equation $\Pi_{0} \Theta_{0} \Lambda_{0}^{-1}=\Theta_{1}$ and the fact that the determinants of $\Pi_{0}$ and $\Lambda_{0}$ are nonzero multiples of $w^{2}$ (to verify this property, use the fact that $w$ is an irreducible polynomial, $\Lambda_{0} \Lambda_{1}=\Pi_{0} \Pi_{1}=w \mathrm{I}$, and $\Pi_{0}$ has coefficients in the same degrees as $\Lambda_{0}$ ).

Thus, $\theta$ is an isomorphism iff $\Theta_{0}$ is invertible.

The matrix equation (27) can be rewritten as 16 equations for the entries of the matrices $\Pi_{0}, \Theta_{0}, \Theta_{1}$. Three of these equations have the form

$$
\begin{aligned}
& a_{24} c_{44}=-\alpha_{1} f_{22}, \\
& a_{34} c_{44}=-\alpha_{1} f_{32}+\alpha_{2} f_{33}, \\
& a_{44} c_{44}=-\alpha_{1} f_{42}+\alpha_{2} f_{43}+v_{3} f_{44} .
\end{aligned}
$$

Lemma 14. $c_{44} \neq 0$.

Proof. Assume $c_{44}=0$. Then the above equations reduce to

$$
\begin{aligned}
& 0=-\alpha_{1} f_{22}, \\
& 0=-\alpha_{1} f_{32}+\alpha_{2} f_{33}, \\
& 0=-\alpha_{1} f_{42}+\alpha_{2} f_{43}+v_{3} f_{44} .
\end{aligned}
$$

Since $f_{22}, f_{33}, f_{44} \in \mathbb{Q}$, and the sequence $\left(\alpha_{1}, \alpha_{2}, v_{3}\right)$ is regular, the equations imply

$$
f_{22}=f_{32}=f_{33}=f_{44}=0 .
$$

The last equation of the three above simplifies to

$$
f_{42} \alpha_{1}=f_{43} \alpha_{2} .
$$

Since $\alpha_{1}$ and $\alpha_{2}$ are relatively prime,

$$
f_{42}=\alpha_{2} z_{0}, \quad f_{43}=\alpha_{1} z_{0},
$$

for some polynomial $z_{0}$.

Three of the equations in (28) are

$$
\begin{aligned}
& b_{24} f_{44}=\alpha_{1} c_{22}, \\
& b_{34} f_{44}=\alpha_{1} c_{32}-\alpha_{2} c_{33}, \\
& b_{44} f_{44}=\alpha_{1} c_{42}-\alpha_{2} c_{43}+\alpha_{3} c_{44} .
\end{aligned}
$$

Since $f_{44}=0$, and $\left(\alpha_{1}, \alpha_{2}, \alpha_{3}\right)$ is a regular sequence, we derive

and

$$
c_{22}=c_{32}=c_{33}=c_{44}=0,
$$

$$
c_{42}=\alpha_{2} z_{1}, \quad c_{43}=\alpha_{1} z_{1},
$$

for some polynomial $z_{1}$. 
The remaining terms of the matrix equations (27), (28) imply

$$
\begin{array}{lll}
f_{21}=-a_{24} z_{1}, & f_{31}=-a_{34} z_{1}, & f_{41}=\alpha_{3} z_{0}-a_{44} z_{1}, \\
c_{21}=b_{24} z_{0}, & c_{31}=b_{34} z_{0}, & c_{41}=b_{44} z_{0}-v_{3} z_{1} .
\end{array}
$$

Let $Z_{0}, Z_{1}$ be $4 \times 4$ matrices with the only nonzero entry $z_{0}$ (respectively, $z_{1}$ ) at the intersection of the fourth row and the first column. Then

$$
\Theta_{0}=\Pi_{1} Z_{0}-Z_{1} \Lambda_{0}, \quad \Theta_{1}=Z_{0} \Lambda_{1}-\Pi_{0} Z_{1} .
$$

We see that $\theta$ is homotopic to 0 through the homotopy $\left(Z_{0},-Z_{1}\right)$. This contradicts our assumption on $\theta$. Therefore, $c_{44} \neq 0$, and Lemma 14 is proved.

LEMMA $15 . f_{44} \neq 0$.

Proof. An argument in the proof of the previous lemma shows that $f_{44}$ $=0$ implies $c_{44}=0$.

Since $f_{44} \neq 0$ and $c_{44} \neq 0$, by rescaling the last basis vector in $M^{0}$ and in $M^{1}$, we can assume

$$
f_{44}=c_{44}=1 .
$$

To finish the proof of Lemma 13 in the case $n>3$, we assume to the contrary that $\theta$ is not an isomorphism.

LEMMA 16. $f_{11}=0$ if $\theta$ is not an isomorphism.

Proof. Suppose $f_{11} \neq 0$. By changing the first basis vector in $M^{1}$ if necessary, we can reduce to the case

$$
f_{11}=1, \quad f_{21}=f_{31}=f_{41}=0 .
$$

Entry $(1,3)$ of equation $(27)$ is

$$
a_{13} c_{33}=-\alpha_{1} f_{11}=-\alpha_{1} .
$$

Therefore $c_{33} \neq 0$, and we assume, without loss of generality (by changing the third basis vector in $M^{0}$ ), that

$$
c_{33}=1, \quad c_{43}=0 .
$$

Then $a_{13}=-\alpha_{1}$, and equation $(27)$, entry $(1,2)$, simplifies to

$$
-\alpha_{2}=a_{12} c_{22}-\alpha_{1} c_{32} \text {. }
$$

Since $\alpha_{2}$ is not divisible by $\alpha_{1}$, we know that $c_{22} \neq 0$. Changing the second basis vector in $M^{0}$, we assume

$$
c_{22}=1, \quad c_{32}=0, \quad c_{42}=0, \quad a_{12}=-\alpha_{2} .
$$

Since $c_{22}=c_{33}=c_{44}=1$ and $\Theta_{0}$ is not invertible (as we assumed that $\theta$ is not an isomorphism), necessarily $c_{11}=0$. 
Entry $(1,1)$ of $(27)$ can be written as

$$
v_{3}=-\alpha_{2} c_{21}-\alpha_{1} c_{31} \text {. }
$$

Contradiction, since $v_{3}$ is not in the ideal $\left(\alpha_{1}, \alpha_{2}\right)$. Lemma 16 follows.

LEMMA 17. $c_{11}=0$ if $\theta$ is not an isomorphism.

Proof. Suppose otherwise $\left(c_{11} \neq 0\right)$ and change the first basis vector of $M^{0}$ so that

$$
c_{11}=1, \quad c_{21}=c_{31}=c_{41}=0 .
$$

From the previous lemma we know that $f_{11}=0$. Entry $(1,3)$ of $(28)$ reads $\alpha_{1}=b_{13} f_{33}$. We see that $f_{33} \neq 0$, and after modifying the third basis vector of $M^{1}$ we assume

$$
f_{33}=1, \quad f_{43}=0, \quad b_{13}=\alpha_{1} .
$$

Entry $(1,2)$ of the equation $(28)$ reads

$$
\alpha_{2}=b_{12} f_{22}+\alpha_{1} f_{32} .
$$

Since $\alpha_{2}$ does not factor, $f_{22} \neq 0$, and we can reduce to the case

$$
f_{22}=1, \quad f_{32}=f_{42}=0, \quad b_{12}=\alpha_{2} .
$$

Entry $(1,1)$ of $(28)$ simplifies to

$$
\alpha_{3}=\alpha_{2} f_{21}+\alpha_{1} f_{31} .
$$

Contradiction, since $\alpha_{3}$ is not in the ideal generated by $\alpha_{1}$ and $\alpha_{2}$. Lemma 17 follows.

By now, we have reduced our considerations to the case

$$
f_{44}=c_{44}=1, \quad f_{11}=c_{11}=0 .
$$

LEMMA 18. $f_{33}=0$ if $\theta$ is not an isomorphism.

Proof. If $f_{33} \neq 0$, we can assume $f_{33}=1, f_{43}=0$. Entry $(1,3)$ of equation (28) becomes $b_{13}=0$. Note that $b_{12} \neq 0$; otherwise $b_{11}$ (which is a polynomial of degree 3 in $x_{i}$ 's) would be the only nonzero entry in the first row of $\Pi_{1}$, and a divisor of $\operatorname{det} \Pi_{1}$, which is proportional to $w^{2}$. Since $w$ is irreducible, this is impossible, so that $b_{12} \neq 0$.

Entry $(1,2)$ of $(28)$ simplifies to $b_{12} f_{22}=0$. Thus, $f_{22}=0$. Entry $(1,1)$ of the same equation reduces to $b_{12} f_{21}=0$. Thus, $f_{21}=0$.

Next, entries $(2,3)$ and $(2,4)$ of $(28)$ reduce to

$$
b_{24}=\alpha_{1} c_{22}, \quad b_{23}=\alpha_{1} c_{21} .
$$

Entry $(2,2)$ simplifies to

$$
b_{23} f_{32}+b_{24} f_{42}=\alpha_{2} c_{21}+v_{3} c_{22} .
$$

Since $v_{3}$ is not in the ideal generated by $\alpha_{1}, \alpha_{2}$, we know that $c_{22}=0$. Then $b_{24}=\alpha_{1} c_{22}=0($ entry $(2,4)$ of $(28))$. Entry $(2,3)$ tells us that $b_{23}=\alpha_{1} c_{21}$, 
while entry $(2,2)$ reduces to $\alpha_{2} c_{21}=\alpha_{1} c_{21} f_{32}$. Since $\alpha_{2}$ is not divisible by $\alpha_{1}$, we have $c_{21}=0$ and $b_{23}=0$.

Switching to equation $(27)$, and looking at entry $(4,4)$, we get $a_{44}=$ $v_{3}-\alpha_{1} f_{42}$, while entry $(4,3)$ simplifies to

$$
-v_{2}-\alpha_{1} f_{41}=a_{43} c_{33}+\left(v_{3}-\alpha_{1} f_{42}\right) c_{43} \text {. }
$$

Since $v_{2}$ does not lie in the ideal $\left(\alpha_{1}, v_{3}\right)$, we have $c_{33} \neq 0$. Then we can assume $c_{33}=1, c_{43}=0$. Entries $(1,3)$ and $(2,3)$ tell us that $a_{13}=a_{23}=0$.

To summarize, we have shown that

$$
a_{13}=a_{23}=a_{24}=0 .
$$

Also, $a_{14}=0$. Therefore, the matrix $\Pi_{0}$ is block lower-diagonal. Its determinant is a nonzero multiple of $w^{2}$. The determinant of $\Pi_{0}$ is divisible by the determinant of its lower-diagonal $2 \times 2$ block

$$
\left(\begin{array}{ll}
a_{33} & a_{34} \\
a_{43} & a_{44}
\end{array}\right)=\left(\begin{array}{cc}
\alpha_{3}-\alpha_{1} f_{31} & \alpha_{2}-\alpha_{1} f_{32} \\
-v_{2}-\alpha_{1} f_{41} & v_{3}-\alpha_{1} f_{42}
\end{array}\right) .
$$

The only possibility is for this determinant to be a nonzero multiple of $w$, leading to the equation

$$
\left(\alpha_{3}-\alpha_{1} f_{31}\right)\left(v_{3}-\alpha_{1} f_{42}\right)+\left(\alpha_{2}-\alpha_{1} f_{32}\right)\left(v_{2}+\alpha_{1} f_{41}\right)=\lambda w
$$

for some $\lambda \in \mathbb{Q}$. Using $w=\alpha_{1} v_{1}+\alpha_{2} v_{2}+\alpha_{3} v_{3}$, we reduce the equation to $\alpha_{1}\left(-v_{1}-\alpha_{3} f_{42}-v_{3} f_{31}+\alpha_{1} f_{31} f_{42}+\alpha_{2} f_{41}-v_{2} f_{32}-\alpha_{1} f_{32} f_{41}\right)=(\lambda-1) w$. Since $w$ is not divisible by $\alpha_{1}$, we have $\lambda=1$, and the equation becomes

$$
-v_{1}-\alpha_{3} f_{42}-v_{3} f_{31}+\alpha_{1} f_{31} f_{42}+\alpha_{2} f_{41}-v_{2} f_{32}-\alpha_{1} f_{32} f_{41}=0 .
$$

Contradiction, since $v_{1}$ does not belong to the ideal generated by $\alpha_{1}, \alpha_{2}$, $\alpha_{3}, v_{2}, v_{3}$. Lemma 18 follows.

Entries $(4,3)$ and $(4,4)$ of equation $(28)$ now become

$$
\begin{aligned}
b_{44} f_{43} & =\alpha_{1} c_{41}+v_{3} c_{43}+v_{2}, \\
b_{44} & =\alpha_{1} c_{42}-\alpha_{2} c_{43}+\alpha_{3},
\end{aligned}
$$

implying

$$
v_{2}=f_{43}\left(\alpha_{1} c_{42}-\alpha_{2} c_{43}+\alpha_{3}\right)-\alpha_{1} c_{41}-v_{3} c_{43} .
$$

This is impossible, since $v_{2}$ does not lie in the ideal $\left(\alpha_{1}, \alpha_{2}, \alpha_{3}, v_{3}\right)$.

Therefore, $\theta$ is invertible and $\Upsilon$ is isomorphic to $M$ if $n>4$. Lemma 13 and Propositions 33, 32 follow in the case $n>4$.

If $n=4$, the sequences of degree shifts in $M^{0}, M^{1}$ (see formula (26)) are $(-3,-3,-5,-7)$ and $(-2,-4,-6,-6)$. These sequences are decreasing, and the matrices $\Theta_{0}, \Theta_{1}$ are block lower-triangular. By changing bases in $M^{0}$ and $M^{1}$ if necessary, we can assume that $\Theta_{0}$ and $\Theta_{1}$ are lower-triangular. The entry $b_{14}$ of $\Pi_{1}$ is a homogeneous linear polynomial in the $x$ 's. If $b_{14}=0$, 
Lemmas 14-18 hold for $n=4$ as well (with the simplification that $z_{0}=0$ in the proof of Lemma 14), and we are done. Assume now that $b_{14} \neq 0$. Then

- $f_{44}=0$ (from equation $(28)$, entry $(1,4)$ ),

- $c_{22}=0$ (equation $(28)$, entry $(2,4)$ ),

- $c_{33}=0$ (equation (28), entry $(3,4)$ ),

- $c_{32}=0$ (equation (28), entry $(3,4)$ ),

- $c_{44}=0$ (equation $(28)$, entry $(4,4)$ ),

- $f_{22}=0$ (equation $(27)$, entry $(2,4)$ ),

- $f_{11}=0$ (equation $(27)$, entry $(1,3)$ ),

- $f_{33}=0$ (equation $(27)$, entry $(3,4)$ ),

- $f_{32}=0$ (equation $(27)$, entry $(3,4)$ ).

Furthermore, $b_{14} f_{43}=\alpha_{1} c_{11}$ (equation (28), entry $(1,3)$ ), and $b_{14} f_{42}=\alpha_{2} c_{11}$ (equation (28), entry $(1,2)$ ). Since $\alpha_{1}$ and $\alpha_{2}$ are relatively prime, this is only possible if $c_{11}=0$, which, in turn, implies $f_{43}=0, f_{42}=0$. Next, $c_{21}=0$ (equation (28), entry $(2,2)$ ), and $c_{31}=0$ (equation $(28)$, entry $(3,2)$ ). From the remaining equations we derive

$$
\begin{array}{lll}
c_{41}=z v_{3}, & c_{42}=-z \alpha_{2}, & c_{43}=-z \alpha_{1}, \\
f_{21}=z a_{24}, & f_{31}=z a_{34}, & f_{41}=z a_{44},
\end{array}
$$

for some rational number $z$. Therefore, $\theta$ is null-homotopic, through the homotopy $(0, Z)$, where $Z$ is the $4 \times 4$ matrix with the only nonzero entry $z$ in the lower left corner. Contradiction. Lemma 13 and Propositions 33, 32 follow in the case $n=4$.

If $n=3$, the degree conditions force the coefficients

$$
a_{14}, b_{23}, c_{21}, c_{14}, c_{23}, c_{24}, c_{34}, f_{12}, f_{13}, f_{14}, f_{23}, f_{43}
$$

to be zero. By changing bases in $M^{0}, M^{1}$, if necessary, we can assume that $c_{13}=f_{24}=0$.

Lemma 19. $c_{44} \neq 0$ if $n=3$.

Proof. Suppose to the contrary that $c_{44}=0$. Three of the equations (27) reduce to

$$
\begin{aligned}
& 0=-\alpha_{1} f_{22}, \\
& 0=-\alpha_{1} f_{42}+v_{3} f_{44}, \\
& 0=-\alpha_{1} f_{32}+\alpha_{2} f_{33}+v_{3} f_{34},
\end{aligned}
$$

implying that

$$
f_{22}=f_{44}=f_{42}=f_{33}=0 .
$$

Equation $(28)$, entry $(2,4)$, implies $c_{22}=0$, while entry $(3,3)$ implies $c_{33}=$ $c_{31}=0$. From entry $(1,3)$ we derive $c_{11}=0$, and from the entry $(1,3)$ of (27) that $f_{11}=0$. The remaining entries of the two matrix equations force 
the other entries of $\Theta_{0}, \Theta_{1}$ to have the form

$$
\begin{array}{lll}
c_{12}=z_{0} b_{13}, & c_{32}=z_{0} b_{33}, & \\
c_{41}=-v_{3} z_{1}, & c_{42}=b_{43} z_{0}+\alpha_{2} z_{1}, & c_{43}=z_{1} \alpha_{1}, \\
f_{21}=-z_{1} a_{24}, & f_{31}=-z_{1} a_{34}-x v_{2}, & f_{41}=-z_{1} a_{44}, \\
f_{32}=z_{0} v_{3}, & f_{34}=z_{0} \alpha_{1} . &
\end{array}
$$

Therefore, we can write

$$
\Theta_{0}=Z_{1} \Lambda_{0}+\Pi_{1} Z_{0}, \quad \Theta_{1}=\Pi_{0} Z_{1}+Z_{0} \Lambda_{1},
$$

for matrices $Z_{0}, Z_{1}$ which have zero entries save for $z_{0}$ in $(3,2)$ in $Z_{0}$ and $-z_{1}$ in $(4,1)$ in $Z_{1}$, with $z_{0}, z_{1} \in \mathbb{Q}$. Thus, $\theta$ is homotopic to 0 and the lemma follows.

The proof of the lemma mimicked very closely the proof of Lemma 14 . The proofs of the following lemmas are omitted, since they are parallel to those of Lemmas 15-18.

Lemma 20. $f_{33} \neq 0$ if $n=3$.

LEMMA 21. $f_{11}=0$ if $n=3$ and $\theta$ is not an isomorphism.

LEMMA 22. $c_{22}=0$ if $n=3$ and $\theta$ is not an isomorphism.

LEMMA 23. $f_{44}=0$ if $n=3$ and $\theta$ is not an isomorphism.

We can assume that $f_{33}=c_{44}=1$. Entries $(4,3)$ and $(4,4)$ of equation (28) now become

$$
\begin{aligned}
b_{43} & =\alpha_{1} c_{41}+v_{3} c_{43}+v_{2}, \\
b_{43} f_{34} & =\alpha_{1} c_{42}-\alpha_{2} c_{43}+\alpha_{3},
\end{aligned}
$$

leading to a contradiction. Lemma 13 and Propositions 33, 32 follow in the remaining case $n=3$.

Shifts. Direct sum decompositions in the above propositions often contain terms shifted by $\langle 1\rangle$. In general, if there is a direct sum decomposition that contains $C\left(\Gamma_{1}\right)$ and $C\left(\Gamma_{2}\right)$ for a pair of graphs $\Gamma_{1}, \Gamma_{2}$, to determine whether $C\left(\Gamma_{2}\right)$ will be shifted by $\langle 1\rangle$ relative to $C\left(\Gamma_{1}\right)$ modify $\Gamma_{1}, \Gamma_{2}$ by substituting a pair of arcs for each wide edge of these graphs (right to left transformation in Figure 8). The graphs will turn into collections of arcs and circles inside a disc. The two collections share the set of boundary points. Glue them together along their boundaries to get a collection of circles on a 2 -sphere. If the number of circles plus half the number of boundary points is odd, there is a shift by $\langle 1\rangle$. Otherwise, $C\left(\Gamma_{1}\right)$ and $C\left(\Gamma_{2}\right)$ are unshifted relative to each other.

Closed graphs. We say that $\Gamma$ is closed if it has no boundary points. $C(\Gamma)$ is a 2-complex $\left(d^{2}=0\right)$ iff $\Gamma$ is closed. In this subsection we assume 
that $\Gamma$ is closed. Define the parity of $\Gamma$ (denoted $p(\Gamma)$ ) as the number of circles in the modification of $\Gamma$ described above (erase a neighborhood of each wide edge, and add two oriented arcs in place of this edge). Let $H(\Gamma)$ be the cohomology groups of $C(\Gamma)$. Since $C(\Gamma)$ has cohomology only in degree $p(\Gamma)$, we have

$$
H(\Gamma)=H^{p(\Gamma)}(C(\Gamma)) .
$$

Internal grading of $C(\Gamma)$ makes $H(\Gamma)$ into a $\mathbb{Z}$-graded $\mathbb{Q}$-vector space.

Proposition 34. The graded dimension of $H(\Gamma)$ is the invariant $P_{n}(\Gamma)$,

$$
\sum_{j \in \mathbb{Z}} \operatorname{dim} H^{j}(\Gamma) q^{j}=P_{n}(\Gamma)
$$

This follows from the direct sum decompositions obtained in this section and the well-known fact that skein relations in Figure 3 suffice to evaluate $P_{n}(\Gamma)$ for any graph $\Gamma$.

Examples of homologically regular pairs. We continue to assume that $\Gamma$ is closed, and hence $C(\Gamma)$ is a 2-complex. It is cohomology $H(\Gamma)$ concentrated in one degree only. $C(\Gamma)$ is the cyclic Koszul complex $\{\mathbf{a}, \mathbf{b}\}$ for a suitable pair $(\mathbf{a}, \mathbf{b})$ with $\mathbf{a b}=0$. If $H^{0}(\Gamma) \neq 0$, then $H^{1}(\Gamma)=0$, and the pair $(\mathbf{a}, \mathbf{b})$ is homologically regular, in the terminology of Section 2. If $H^{1}(\Gamma) \neq 0$, we can permute $a_{i}$ with $b_{i}$ in the sequences $\mathbf{a}, \mathbf{b}$, for some $i$, to produce a homologically regular pair. Thus, any closed graph gives rise to a homologically regular pair.

The simplest example of a homologically regular pair is $(\mathbf{0}, \mathbf{b})$, where $\mathbf{b}$ is a regular sequence.

For any pair, $H(\{\mathbf{a}, \mathbf{b}\})$ is a module over the ring $R$. Note that if $\mathbf{b}$ is regular, the cohomology $H^{0}(\{\mathbf{0}, \mathbf{b}\})$ is a cyclic module over $R$, isomorphic to $R /(\mathbf{b})$. Modifications of $(\mathbf{0}, \mathbf{b})$ using symmetries from the group $G$ in Section 2 do not change the cohomology and its module structure. All examples of homologically regular pairs given in Section 2 have the property that $H^{0}$ is a cyclic $R$-module.

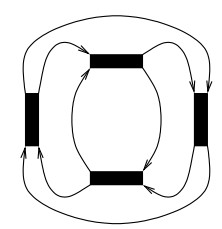

Fig. 43. Graph $\Gamma$

Curiously, homologically regular pairs $(\mathbf{a}, \mathbf{b})$ assigned to certain graphs $\Gamma$ have the property that $H^{0}$ is not a cyclic $R$-module. The simplest example of such $\Gamma$ is shown in Figure 43. We assume that $n>2$, and each oriented 
edge has one mark (not shown on the diagram). The graded dimension of $H^{0}(C(\Gamma)\langle 1\rangle)$ is

$$
[n][n-1](2[n-1]+[n-3])
$$

and has the form $q^{5-3 n}(2+x)$, where $x \in q \mathbb{Z}[q]$. Since the ring $R$ of polynomials in edge variables is nonnegatively graded, with $\mathbb{Q}$ in degree 0 , the cohomology of $C(\Gamma)\langle 1\rangle$ is not a cyclic module over $R$ (it is $\mathbb{Q} \oplus \mathbb{Q}$ in the lowest degree). Hence, the pair $(\mathbf{a}, \mathbf{b})$ assigned to this graph cannot be obtained from any pair of the form $\left(\mathbf{0}, \mathbf{b}^{\prime}\right)$ using symmetries from the group $G$ (see the end of Section 2). Thus, the pair assigned to $\Gamma$ is homologically regular and "intrinsically cyclic", unlike pairs $\left\{\mathbf{0}, \mathbf{b}^{\prime}\right\}$ whose cyclic Koszul complexes are just the Koszul complexes of $\mathbf{b}^{\prime}$ with collapsed grading.

\section{Tangle diagrams and complexes of factorizations}

Complexes of factorizations. To any additive category $\mathcal{C}$ associate the category $K(\mathcal{C})$ with objects being bounded complexes of objects of $\mathcal{C}$ and morphisms being morphisms of complexes up to homotopy. The category $K(\mathcal{C})$ is triangulated.

Recall that hmf $w$ is the category of graded factorizations with potential $w$ and finite-dimensional cohomology, up to homotopy. Let $K_{w}=K\left(\mathrm{hmf}_{w}\right)$, the homotopy category of $\operatorname{hmf}_{w}$. An object of $K_{w}$ is $\mathbb{Z} \oplus \mathbb{Z} \oplus \mathbb{Z}_{2}$-graded.

We distinguish the three shift functors in $K_{w}$, the shifts functors $\langle 1\rangle,\{1\}$ coming from $\mathrm{hmf}_{w}$ and the shift [1] in the category of complexes. These three functors pairwise commute.

EXAMPLE. If $w$ is a potential in the empty set of variables, $\mathrm{hmf}_{w}$ is the homotopy category of 2-periodic complexes of graded $\mathbb{Q}$-vector spaces with finite-dimensional cohomology. Any object of $\mathrm{hmf}_{w}$ is then isomorphic to its cohomology, which is a $\mathbb{Z} \oplus \mathbb{Z}_{2}$-graded vector space, and $\mathrm{hmf}_{w}$ is equivalent to the category of $\mathbb{Z} \oplus \mathbb{Z}_{2}$-graded finite-dimensional vector spaces. $K_{w}$ is equivalent to the homotopy category of this category, and therefore to the category of $\mathbb{Z} \oplus \mathbb{Z} \oplus \mathbb{Z}_{2}$-graded finite-dimensional vector spaces.

Tangles in a ball. By a tangle $L$ we mean a proper embedding of an oriented compact 1-manifold into a ball $B^{3}$. We fix a great circle on the boundary 2-sphere of $B^{3}$ and require that the boundary points of the embedded 1-manifold lie on this great circle. A diagram $D$ of $L$ is a generic projection of $L$ onto the plane of the great circle. An isotopy of a tangle should not move its boundary points.

A marked diagram (also denoted $D$ ) is a diagram with several marks placed on $D$ so that any segment bounded by crossings has at least one mark (see an example in Figure 44). Boundary points also count as marks. 


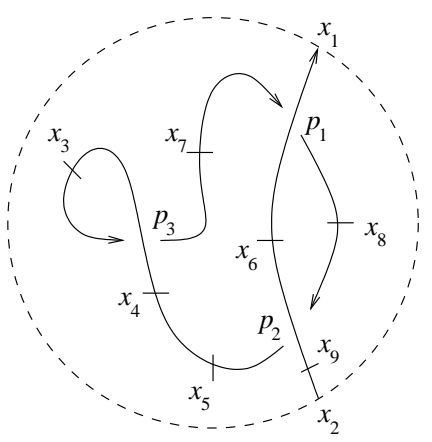

Fig. 44. A marked diagram of a tangle

Let $m(D)$ be the set of marks of $D$, and $\partial D$ the set of boundary points (it is a subset of $m(D)$ ). Let $R$ be the ring of polynomials in $x_{i}, i \in m(D)$, and $R^{\prime}$ the ring of polynomials in $x_{i}, i \in \partial D$.

We separate crossings of $D$ into positive and negative as explained in Figure 45.

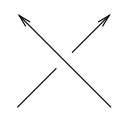

positive

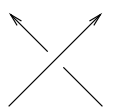

negative

Fig. 45. Positive and negative crossings

Given a crossing $p$, let $\Gamma^{0}, \Gamma^{1}$ be its two resolutions (see Figure 9). To a positive crossing assign the complex of factorizations (also see Figure 46)

$$
0 \rightarrow C\left(\Gamma^{0}\right)\{1-n\} \stackrel{\chi_{0}}{\longrightarrow} C\left(\Gamma^{1}\right)\{-n\} \rightarrow 0 .
$$

To a negative crossing assign the complex

$$
0 \rightarrow C\left(\Gamma^{1}\right)\{n\} \stackrel{\chi_{1}}{\longrightarrow} C\left(\Gamma^{0}\right)\{n-1\} \rightarrow 0 .
$$

In both cases we place $C\left(\Gamma^{0}\right)$ in cohomological degree 0 . Denote this complex by $C^{p}$.

$$
\begin{gathered}
X=[0 \rightarrow)_{0}\left(\{1-\mathrm{n}\} \rightarrow \prod_{1}[-\mathrm{n}\} \rightarrow 0\right] \\
Y=\left[0 \longrightarrow \prod_{-1}\{\mathrm{n}\} \rightarrow\right)_{0}(\{\mathrm{n}-1\} \rightarrow 0]
\end{gathered}
$$

Fig. 46. Complex assigned to a crossing

To $D$ associate the complex of factorizations $C(D)$ which is the tensor product of $C^{p}$ over all crossings $p$ of $L_{j}^{i}$ over all arcs $j \rightarrow i$, and of $A\langle 1\rangle$ over 
all crossingless markless circles of $D$ (if such exist). The tensoring is done over appropriate polynomial rings so that $C(D)$, as an $R$-module, is free of finite rank.

For instance, to produce $C(D)$ for $D$ in Figure 44, we tensor $C^{p_{1}}$ with $C^{p_{2}}$ over $\mathbb{Q}\left[x_{6}, x_{8}\right]$, and tensor the result with $C^{p_{3}}$ over $\mathbb{Q}\left[x_{7}\right]$.

We proceed by tensoring $C^{p_{1}} \otimes C^{p_{2}} \otimes C^{p_{3}}$ with $L_{5}^{4}$ over $\mathbb{Q}\left[x_{4}, x_{5}\right]$, and with $L_{2}^{9}$ over $\mathbb{Q}\left[x_{9}\right]$, so that

$$
C(D)=C^{p_{1}} \otimes C^{p_{2}} \otimes C^{p_{3}} \otimes L_{5}^{4} \otimes L_{2}^{9} .
$$

$C(D)$, for any diagram $D$, is a complex of graded $\left(R^{\prime}, w\right)$-factorizations, where

$$
w=\sum_{i \in \partial D} \pm x_{i}^{n+1},
$$

with signs determined by orientations of $D$ near boundary points. Thus, $C(D)$ is an object of the category $K_{w}$.

Proposition 35. The complexes $C(D)$ and $C\left(D^{\prime}\right)$ are canonically isomorphic if $D^{\prime}$ differs from $D$ only by marks.

Proof. This follows at once from Proposition 22.

Theorem 2. The complexes $C(D)$ and $C\left(D^{\prime}\right)$ are isomorphic in $K_{w}$ if $D$ and $D^{\prime}$ are two diagrams of the same tangle $L$.

Proof. It suffices to check the assertion when $D$ and $D^{\prime}$ are related by a single Reidemeister move. This is done in the next section.

Corollary 13. The isomorphism class of the object $C(D)$ in the category $K_{w}$ is an invariant of the tangle $L$.

Link homology. When $L$ is a link, the ring $R^{\prime}$ equals $\mathbb{Q}$, and $\operatorname{hmf}_{w}$ is isomorphic to the category of finite-dimensional $\mathbb{Z} \oplus \mathbb{Z} \oplus \mathbb{Z}_{2}$-graded $\mathbb{Q}$-vector spaces. Cohomology groups are nontrivial only in the cyclic degree which is the number of components of $L$ modulo 2. This reduces the grading of cohomology of $C(D)$ to $\mathbb{Z} \oplus \mathbb{Z}$.

Denote the resulting cohomology groups by

$$
H_{n}(D)=\bigoplus_{i, j \in \mathbb{Z}} H_{n}^{i, j}(D) .
$$

It is clear from the construction that the Euler characteristic of $H_{n}(D)$ is the polynomial $P_{n}(L)$,

$$
P_{n}(L)=\sum_{i, j \in \mathbb{Z}}(-1)^{i} q^{j} \operatorname{dim}_{\mathbb{Q}} H_{n}^{i, j}(D) .
$$

The isomorphism classes of the vector spaces $H_{n}^{i, j}(D)$ depend only on $L$. Denote by $h_{n}^{i, j}(L)$ the dimension of $H_{n}^{i, j}(D)$. This is an invariant of the 
link $L$. For each $n$, we can put them together into a 2 -variable polynomial invariant of $L$,

$$
h_{n}(L)=\sum_{i, j} t^{i} q^{j} h_{n}^{i, j}(L) .
$$

Reduced link homology. Choose a component of $L$, and a mark $i$ on $D$ that belongs to this component. For each resolution $\Gamma$ of $L$, the vector space $H(\Gamma)$ is a free module over the $\operatorname{ring} A \cong \mathbb{Q}\left[x_{i}\right] /\left(x_{i}^{n}\right)$. Let $\widetilde{\mathbb{Q}}$ be the one-dimensional graded $A$-module, placed in degree 0 , and

$$
\widetilde{C}(D):=C(D) \otimes_{A} \widetilde{\mathbb{Q}} \text {. }
$$

The complexes $\widetilde{C}(D)$ and $\widetilde{C}\left(D^{\prime}\right)$ are quasi-isomorphic if $D$ and $D^{\prime}$ represent the same link with the same preferred component. Denote the cohomology groups of $\widetilde{C}(D)$ by $\widetilde{H}_{n}^{i, j}(D)$, and their dimensions by $\widetilde{h}_{n}^{i, j}(L)$. Then

$$
\widetilde{h}_{n}(L):=\sum_{i, j} t^{i} q^{j} \widetilde{h}_{n}^{i, j}(L)
$$

is a two-variable polynomial invariant of $L$ with a preferred component. Its specialization to $t=-1$ is the one-variable polynomial $P_{n}(L) /[n]$, which is another common normalization for this one-variable specialization of HOMFLYPT.

\section{Invariance under Reidemeister moves}
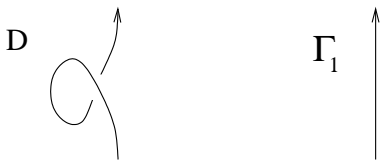

Fig. 47. Type I move

Type I move. Consider the Figure 47 case of the type I move. We follow the notations from the proof of Proposition 29. In particular, $\Gamma, \Gamma_{1}$, and $\Gamma_{2}$ are as in Figure 33. The complex $C(D)$ has the form

$$
0 \rightarrow C\left(\Gamma_{2}\right)\langle 1\rangle\{1-n\} \stackrel{\chi_{0}}{\longrightarrow} C(\Gamma)\{-n\} \rightarrow 0 .
$$

Let $\widetilde{\alpha}_{i}$, for $0 \leq i \leq n-1$, be the map

$$
\widetilde{\alpha}_{i}: C\left(\Gamma_{1}\right)\{2 i+2-2 n\} \rightarrow C\left(\Gamma_{2}\right)\langle 1\rangle\{1-n\}, \quad \widetilde{\alpha}_{i}=\sum_{j=0}^{i} m\left(x_{1}^{j} x_{2}^{i-j}\right) \iota^{\prime} .
$$

Let $Y_{1} \subset C\left(\Gamma_{2}\right)\langle 1\rangle\{1-n\}$ be the image of

$$
\bigoplus_{0 \leq i \leq n-2} C\left(\Gamma_{1}\right)\{2 i+2-2 n\}
$$


under the map

$$
\widetilde{\alpha}=\sum_{i=0}^{n-2} \widetilde{\alpha}_{i},
$$

and $Y_{2} \subset C\left(\Gamma_{2}\right)\langle 1\rangle\{1-n\}$ the image of $C\left(\Gamma_{1}\right)$ under the map $\widetilde{\alpha}_{n-1}$.

There is a direct sum decomposition in $\mathrm{hmf}_{w}$

$$
C\left(\Gamma_{2}\right)\langle 1\rangle\{1-n\} \cong Y_{1} \oplus Y_{2} .
$$

Furthermore, $\chi_{0}\left(Y_{2}\right)=0$, and the restriction of $\chi_{0}$ to $Y_{1}$ is an isomorphism from $Y_{1}$ to $C(\Gamma)\{-n\}$. Therefore, in the category $K_{w}$, the complex $C(D)$ is isomorphic to the direct sum of

$$
0 \rightarrow Y_{1} \stackrel{\cong}{\longrightarrow} C(\Gamma)\{-n\} \rightarrow 0 \text { and } 0 \rightarrow Y_{2} \rightarrow 0,
$$

with $Y_{2}$ in cohomological degree 0 . Since the first summand is contractible, $C(D)$ is isomorphic to $Y_{2} \cong C\left(\Gamma_{1}\right)$ in the category $K_{w}$.

The invariance under other cases of the type I move can be verified similarly.

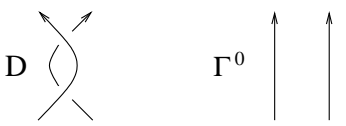

Fig. 48. Type IIa move

Type IIa move. Consider the diagrams $D$ and $\Gamma^{0}$ of Figure 48. The complex $C(D)$,

$$
0 \rightarrow C^{-1}(D) \stackrel{\partial^{-1}}{\longrightarrow} C^{0}(D) \stackrel{\partial^{0}}{\longrightarrow} C^{1}(D) \rightarrow 0,
$$

has the form (see Figure 49)

$$
0 \rightarrow C\left(\Gamma_{00}\right)\{1\} \stackrel{\left(f_{1}, f_{3}\right)^{t}}{\longrightarrow} \underset{C}{C} \stackrel{\oplus}{\oplus} \stackrel{\left(f_{2},-f_{4}\right)}{\longrightarrow} C\left(\Gamma_{11}\right)\{-1\} \rightarrow 0,
$$

where $f_{1}, f_{4}$ are given by the map $\chi_{1}$ (corresponding to the topology change in the lower halves of the diagrams $\left.\Gamma_{00}, \Gamma_{10}\right)$, and $f_{2}, f_{3}$ are given by $\chi_{0}$ (topology change in the upper halves of $\Gamma_{00}, \Gamma_{01}$ ).

We know that

$$
\begin{aligned}
& C\left(\Gamma_{10}\right) \cong C\left(\Gamma^{1}\right)\{1\} \oplus C\left(\Gamma^{1}\right)\{-1\}, \\
& C\left(\Gamma_{01}\right) \cong C\left(\Gamma^{0}\right), \\
& C\left(\Gamma_{00}\right) \cong C\left(\Gamma_{11}\right) \cong C\left(\Gamma^{1}\right) .
\end{aligned}
$$

where $\Gamma^{0}, \Gamma^{1}$ are the diagrams of Figure 8, and here and further on the isomorphisms are in the category $\mathrm{hmf}_{w}$, for the potential $w=x_{1}^{n+1}+x_{2}^{n+1}-$ $x_{3}^{n+1}-x_{4}^{n+1}$. 


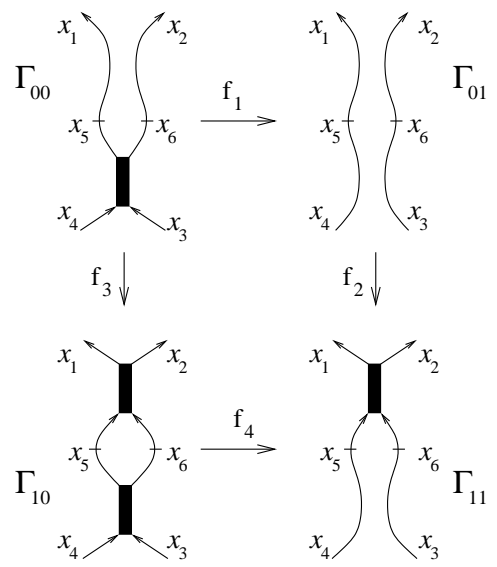

Fig. 49. Four resolutions of $D$ in the type IIa move

Fix isomorphisms (29), (30), (31). Under these isomorphisms, $f_{3}, f_{4}$ become two-component maps

$$
f_{3}=\left(f_{03}, f_{13}\right)^{t}, \quad f_{4}=\left(f_{04}, f_{14}\right),
$$

where for instance $f_{03}$ (respectively, $f_{13}$ ) is a degree 0 (respectively, degree 2 ) endomorphism of $C\left(\Gamma^{1}\right)$.

LEMMA 24.

(i) $C\left(\Gamma^{1}\right)$ has no negative degree endomorphisms in $\mathrm{hmf}_{w}$. The only degree 0 endomorphisms are multiples of the identity.

(ii) The space of degree 2 endomorphisms of $C\left(\Gamma^{1}\right)$ is 3-dimensional if $n>2$. Multiplications by $x_{1}, x_{2}, x_{3}, x_{4}$ span this space, with the only relation $m\left(x_{1}+x_{2}-x_{3}-x_{4}\right)=0$.

(iii) The space of degree 2 endomorphisms of $C\left(\Gamma^{1}\right)$ is 2-dimensional if $n=2$. Multiplications by $x_{1}, x_{2}, x_{3}, x_{4}$ span this space, with relations $m\left(x_{1}+x_{2}\right)=0$ and $m\left(x_{3}+x_{4}\right)=0$.

Proof. Since $C\left(\Gamma^{1}\right)\{1\}$ is the Koszul factorization for the pair

$$
\left(\left(u_{1}, u_{2}\right),\left(x_{1}+x_{2}-x_{3}-x_{4}, x_{1} x_{2}-x_{3} x_{4}\right)\right),
$$

the complex $\operatorname{Hom}_{R}\left(C\left(\Gamma^{1}\right), C\left(\Gamma^{1}\right)\right)$ is isomorphic to the Koszul complex of the sequence

$$
\left(x_{1}+x_{2}-x_{3}-x_{4}, x_{1} x_{2}-x_{3} x_{4}, u_{1}, u_{2}\right),
$$

with the grading collapsed from $\mathbb{Z}$ to $\mathbb{Z}_{2}$ (for the definition of $u_{1}, u_{2}$ see Section 6). This sequence is regular, so that the cohomology of this 2-complex is

$$
\mathbb{Q}\left[x_{1}, x_{2}, x_{3}, x_{4}\right] /\left(x_{1}+x_{2}-x_{3}-x_{4}, x_{1} x_{2}-x_{3} x_{4}, u_{1}, u_{2}\right) .
$$


Part (i) of the lemma follows, since this cohomology is isomorphic, as a graded $\mathbb{Q}$-algebra, to $\operatorname{End}_{\mathrm{HMF}}\left(C\left(\Gamma^{1}\right)\right)$. If $n>2$, all terms in (32) but the first are homogeneous polynomials in $x$ 's that are at least quadratic. Part (ii) follows. If $n=2$, the term $u_{2}$ is linear, and we get the additional relation $m\left(x_{3}+x_{4}\right)=0$.

REMARK. If $n=1$, there is nothing to prove, since $C(\Gamma)=0$ whenever $\Gamma$ has a wide edge, and the whole story becomes trivial.

From the lemma we deduce that $f_{03}$ and $f_{14}$ are rational multiples of the identity, while $f_{13}, f_{04}$ are multiplications by linear combinations of $x_{1}, x_{2}, x_{3}, x_{4}$.

$C(D)$ is a complex, so that $\partial^{0} \partial^{-1}=0$. We compute

$$
0=\partial^{0} \partial^{-1}=f_{2} f_{1}-f_{4} f_{3}=m\left(x_{1}-x_{3}\right)-f_{14} f_{13}-f_{04} f_{03} .
$$

Therefore, either $f_{14} \neq 0$, or $f_{03} \neq 0$, since multiplication by $x_{1}-x_{3}$ is a nontrivial endomorphism of $C\left(\Gamma^{1}\right)$.

Assume that $f_{14} \neq 0$. Then $f_{14}$ is a nonzero multiple of the identity. By rescaling, we normalize $f_{14}$ to be the identity.

Lemma 25. $f_{03} \neq 0$.

Proof. Suppose that $f_{03}=0$. Then $f_{13}=m\left(x_{1}-x_{3}\right)$. The composition of $f_{3}$ with the map $\chi_{1}$ in the opposite direction, where the topology change takes place around the upper wide edge of $\Gamma_{10}$, is multiplication by $x_{1}-x_{6}$, which is the same as multiplication by $x_{1}-x_{2}$ (since multiplications by $x_{2}$ and $x_{6}$ are homotopic endomorphisms of $C\left(\Gamma_{00}\right)$ ). The map $\chi_{1}$, restricted to the summand $C\left(\Gamma^{1}\right)\{-1\}$ of $C\left(\Gamma_{10}\right)$, is multiplication by some rational number $z$. Under the assumption $f_{03}=0$, we have

$$
m\left(x_{1}-x_{2}\right)=\chi_{1} f_{3}=\chi_{1} f_{13}=z \cdot m\left(x_{1}-x_{3}\right) .
$$

Therefore,

$$
m\left((1-z) x_{1}-x_{2}+z x_{3}\right)=0 .
$$

This is impossible, by Lemma 24. Lemma 25 follows.

Likewise, if $f_{03} \neq 0$, we can show that $f_{14} \neq 0$. Thus, both $f_{03}$ and $f_{14}$ are nonzero multiples of the identity morphism. We normalize so that each of them is the identity map. The differential

$$
\partial^{-1}: C^{-1}(D) \rightarrow C^{0}(D)
$$

is split injective. Since $f_{14}$ is the identity, the differential

$$
\partial^{0}: C^{0}(D) \rightarrow C^{1}(D)
$$

is split surjective. We can twist the direct sum decomposition

$$
C^{0}(D) \cong C\left(\Gamma^{0}\right) \oplus C\left(\Gamma^{1}\right)\{1\} \oplus C\left(\Gamma^{1}\right)\{-1\}
$$


so that $f_{03}, f_{14}$ become the only nonzero entries in the matrices describing the differentials $\partial^{-1}, \partial^{0}$, and $C(D)$ breaks into the direct sum of three complexes

$$
\begin{aligned}
0 & \rightarrow C\left(\Gamma^{0}\right) \quad \rightarrow 0, \\
0 \rightarrow C\left(\Gamma^{1}\right)\{1\} & \stackrel{\cong}{\rightarrow} C\left(\Gamma^{1}\right)\{1\} \\
0 & \rightarrow C\left(\Gamma^{1}\right)\{-1\} \stackrel{\cong}{\rightrightarrows} C\left(\Gamma^{1}\right)\{-1\} \rightarrow 0 .
\end{aligned}
$$

The last two complexes are contractible. Therefore, $C(D)$ and $C\left(\Gamma^{0}\right)$ are isomorphic in the category $K_{w}$.

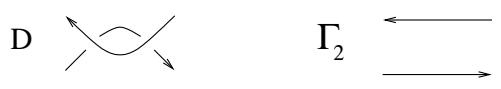

Fig. 50. Type IIb move

Type IIb move. Consider the diagrams $D$ and $\Gamma_{2}$ of Figure 50. The complex $C(D)$ has the form (see Figure 51)

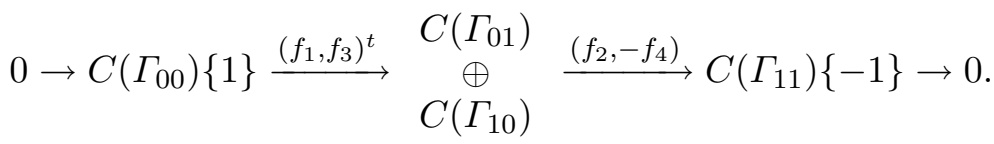

$$
\begin{aligned}
& \Gamma_{00} \\
& \mathrm{f}_{3} \quad \mathrm{f}_{2} \downarrow \\
& \left.\Gamma_{10}\right)\left(\stackrel{\mathrm{f}_{4}}{\longrightarrow} \prod \Gamma_{11}\right.
\end{aligned}
$$

Fig. 51. Commutative square of resolutions of $D$, type IIb move

There are isomorphisms in $\mathrm{hmf}_{w}$

$$
\begin{aligned}
C\left(\Gamma_{00}\right)\{1\} & \cong \bigoplus_{i=0}^{n-2} C\left(\Gamma_{1}\right)\langle 1\rangle\{3-n+2 i\}, \\
C\left(\Gamma_{11}\right)\{-1\} & \cong \bigoplus_{i=0}^{n-2} C\left(\Gamma_{1}\right)\langle 1\rangle\{1-n+2 i\}, \\
C\left(\Gamma_{10}\right) & \cong \bigoplus_{i=0}^{n-1} C\left(\Gamma_{1}\right)\langle 1\rangle\{1-n+2 i\},
\end{aligned}
$$




$$
C\left(\Gamma_{01}\right) \cong\left(\bigoplus_{i=0}^{n-3} C\left(\Gamma_{1}\right)\langle 1\rangle\{3-n+2 i\}\right) \oplus C\left(\Gamma_{2}\right),
$$

where $\Gamma_{1}$ and $\Gamma_{2}$ are as in Figure 36.

The proof of the invariance under type I move implies that $f_{4}$ is split surjective. Likewise, $f_{3}$ is split injective. Since the category $\mathrm{hmf}_{w}$ has splitting idempotents, we can decompose $C^{0}(D)$ into the direct sum

$$
C^{0}(D) \cong \operatorname{Im}\left(\partial^{-1}\right) \oplus Y_{1} \oplus Y_{2}
$$

so that $\partial^{0}$ restricts to an isomorphism from $Y_{1}$ to $C\left(\Gamma_{11}\right)\{-1\}$ and $\partial^{0} Y_{2}=0$. Therefore, $C(D)$ is isomorphic to the direct sum of complexes

$$
\begin{aligned}
0 & \rightarrow Y_{2} \quad \rightarrow 0, \\
0 \rightarrow C\left(\Gamma_{00}\right)\{1\} & \stackrel{\cong}{\rightarrow} \operatorname{Im}\left(\partial^{-1}\right) \\
0 & \rightarrow 0, \\
& Y_{1} \stackrel{\cong}{\rightrightarrows} C\left(\Gamma_{11}\right)\{-1\} \rightarrow 0 .
\end{aligned}
$$

From formulas (33)-(36) we obtain

$$
C^{0}(D) \cong C\left(\Gamma_{01}\right) \oplus C\left(\Gamma_{10}\right) \cong C\left(\Gamma_{00}\right)\{1\} \oplus C\left(\Gamma_{11}\right)\{-1\} \oplus C\left(\Gamma_{2}\right) .
$$

The category $\operatorname{hmf}_{w}$ is Krull-Schmidt (objects have unique direct sum decompositions into indecomposables). Therefore, $Y_{2} \cong C\left(\Gamma_{2}\right)$ and the complexes $C(D)$ and $0 \rightarrow C\left(\Gamma_{2}\right) \rightarrow 0$ are isomorphic. This concludes our proof of the invariance under the type IIb move.

Type III move. We need to show that $C(D)$ and $C\left(D^{\prime}\right)$ are isomorphic for $D, D^{\prime}$ of Figure 52 .

$\mathrm{D}$

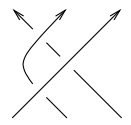

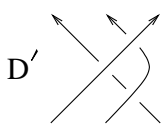

Fig. 52. Type III move

The diagram $D$ has eight resolutions, denoted $\Gamma_{i j k}$, for $i, j, k \in\{0,1\}$ (see Figure 53). The complex $C(D)\{-3 n\}$ has the form

$$
\begin{aligned}
& 0 \rightarrow C\left(\Gamma_{111}\right) \stackrel{d^{-3}}{\longrightarrow}\left(\begin{array}{l}
C\left(\Gamma_{011}\right)\{-1\} \\
C\left(\Gamma_{010}\right)\{-1\} \\
C\left(\Gamma_{110}\right)\{-1\}
\end{array}\right) \\
& \stackrel{d^{-2}}{\longrightarrow}\left(\begin{array}{c}
C\left(\Gamma_{100}\right)\{-2\} \\
C\left(\Gamma_{010}\right)\{-2\} \\
C\left(\Gamma_{001}\right)\{-2\}
\end{array}\right) \stackrel{d^{-1}}{\longrightarrow} C\left(\Gamma_{000}\right)\{-3\} \rightarrow 0
\end{aligned}
$$

with $C\left(\Gamma_{000}\right)\{-3\}$ in cohomological degree 0 . We depicted this complex in Figure 53. 


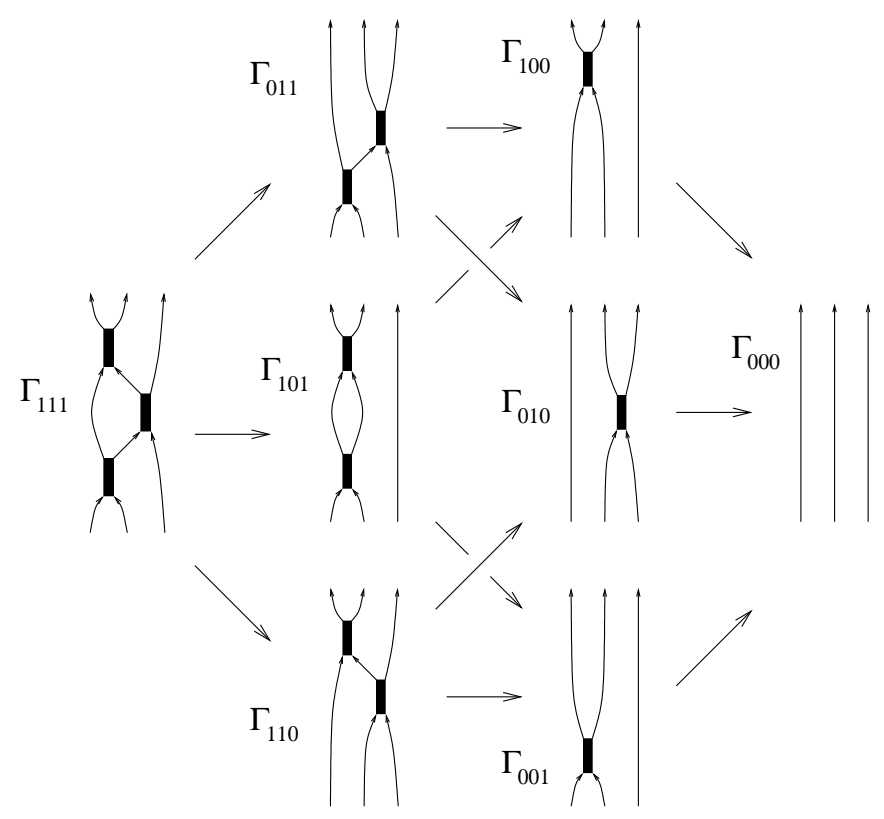

Fig. 53. Resolution cube of $D$

The diagrams $\Gamma_{100}$ and $\Gamma_{001}$ are isotopic, so that $C\left(\Gamma_{100}\right)$ and $C\left(\Gamma_{001}\right)$ are isomorphic factorizations. Moreover,

$$
C\left(\Gamma_{101}\right) \cong C\left(\Gamma_{100}\right)\{1\} \oplus C\left(\Gamma_{100}\right)\{-1\},
$$

and from Proposition 33 we know that

$$
C\left(\Gamma_{111}\right) \cong C\left(\Gamma_{100}\right) \oplus \Upsilon \text {. }
$$

The differential $d^{-3}$ is injective on $C\left(\Gamma_{100}\right) \subset C\left(\Gamma_{111}\right)$. In fact, its middle component (the map to $C\left(\Gamma_{101}\right)\{-1\}$ ) is injective, which follows from our construction of the inclusion $C\left(\Gamma_{100}\right) \subset C\left(\Gamma_{111}\right)$ and the proof of the invariance under the type IIa move.

The factorization $d^{-3}\left(C\left(\Gamma_{100}\right)\right)$ is a direct summand of $C^{-2}(D)\{-3 n\}$. Thus, $C(D)\{-3 n\}$ contains a contractible summand

$$
0 \rightarrow C\left(\Gamma_{100}\right) \stackrel{d^{-3}}{\longrightarrow} C\left(\Gamma_{100}\right) \rightarrow 0 .
$$

The direct sum decomposition (37) can be selected so that

$$
C\left(\Gamma_{101}\right)\{-1\} \cong p_{101} d^{-3} C\left(\Gamma_{100}\right) \oplus C\left(\Gamma_{100}\right)\{-2\}
$$

where $p_{101}$ is the projection onto the middle summand of $C^{-2}(D)\{-3 n\}$.

The differential $d^{-2}$ is injective on $C\left(\Gamma_{100}\right)\{-2\} \subset C\left(\Gamma_{101}\right)\{-1\}$, since its middle component is a nonzero multiple of the identity. Furthermore, the image of $C\left(\Gamma_{100}\right)\{-2\} \subset C\left(\Gamma_{101}\right)\{-1\}$ under $d^{-2}$ is a direct summand of $C^{-1}(D)$. Hence, the complex $C(D)\{-3 n\}$ contains a contractible direct 
summand isomorphic to

$$
0 \rightarrow C\left(\Gamma_{100}\right)\{-2\} \stackrel{d^{-2}}{\longrightarrow} C\left(\Gamma_{100}\right)\{-2\} \rightarrow 0 .
$$

After splitting off the contractible direct summands (38) and (39), the complex $C(D)\{-3 n\}$ reduces to the complex $C$ of the form

$$
\begin{aligned}
0 \rightarrow \Upsilon \stackrel{d^{-3}}{\longrightarrow}\left(\begin{array}{l}
C\left(\Gamma_{011}\right)\{-1\} \\
C\left(\Gamma_{110}\right)\{-1\}
\end{array}\right) \\
\stackrel{d^{-2}}{\longrightarrow}\left(\begin{array}{l}
C\left(\Gamma_{010}\right)\{-2\} \\
C\left(\Gamma_{100}\right)\{-2\}
\end{array}\right) \stackrel{d^{-1}}{\longrightarrow} C\left(\Gamma_{000}\right)\{-3\} \rightarrow 0
\end{aligned}
$$

(see Figure 54). To the diagram $Y$ on the left of the figure we assign the factorization $\Upsilon$.

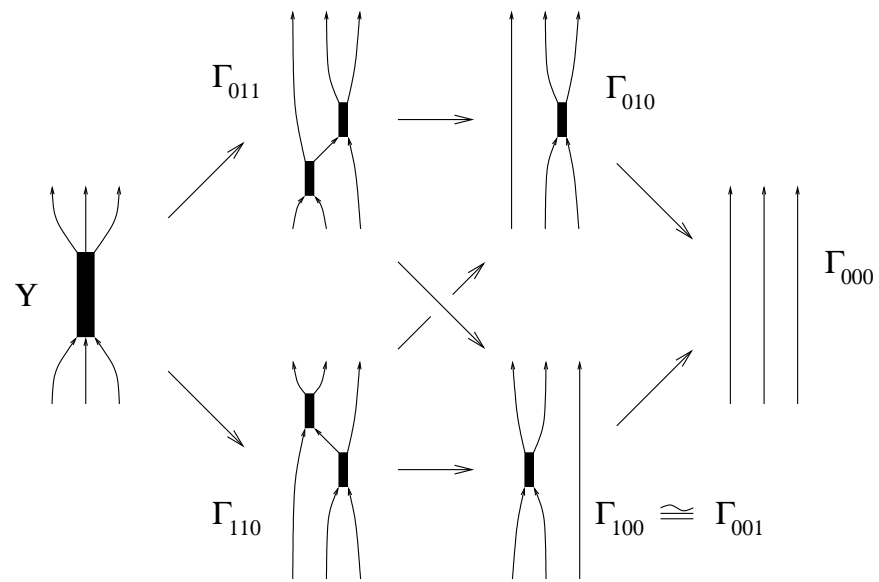

Fig. 54. Complex $C$

In the rest of the section we assume that $n>2$ (proofs for $n=2$ are easier since then $\Upsilon=0$ ).

Lemma 26. For each arrow in Figure 54 with some diagram $Z_{1}$ as the source and $Z_{2}$ as the target, the space of grading-preserving morphisms

$$
\mathrm{Hom}_{\mathrm{hmf}}\left(C\left(Z_{1}\right), C\left(Z_{2}\right)\{-1\}\right)
$$

is one-dimensional.

Proof. Straightforward, similar to the proof of Corollary 12. The details are omitted.

Denote by $c_{1}, \ldots, c_{8}$ the nontrivial morphisms from the above lemma. Each morphism is determined up to multiplication by a nonzero rational number. Figure 55 shows how the indices $i$ of $c_{i}$ 's match the eight arrows of Figure 54 . We choose $c_{i}$ 's so that each square in Figure 55 commutes. 


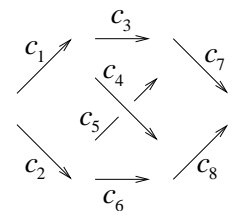

Fig. 55. Indices and arrows

The differential in $C$ is grading-preserving. Therefore, it has the form

$$
d^{-3}=\left(\begin{array}{c}
\lambda_{1} c_{1} \\
\lambda_{2} c_{2}
\end{array}\right), \quad d^{-2}=\left(\begin{array}{cc}
\lambda_{3} c_{3} & \lambda_{5} c_{5} \\
\lambda_{4} c_{4} & \lambda_{6} c_{6}
\end{array}\right), \quad d^{-1}=\left(\begin{array}{c}
\lambda_{7} c_{7} \\
\lambda_{8} c_{8}
\end{array}\right)
$$

for some rational numbers $\lambda_{1}, \ldots, \lambda_{8}$.

Lemma 27. For any two composable arrows $Z_{1} \rightarrow Z_{2} \rightarrow Z_{3}$ in Figure 54, the corresponding homomorphism of factorizations

$$
c_{j} c_{i}: C\left(Z_{1}\right) \rightarrow C\left(Z_{3}\right)\{-2\}
$$

is nonzero in $\mathrm{hmf}_{w}$.

Proof. For instance, to show that $c_{7} c_{3} \neq 0$, we note that this composition is the product of two $\chi_{1}$ maps (up to rescaling by a nonzero rational number), one for each wide edge of $\Gamma_{011}$. Compose it with the "dual" product of two $\chi_{0}$ maps, as in Figure 56. Assign variables $x_{1}, \ldots, x_{6}$ to the endpoints of our diagrams in the same way as in Figure 42. Then the Figure 56 map is $m\left(x_{1}-x_{5}\right) m\left(x_{2}-x_{6}\right)$. This is a nontrivial endomorphism of $C\left(\Gamma_{000}\right)$, so that the composition $c_{7} c_{3}$ is nonzero.

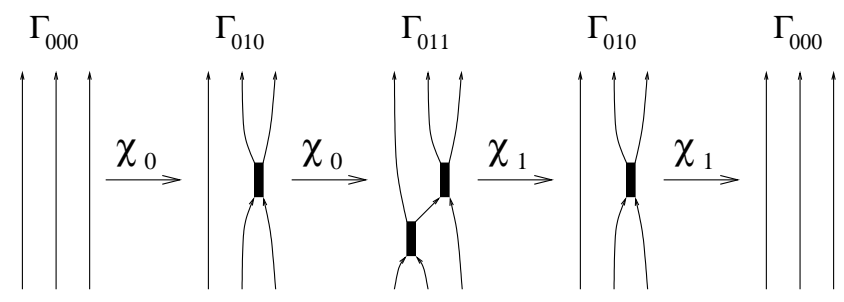

Fig. 56. Composing $c_{7} c_{3}$ with its dual

Identical arguments take care of the other three compositions that end in $\Gamma_{000}$. Similar but longer computations can be used to check nontriviality of each of the four compositions $c_{j} c_{i}$ that start at $Y$.

The category $K_{w}$ has an automorphism (tensoring with the complex assigned to the inverse braid of $D$ ) that takes $C(D)$ to $C\left(D^{\prime \prime}\right)$ where $D^{\prime \prime}$ is the diagram made up of three disjoint oriented arcs. Since $C\left(D^{\prime \prime}\right)$ is indecomposable in $K_{w}$, the same is true of $C(D)$ and $C \cong C(D)$. Indecomposability of $C$ together with the above lemma implies that none of the coefficients 
$\lambda_{1}, \ldots, \lambda_{8}$ is 0 . By rescaling, we can set

$$
\lambda_{1}=\lambda_{2}=\lambda_{4}=\lambda_{5}=\lambda_{7}=\lambda_{8}=1, \quad \lambda_{3}=\lambda_{6}=-1 .
$$

The complex $C$ is therefore invariant under the "flip" which transposes $x_{1}$ with $x_{3}$ and $x_{4}$ with $x_{6}$. This flip takes $C(D)$ to $C\left(D^{\prime}\right)$, thus, $C$ is isomorphic in $K_{w}$ to $C\left(D^{\prime}\right)$. We have $C(D) \cong C \cong C\left(D^{\prime}\right)$. The invariance under the Reidemeister move III of Figure 52 follows.

9. Factorizations and 2-dimensional TQFTs with corners. Let $I$ be a finite set and $s: I \rightarrow\{1,-1\}$ a map, which we call the orientation map. We say that $s$ is balanced if the sets $s^{-1}(1)$ and $s^{-1}(-1)$ have the same cardinality (i.e., $s$ takes as many elements of $I$ to 1 as it does to -1$)$. From now on we assume that $s$ is balanced.

To $(I, s)$ we assign the category $\operatorname{Cob}_{I, s}$ whose objects are oriented onedimensional manifolds $N$ with boundary $I$ such that the orientation of $N$ induces the orientation $s$ on $I=\partial N$. Morphisms from $N_{0}$ to $N_{1}$ are oriented 2-dimensional surfaces $S$ with boundary $N_{0} \cup-N_{1} \cup I \times[0,1]$ and corners $I \times\{0\} \sqcup I \times\{1\}$.

Let $R_{I}$ be the ring of polynomials in $x_{i}, i \in I$, and set

$$
w(I, s)=\sum_{i \in I} s(i) x_{i}^{n+1} \in R_{I} .
$$

To an object $N \in \mathrm{Cob}_{I, s}$ we can assign a factorization $C(N)$ with potential $w(I, s)$. The factorization is the tensor product (over $\mathbb{Q}$ ) of $L_{j}^{i}$ over all arcs $j \rightarrow i$ in $N$, and of $A\langle 1\rangle$, one for each circle in $N$. We would like to extend this assignment to a functor from $\mathrm{Cob}_{I, s}$ to the category of factorizations. To do this, pick a morphism $S$ in $\mathrm{Cob}_{I, s}$ and write it as a composition of simple cobordisms (cobordisms with only one critical point). To the cobordisms of creation and annihilation of a circle we assign the maps $\iota, \varepsilon$, defined in Section 4. To the saddle point cobordism between 1-manifolds $N_{0}, N_{1}$ of Figure 57 we want to associate a map

$$
\eta: C\left(N_{0}\right) \rightarrow C\left(N_{1}\right)\langle 1\rangle\{1-n\} .
$$

To define $\eta$, assign labels $a_{1}, a_{2}, b_{1}, b_{2}$ to the components of $N_{0}, N_{1}$ as indicated in Figure 57.
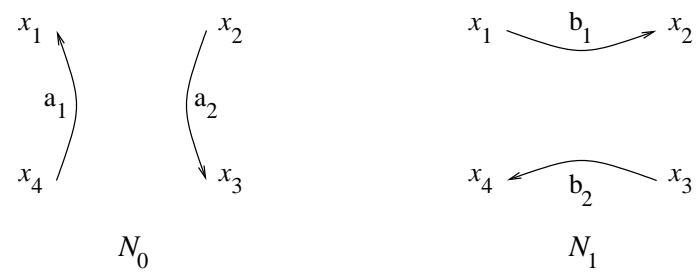

Fig. 57 
The factorization $C\left(N_{0}\right)$ has the form

$$
\left(\begin{array}{c}
R(\emptyset) \\
R\left(a_{1} a_{2}\right)\{2-2 n\}
\end{array}\right) \stackrel{P_{0}}{\longrightarrow}\left(\begin{array}{c}
R\left(a_{1}\right)\{1-n\} \\
R\left(a_{2}\right)\{1-n\}
\end{array}\right) \stackrel{P_{0}^{\prime}}{\longrightarrow}\left(\begin{array}{c}
R(\emptyset) \\
R\left(a_{1} a_{2}\right)\{2-2 n\}
\end{array}\right),
$$

where

$$
P_{0}=\left(\begin{array}{cc}
\pi_{14} & x_{3}-x_{2} \\
\pi_{32} & x_{4}-x_{1}
\end{array}\right), \quad P_{0}^{\prime}=\left(\begin{array}{cc}
x_{1}-x_{4} & x_{3}-x_{2} \\
\pi_{32} & -\pi_{14}
\end{array}\right),
$$

and $R=\mathbb{Q}\left[x_{1}, x_{2}, x_{3}, x_{4}\right]$.

The factorization $C\left(N_{1}\right)\langle 1\rangle$ is given by

$$
\left(\begin{array}{l}
R\left(b_{1}\right)\{1-n\} \\
R\left(b_{2}\right)\{1-n\}
\end{array}\right) \stackrel{P_{1}}{\longrightarrow}\left(\begin{array}{c}
R(\emptyset) \\
R\left(b_{1} b_{2}\right)\{2-2 n\}
\end{array}\right) \stackrel{P_{1}^{\prime}}{\longrightarrow}\left(\begin{array}{c}
R\left(b_{1}\right)\{1-n\} \\
R\left(b_{2}\right)\{1-n\}
\end{array}\right),
$$

where

$$
P_{1}=\left(\begin{array}{cc}
x_{2}-x_{1} & x_{4}-x_{3} \\
-\pi_{34} & \pi_{12}
\end{array}\right), \quad P_{1}^{\prime}=\left(\begin{array}{cc}
-t_{12} & x_{4}-x_{3} \\
-\pi_{34} & x_{1}-x_{2}
\end{array}\right),
$$

and $R=\mathbb{Q}\left[x_{1}, x_{2}, x_{3}, x_{4}\right]$.

$\eta$ is given by the pair of matrices

$$
\begin{aligned}
& \left(\begin{array}{cc}
e_{123}+e_{124}+\left(x_{4}-x_{3}\right) r & 1 \\
-e_{134}-e_{234}+\left(x_{1}-x_{2}\right) r & 1
\end{array}\right), \\
& \left(\begin{array}{cc}
-1 & 1 \\
-e_{123}-e_{234}+\left(x_{1}-x_{4}\right) r & -e_{134}-e_{124}+\left(x_{3}-x_{2}\right) r
\end{array}\right),
\end{aligned}
$$

with

$$
e_{i j k}=\sum_{a+b+c=n-1} x_{i}^{a} x_{j}^{b} x_{k}^{c}
$$

and $r$ being an arbitrary polynomial of degree $n-2$ in $x_{1}, \ldots, x_{4}$. Up to chain homotopy, $\eta$ does not depend on $r$.

$\eta$ generates $\operatorname{Hom}_{\mathrm{HMF}}\left(C\left(N_{0}\right), C\left(N_{1}\right)\langle 1\rangle\right)$ as an $R$-module, and we should assign $\eta$ to the saddle point cobordism. There is a problem, though. The diagrams in Figure 57 are invariant under rotation by $180^{\circ}$, but $\eta$ acquires a sign after the rotation. Namely, if we transpose $x_{1}$ with $x_{3}, x_{2}$ with $x_{4}$, $a_{1}$ with $a_{2}$, and $b_{1}$ with $b_{2}$ in the formula for $\eta$, the resulting map is $-\eta$. Thus, $\eta$ can be canonically defined only up to sign.

If the saddle point cobordism takes place not between arcs, but between components of $N_{0}, N_{1}$, some of which are circles, we add marks to each component, select a suitable pair of arcs bounded by marks (and, possibly, by boundary points), apply $\eta$ to that pair, and finally erase marks. It is easy to see that, up to sign, the resulting map does not depend on the intermediate choices. 
Let $\mathrm{H}_{I, s}^{\prime}$ be the category with the same objects as $\mathrm{hmf}_{w(I, s)}$ but morphisms being the Ext groups $\operatorname{Ext}_{\mathrm{HMF}}\left(M_{0}, M_{1}\right)$, with $f$ and $-f$ identified for all $f \in \operatorname{Ext}_{\mathrm{HMF}}\left(M_{0}, M_{1}\right)$. We use Ext groups rather than just Hom's since the shift $\langle 1\rangle$ is built into $\iota, \varepsilon$, and $\eta$.

Given a surface $S$ which is an object of $\mathrm{Cob}_{I, S}$, write it as a product of cobordisms with only one critical point and define $C(S)$ as the corresponding product of $\iota, \varepsilon$, and $\eta$ 's. Recall that our definition of $\varepsilon$ contained a parameter $\zeta \in \mathbb{Q}^{*}$. To make $\varepsilon$ compatible with $\eta$ we must set $\zeta$ to either $1 /(n+1)$ or $-1 /(n+1)$ (so that $\varepsilon \eta= \pm$ Id if the saddle point cobordism goes from an arc to the union of a circle and an arc).

Proposition 36. $\pm C(S)$ does not depend on the presentation of $S$ as a product of elementary cobordisms.

The proof is left to the reader.

Thus, we obtain a functor from the cobordism category $\operatorname{Cob}_{I, s}$ to the category $\mathrm{H}_{I, s}^{\prime}$. The shifts $v_{1}(S), v_{2}(S)$ in

$$
\pm C(S): C\left(N_{0}\right) \rightarrow C\left(N_{1}\right)\left\langle v_{1}(S)\right\rangle\left\{v_{2}(S)\right\}
$$

are as follows. Glue $N_{0}$ and $N_{1}$ along the common boundary $I$, and count the number $v$ of components in the closed 1-manifold that results. $v_{1}(S)$ is the parity of $v+|I| / 2$, while

$$
v_{2}(S)=(n-1)\left(\chi(S)-\frac{|I|}{2}\right)
$$

where $\chi(S)$ is the Euler characteristic of $S$.

2-functor. In the above construction we fixed the boundary of onemanifolds. If we put together all categories $\mathrm{Cob}_{I, s}$ over various $I$ and $s$ (and consider decompositions of $I$ into pairs of disjoint sets, to view $N$ with $\partial N=I$ as a morphism) we get a 2-category of oriented surfaces with corners. The functors

$$
C: \mathrm{Cob}_{I, s} \rightarrow \mathrm{H}_{I, s}^{\prime}
$$

extend to a 2-functor from this 2-category of cobordisms to the 2-category of factorizations with potentials as objects, factorizations as morphisms, and elements of Ext groups between factorizations (with $f$ and $-f$ identified) as 2-morphisms. We leave the details to the reader.

10. Projective invariance for cobordisms of tangles. As before, we consider oriented tangles in a ball $B^{3}$. Fix a great circle on the ball's boundary, choose a finite subset $I$ of this circle and a balanced "orientation" function $s: I \rightarrow\{1,-1\}$. Let $\mathrm{TC}_{I, s}$ be the category of tangle cobordisms with objects oriented tangles $L$ in $B^{3}$ with oriented boundary $(I, s)$ and morphisms from $L_{0}$ to $L_{1}$ oriented surfaces $S$ embedded in $B^{3} \times[0,1]$ with boundary 


$$
\partial S=L_{0} \times\{0\} \cup L_{1} \times\{1\} \cup I \times[0,1]
$$

and corners $I \times\{0\} \cup I \times\{1\}$, up to isotopy that fixes the boundary.

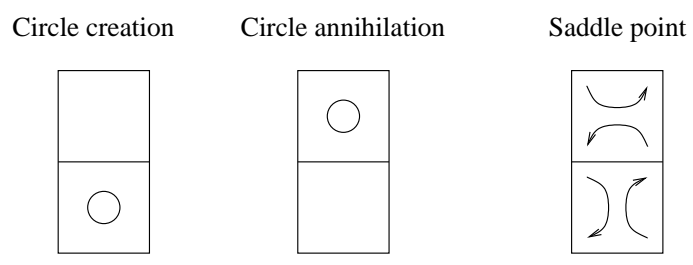

Fig. 58. Movie moves of simple cobordisms

A cobordism $S$ admits a combinatorial description via a sequence of plane diagrams of its cross-sections with $B^{3} \times k$, for various $k \in[0,1]$. Each consecutive pair of diagrams differ either by a Reidemeister move, or a Morse move, the latter describing a simple cobordism with one critical point (Figure 58). Such sequences are referred to as movies. Two sequences describe the same cobordism if they can be connected through a finite sequence of movie moves, shown in Figures 59,60. We assume that the reader is familiar with this theory, and refer to [CS1], [CS2] and references therein for details.
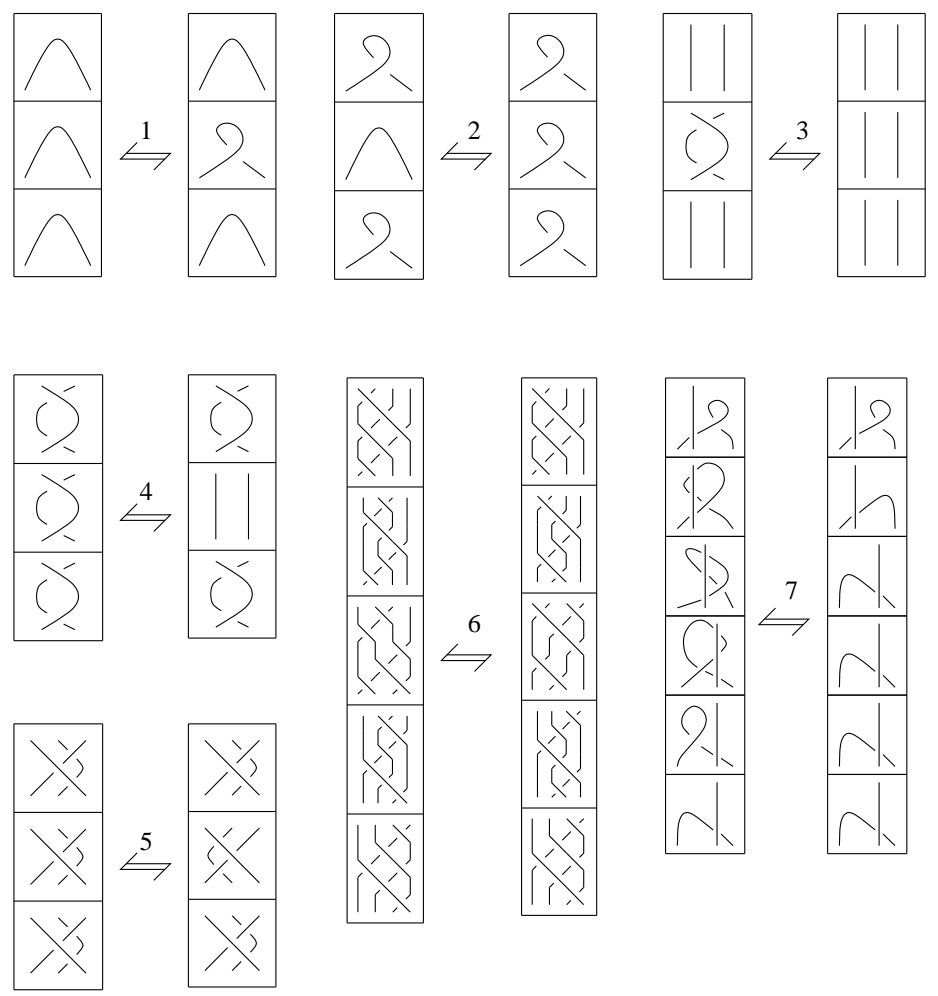

Fig. 59. Movie moves 1-7 

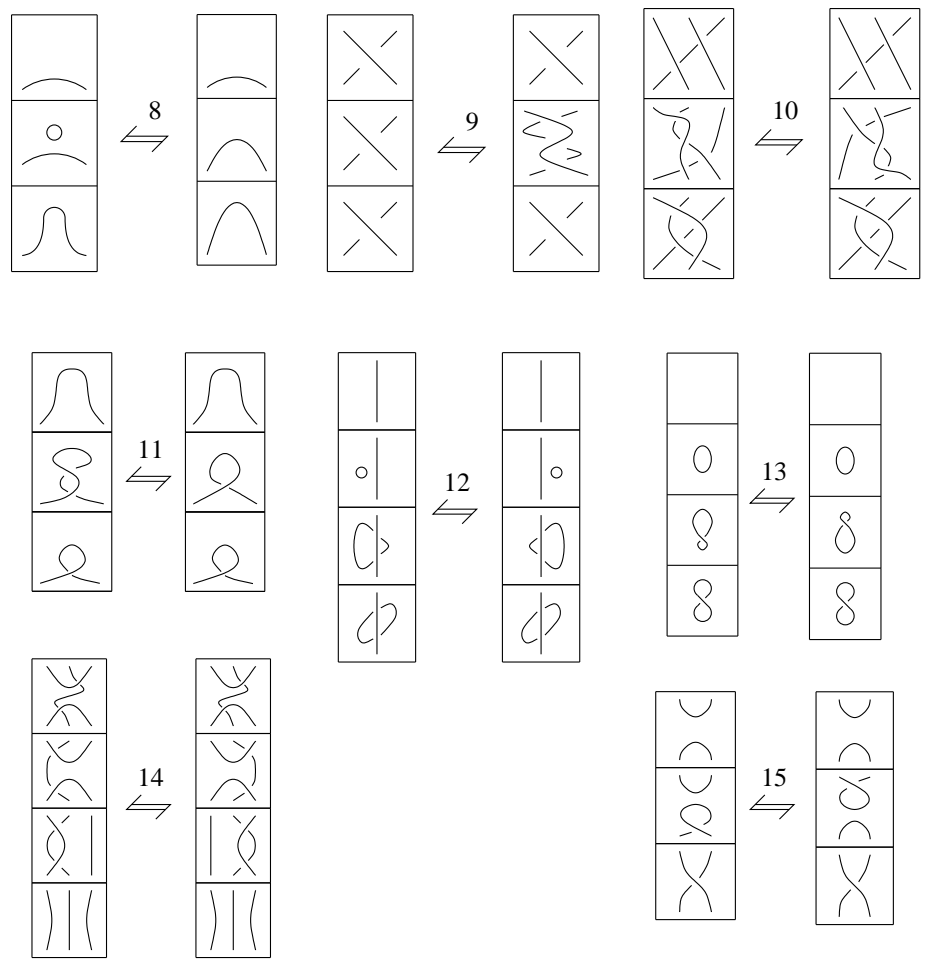

Fig. 60. Movie moves $8-15$

Let $w=w(I, s)$ be the potential defined in the preceding section. Earlier, we associated an isomorphism $C\left(D_{1}\right) \cong C\left(D_{2}\right)$ in the category $K_{w}$ to a Reidemeister move between the tangle diagrams $D_{1}$ and $D_{2}$. Aping the last section, assign the maps $\iota, \varepsilon, \eta$ to the circle creation, circle annihilation, and saddle point moves (see Figure 58), respectively.

Given a movie $z=\left(z_{0}, \ldots, z_{m}\right)$ representing a surface $S$, assign to $z$ the morphism in $K_{w}$ :

$$
C(z): C\left(z_{0}\right) \rightarrow C\left(z_{m}\right)\left\langle v_{1}(S)\right\rangle\left\{v_{2}(S)\right\}
$$

by composing the maps associated to each move $z_{i} \rightarrow z_{i+1}$ in $z$. The quantities $v_{1}(S), v_{2}(S)$ were defined at the end of the previous section.

Proposition 37. Up to overall multiplication by nonzero rational numbers, the map $C(z)$ (viewed as a morphism in the category $K_{w}$ ) does not depend on the movie presentation $z$ of $S$.

Proof. For a given movie move of Figures 59, 60, denote the top frame by $b_{1}$, the bottom frame by $b_{2}$, the left movie by $S_{l}$ and the right movie by $S_{r}$. We need to show that the morphisms $C\left(S_{l}\right)$ and $C\left(S_{r}\right)$ are proportional, $C\left(S_{l}\right)=\lambda C\left(S_{r}\right)$ for some $\lambda \in \mathbb{Q}^{*}$. 
The movies $S_{l}$ and $S_{r}$ in movie move 6 are compositions of Reidemeister moves. Therefore,

$$
C\left(S_{l}\right), C\left(S_{r}\right): C\left(b_{1}\right) \rightarrow C\left(b_{2}\right)
$$

are isomorphisms. Let $b$ be the tangle diagram which consists of four parallel disjoint segments (the crossingless diagram of the trivial braid). There is an automorphism of $K_{w}$ (tensoring with $C\left(b_{1}^{\prime}\right)$, where $b_{1}^{\prime}$ is the "inverse" of the braid diagram $b_{1}$ ) which takes $C\left(b_{1}\right)$ to $C(b)$. Therefore,

$$
\operatorname{Hom}_{K_{w}}\left(C\left(b_{1}\right), C\left(b_{1}\right)\right) \cong \operatorname{Hom}_{K_{w}}(C(b), C(b)) .
$$

The vector space on the right hand side is isomorphic to $\mathbb{Q}$ (the only degree 0 endomorphisms of $C(b)$ are multiples of the identity). Hence

$$
\operatorname{Hom}_{K_{w}}\left(C\left(b_{1}\right), C\left(b_{2}\right)\right) \cong \operatorname{Hom}_{K_{w}}\left(C\left(b_{1}\right), C\left(b_{1}\right)\right) \cong \mathbb{Q}
$$

(the isomorphisms are not canonical, though), and $C\left(S_{l}\right), C\left(S_{r}\right)$ are proportional.

This argument applies to moves 1, 2, 3, 4, 5, 7, 9, 10, 11 as well, and to all versions of these moves (various orientations, overcrossing/undercrossing variations, etc.)

Move 8 follows from the compatibility of $\iota, \varepsilon$, and $\eta$ (see Section 9 ).

In move 12 , the maps

$$
C\left(S_{l}\right), C\left(S_{r}\right): C\left(b_{1}\right) \rightarrow C\left(b_{2}\right)\langle 1\rangle\{n-1\}
$$

are nontrivial and lie in the one-dimensional $\mathbb{Q}$-vector space

$$
\operatorname{Hom}_{K_{w}}\left(C\left(b_{1}\right), C\left(b_{2}\right)\langle 1\rangle\{n-1\}\right) .
$$

Therefore, the maps are proportional. The same argument works for other versions of this move, and for all versions of moves 13,14,15 (for moves $14,15$ change $\{n-1\}$ to $\{1-n\})$. Proposition 37 follows.

The above proof is based on the observation that the space of homs between $C\left(b_{1}\right)$ and $C\left(b_{2}\right)$ (with a suitable shift) is one-dimensional. The same approach was used in [Kh4] to show functoriality of the homology theory $\mathcal{H}$ from [Kh1] (see Jacobsson [J] for a different proof), and by Dror Bar-Natan [BN2] to prove functoriality of his refinement of $\mathcal{H}$.

We denote by $C(S)$ the set $\left\{\lambda C(z) \mid \lambda \in \mathbb{Q}^{*}\right\}$. This is an invariant of the cobordism $S$.

In particular, given a diagram $D$ of a tangle $L$, the object $C(D)$ of $K_{w}$ is canonically (up to rescaling) associated to $L$. We denote this object by $C(L)$ (recall that it is a complex of graded matrix factorizations, treated as an object of $K_{w}$ ).

Given a diagram $D$ of an oriented link $L$, the homology groups $H_{n}(D)$ are $\mathbb{Q}$-vector spaces canonically assigned to $L$ (up to overall rescaling by 
nonzero rational numbers). We denote these groups by $H_{n}(L)$ and their graded summands by $H_{n}^{i, j}(L)$.

Thus, an oriented link cobordism $S$ between $L_{0}$ and $L_{1}$ induces a homomorphism

$$
C(S): H_{n}\left(L_{0}\right) \rightarrow H_{n}\left(L_{1}\right),
$$

well-defined up to rescalings by nonzero rationals, and for each $i, j$ restricts to homomorphisms

$$
H_{n}^{i, j}\left(L_{0}\right) \rightarrow H_{n}^{i, j+(1-n) \chi(S)}\left(L_{1}\right),
$$

where $\chi(S)$ is the Euler characteristic of $S$. The collection of maps $C(S)$ over all link cobordisms $S$ is a functor from the category of link cobordisms to the category of bigraded $\mathbb{Q}$-vector spaces (with morphisms being graded linear maps with the equivalence relation $f \sim \lambda f$ for $\lambda \in \mathbb{Q}^{*}$ ). The Euler characteristic of $H_{n}(L)$ is the polynomial

$$
P_{n}(L)=\sum_{i, j}(-1)^{i} q^{j} \operatorname{dim}_{\mathbb{Q}} H_{n}^{i, j}(L) .
$$

2-functor. So far we considered tangles with a fixed oriented boundary $(I, s)$. By switching from tangles in a ball to tangles in $\mathbb{R}^{2} \times[0,1]$ and varying possible boundaries one can form the 2-category of tangle cobordisms (see $[\mathrm{F}],[\mathrm{BL}]$, and references therein). Our construction can be extended to a 2 -functor from the 2-category of oriented tangle cobordisms to a 2-category with potentials $w(I, s)$ as objects, complexes of matrix factorizations as 1morphisms, and homomorphisms between (suitably shifted) complexes as 2-morphisms (of course, we will have to quotient by null-homotopic morphisms, and identify morphisms that are multiples of each other, $f \cong \lambda f$ for $\left.\lambda \in \mathbb{Q}^{*}\right)$. This 2-functor is braided monoidal.

11. A generalization. Each complex simple Lie algebra $\mathfrak{g}$ gives rise to a polynomial invariant of links whose components are decorated by finitedimensional irreducible representations of $\mathfrak{g}$ (see $[\mathrm{RT}]$ ). The polynomial $P_{n}$ results if $\mathfrak{g}=\mathfrak{s l}_{n}$ and every component is assigned the fundamental $n$-dimensional representation $V$ of $\mathfrak{s l}_{n}$. Murakami, Ohtsuki, and Yamada [MOY] develop a calculus of trivalent graphs that helps understand the polynomial invariant of links with components colored by arbitrary exterior powers of $V$ (the $i$ th exterior power $\Lambda^{i} V$ is often called the $i$ th fundamental representation of $\left.\mathfrak{s l}_{n}\right)$. Although their construction also extends the invariant to spacial trivalent graphs, here we will only look at planar graphs. Each edge of a graph is oriented and labeled by a number from 1 to $n-1$. Every vertex is trivalent and the sum of the labels at the edges entering the vertex minus the sum of the labels at the edges leaving it is a multiple of $n$. 


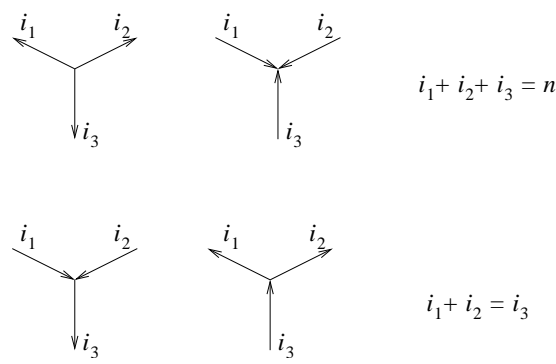

Fig. 61. The four types of vertices

To every such graph $\Gamma$ an invariant $\langle\Gamma\rangle$ is assigned, taking values in $\mathbb{Z}\left[q, q^{-1}\right]$. The invariant is unchanged if the orientation of an edge is reversed simultaneously with changing the label from $i$ to $n-i$. We use this transformation to reduce our considerations to graphs with all labels at most $n / 2$. We split the vertices into four types by the number (zero to three) of edges oriented into the vertex (see Figure 61).

The construction of Section 6 generalizes to a homology theory $H(\Gamma)$ for graphs $\Gamma$ as above. We will now sketch this generalization and its conjectural extension to link homology. For convenience, assume that $n$ is even.

Denote by $i(e)$ the number assigned to an edge $e$, and select a set $s(e)$ of cardinality $i(e)$ such that the sets assigned to different edges are disjoint. Suppose that a vertex $v$ bounds the edges $e_{1}, e_{2}, e_{3}$. To $v$ we assign the potential

$$
w_{v}=\sum_{j \in s\left(e_{1}\right) \sqcup s\left(e_{2}\right) \sqcup s\left(e_{3}\right)} \pm x_{j}^{n+1}
$$

where the sign is + if the edge $e_{k}$ leaves $v$ and $j \in s\left(e_{k}\right)$, and - otherwise (note that the potential of an edge $e$ is $\pm \sum_{j \in s(e)} x_{j}^{n+1}$ ).

Let $\mathbb{Q}[s(e)]$ be the ring of polynomials in $x_{j}, j \in s(e)$, and denote by $S(e)$ its subring of symmetric polynomials. Let

$$
R_{v}:=S\left(e_{1}\right) \otimes_{\mathbb{Q}} S\left(e_{2}\right) \otimes_{\mathbb{Q}} S\left(e_{3}\right)
$$

be the tensor product of the three rings. Then $w_{v} \in R_{v}$.

Consider first the case when the edges $e_{1}, e_{2}, e_{3}$ are oriented away from $v$. Then $i\left(e_{1}\right)+i\left(e_{2}\right)+i\left(e_{3}\right)=n$. Let $\sigma_{k}(v)$ be the $k$ th elementary symmetric function in the $x_{j}$ 's for $j$ in the set $s\left(e_{1}\right) \sqcup s\left(e_{2}\right) \sqcup s\left(e_{3}\right)$. Write

$$
w_{v}=\sum_{j} x_{j}^{n+1}=\sum_{k=1}^{n} \sigma_{k}(v) g_{k}(v)
$$

for some $g_{k}(v)$ 's (which are not uniquely defined, of course).

To $v$ we assign the factorization $C_{v}$ which is the tensor product of

$$
R_{v} \stackrel{g_{k}(v)}{\longrightarrow} R_{v} \stackrel{\sigma_{k}(v)}{\longrightarrow} R_{v}
$$

over $1 \leq k \leq n$. 
If the edges $e_{1}, e_{2}, e_{3}$ are all oriented towards $v$, then

$$
w_{v}=-\sum_{j} x_{j}^{n+1}=-\sum_{k=1}^{n} \sigma_{k}(v) g_{k}(v),
$$

and to $v$ we assign the factorization $C_{v}$ which is the tensor product of

$$
R_{v} \stackrel{g_{k}(v)}{\longrightarrow} R_{v} \stackrel{-\sigma_{k}(v)}{\longrightarrow} R_{v}
$$

over $1 \leq k \leq n$.

Suppose now that, say, $e_{1}, e_{2}$ are oriented away from $v$ and $e_{3}$ towards $v$. Then $i\left(e_{3}\right)=i\left(e_{1}\right)+i\left(e_{2}\right)$ and

$$
w_{v}=\sum_{j \in s\left(e_{1}\right) \sqcup s\left(e_{2}\right)} x_{j}^{n+1}-\sum_{j \in s\left(e_{3}\right)} x_{j}^{n+1} .
$$

Let $\sigma_{k}^{\prime}$ be the $k$ th elementary symmetric function in $x_{j}, j \in s\left(e_{1}\right) \sqcup s\left(e_{2}\right)$, and $\sigma_{k}^{\prime \prime}$ be the $k$ th elementary symmetric function in $x_{j}, j \in s\left(e_{3}\right)$. Since $w_{v}$ belongs to the ideal of $R_{v}$ generated by the differences $\sigma_{k}^{\prime}-\sigma_{k}^{\prime \prime}$, we can write

$$
w_{v}=\sum_{k=1}^{i\left(e_{3}\right)}\left(\sigma_{k}^{\prime}-\sigma_{k}^{\prime \prime}\right) g_{k}(v)
$$

for some $g_{k}(v) \in R_{v}, 1 \leq k \leq i\left(e_{3}\right)$. Now assign to the vertex $v$ the factorization $C_{v}$ which is the tensor product of

$$
R_{v} \stackrel{g_{k}(v)}{\longrightarrow} R_{v} \stackrel{\sigma_{k}^{\prime}-\sigma_{k}^{\prime \prime}}{\longrightarrow} R_{v}
$$

for $1 \leq k \leq i\left(e_{3}\right)$.

Likewise, assign to $v$ the tensor product of

$$
R_{v} \stackrel{g_{k}(v)}{\longrightarrow} R_{v} \stackrel{\sigma_{k}^{\prime \prime}-\sigma_{k}^{\prime}}{\longrightarrow} R_{v}
$$

if two edges $e_{1}, e_{2}$ are oriented inwards, and $e_{3}$ outwards.

If $\Gamma$ does not contain loops, define $H(\Gamma)$ as the homology of the 2complex $C(\Gamma)$ which is the tensor product of $C_{v}$ over all vertices $v$ of $\Gamma$. The tensor product is taken over intermediate rings $S(e)$ for various edges $e$, so that $C(\Gamma)$ is a finite-rank module over the ring $\bigotimes_{e} S(e)$.

When loops are present in $\Gamma$, a loop labeled $i$ will "contribute" to the tensor product $C_{v}$ the cohomology of the Grassmannian $\operatorname{Gr}(i, n)$ of $i$-dimensional subspaces in $\mathbb{C}^{n}$.

We conjecture that $H(\Gamma)$ has cohomology in only one out of its two degrees. An additional $\mathbb{Z}$-grading on $H(\Gamma)$ comes from grading on the rings $S(e)$, with $\operatorname{deg}\left(x_{j}\right)=2$. We conjecture that the graded dimension of $H(\Gamma)$ is the invariant $\langle\Gamma\rangle$ (up to obvious normalizations such as multiplication by a power of $q$, etc.).

The above construction should work when $n$ is even. If $n$ is odd, add a variable $x_{v}$ to each vertex with all edges oriented in, and to each vertex 
with all edges out. Then add $x_{v}^{2}$ to the potential $w_{v}$, enlarge the ring $R_{v}$ by adjoining the variable $x_{v}$, and modify the factorization $C_{v}$ correspondingly. We omit the details.

Given a diagram $D$ of an oriented framed link $L$ colored by numbers from 1 to $n-1$, each crossing can be resolved in a number of ways into planar graphs, and the invariant $P_{n}(L)$ is a linear combination of $\langle\Gamma\rangle$ for various resolutions $\Gamma$ of $D$, with coefficients which are plus or minus powers of $q$ (see Section 5 of $[\mathrm{MOY}])$. We conjecture that, likewise, the $\mathbb{Q}$-vector spaces $H(\Gamma)$ can be naturally strung together into a complex $C(D)$ whose (bigraded) cohomology groups are invariants of $L$ and have graded Euler characteristic $P_{n}(L)$. For each $n$, this homology theory of colored oriented framed links in $\mathbb{R}^{3}$ should be functorial under (oriented, framed, and suitably decorated) link cobordisms; it should also extend to a homology theory of (decorated) spatial trivalent graphs, and be functorial under (carefully defined) graph cobordisms in $\mathbb{R}^{3} \times[0,1]$.

\section{References}

[AGV] V. I. Arnold, S. M. Gusein-Zade and A. N. Varchenko, Singularities of Differentiable Maps, Vol. I, Monogr. Math. 82, Birkhäuser, Boston, 1985.

[BL] J. Baez and L. Langford, Higher-dimensional algebra IV: 2-tangles, Adv. Math. 180 (2003), 705-764; math.QA/9811139.

[BN1] D. Bar-Natan, On Khovanov's categorification of the Jones polynomial, Algebra Geom. Topol. 2 (2002), 337-370; math.QA/0201043.

[BN2] -, Khovanov's homology for tangles and cobordisms, Geom. Topol. 9 (2005), 1443-1499; math.GT/0410495.

[Be] D. J. Benson, Representations and Cohomology I. Basic Representation Theory of Finite Groups and Associative Algebras, Cambridge Stud. Adv. Math. 30, Cambridge Univ. Press, 1995.

[BV] B. Blok and A. N. Varchenko, Topological conformal field theories and the flat coordinates, Int. J. Modern Phys. A7 (1992), 1467-1490.

[B] R.-O. Buchweitz, Maximal Cohen-Macaulay modules and Tate cohomology over Gorenstein rings, preprint, circa 1986.

[BEH] R.-O. Buchweitz, D. Eisenbud, and J. Herzog, Cohen-Macaulay modules on quadrics (with an appendix by R.-O. Buchweitz), in: Lecture Notes in Math. 1273, Springer, 1987, 58-116.

[BGS] R.-O. Buchweitz, G.-M. Greuel and F.-O. Schreyer, Cohen-Macaulay modules on hypersurface singularities II, Invent. Math. 88 (1987), 165-182.

[CS1] J. S. Carter and M. Saito, Reidemeister moves for surface isotopies and their interpretation as moves to movies, J. Knot Theory Ramif. 2 (1993), 251-284.

[CS2] - - Knotted Surfaces and Their Diagrams, Math. Surveys Monogr. 55, Amer. Math. Soc., 1998.

[Di] A. Dimca, Topics on Real and Complex Singularities, Adv. Lectures in Math., Vieweg, 1987. 
[D] B. Dubrovin, Geometry and analytic theory of Frobenius manifolds, in: Proc. Int. Congress Math., Vol. II (Berlin, 1998), Doc. Math. 1998, Extra Vol. II, 315-326; math.AG/9807034.

[E1] D. Eisenbud, Homological algebra on a complete intersection, with an application to group representations, Trans. Amer. Math. Soc. 260 (1980), 35-64.

[E2] - Commutative Algebra, with a View Toward Algebraic Geometry, Grad. Texts in Math. 150, Springer, New York, 1995.

[EP] V. Ene and D. Popescu, Rank one maximal Cohen-Macaulay modules over singularities of type $Y_{1}^{3}+Y_{2}^{3}+Y_{3}^{3}+Y_{4}^{3}$, math. AC/0303151.

[F] J. E. Fischer, Jr., 2-categories and 2-knots, Duke Math. J. 75 (1994), 493526.

[FKS] I. B. Frenkel, M. Khovanov, and O. Schiffmann, Homological realization of Nakajima varieties and Weyl group actions, Compos. Math. 141 (2005), 1479-1503; math.QA/0311485.

[GH] P. Griffiths and J. Harris, Principles of Algebraic Geometry, Wiley, 1978.

[HOMFLY] P. Freyd, D. Yetter, J. Hoste, W. B. R. Lickorish, K. Millett and A. Ocneanu, A new polynomial invariant of knots and links, Bull. Amer. Math. Soc. (N.S.) 12 (1985), 239-246.

[HP] J. Herzog and D. Popescu, Thom-Sebastiani problems for maximal CohenMacaulay modules, Math. Ann. 309 (1997), 677-700.

[J] M. Jacobsson, An invariant of link cobordisms from Khovanov's homology theory, Algebr. Geom. Topol. 4 (2004), 1211-1251; math.GT/0206303.

[KL1] A. Kapustin and Y. Li, D-branes in Landau-Ginzburg models and algebraic geometry, J. High Energy Phys. 2003, no. 12, 005; hep-th/0210296.

[KL2] - - - Topological correlators in Landau-Ginzburg models with boundaries, Adv. Theor. Math. Phys. 7 (2004), 727-749; hep-th/0305136.

[KL3] -, -, D-branes in topological minimal models: the Landau-Ginzburg approach, J. High Energy Phys. 2004, no. 7, 045; hep-th/0306001.

[KS] L. H. Kauffman and H. Saleur, Free fermions and the Alexander-Conway polynomial, Comm. Math. Phys. 141 (1991), 293-327.

[Kh1] M. Khovanov, A categorification of the Jones polynomial, Duke Math. J. 101 (1999), 359-426; math.QA/9908171.

[Kh2] -, A functor-valued invariant of tangles, Algebr. Geom. Topol. 2 (2002), 665-741; math.QA/0103190.

[Kh3] -, sl(3) link homology I, ibid. 4 (2004), 1045-1081; math.QA/0304375.

[Kh4] -, An invariant of tangle cobordisms, Trans. Amer. Math. Soc. 358 (2006), 315-327; math.QA/0207264.

[Kn] H. Knörrer, Cohen-Macaulay modules on hypersurface singularities. I, Invent. Math. 88 (1987), 153-164.

[Ku] V. Kulikov, Mixed Hodge Structures and Singularities, Cambridge Univ. Press, 1998.

[M] E. Martinec, Algebraic geometry and effective Lagrangians, Phys. Lett. B 217 (1989), 431-437.

[MC] D. McDuff and D. Salamon, J-holomorphic Curves and Quantum Cohomology, Univ. Lecture Ser. 6, Amer. Math. Soc., 1994.

[MOY] H. Murakami, T. Ohtsuki and S. Yamada, HOMFLY polynomial via an invariant of colored plane graphs, Enseign. Math. (2) 44 (1998), 325-360.

[NN] T. Nakayama and C. Nesbitt, Note on symmetric algebras, Ann. of Math. 39 (1938), 659-668. 
[O] D. Orlov, Triangulated categories of singularities and D-branes in LandauGinzburg models, Proc. Steklov Inst. Math. 246 (2004), 227-248; math.AG/ 0302304.

[OS] P. Ozsváth and Z. Szabó, Holomorphic disks and knot invariants, Adv. Math. 186 (2004), 58-116; math.GT/0209056.

[P] D. Popescu, Cohen-Macaulay representation, in: Algebra-Representation Theory (Constanţa, 2000), NATO Sci. Ser. II Math. Phys. Chem. 28, Kluwer, Dordrecht, 2001, 249-256.

[PT] J. Przytycki and P. Traczyk, Conway algebras and skein equivalence of links, Proc. Amer. Math. Soc. 100 (1987), 744-748.

[Ra] J. Rasmussen, Floer homology and knot complements, PhD Thesis, Harvard Univ., 2003; math.GT/0306378.

[RT] N. Reshetikhin and V. Turaev, Ribbon graphs and their invariants derived from quantum groups, Comm. Math. Phys. 127 (1990), 1-26.

[R] L. Rozansky, Topological A-models on seamed Riemann surfaces, Theor. Math. Phys. 11 (2007), 517-529; hep-th/0305205.

[Sa] K. Saito, Period mapping associated to a primitive form, Publ. RIMS 19 (1983), 1231-1264.

[S] F.-O. Schreyer, Finite and countable CM-representation type, in: Singularities, Representation of Algebras, and Vector Bundles (Lambrecht, 1985), Lecture Notes in Math. 1273, Springer, Berlin, 1987, 9-34.

[Sh] A. Shumakovitch, http://www.geometrie.ch/KhoHo/.

[VW] C. Vafa and N. Warner, Catastrophes and the classification of conformal theories, Phys. Lett. B 218 (1989), 51-58.

[Y1] Y. Yoshino, Cohen-Macaulay Modules over Cohen-Macaulay Rings, London Math. Soc. Lecture Note Ser. 146, Cambridge Univ. Press, 1990.

[Y2] - Tensor products of matrix factorizations, Nagoya Math. J. 152 (1998), $39-56$.

Department of Mathematics

Columbia University

New York, NY 10025, U.S.A.

E-mail: khovanov@math.columbia.edu
Department of Mathematics

University of North Carolina

Chapel Hill, NC 27599, U.S.A.

E-mail: rozansky@math.unc.edu

Received 22 October 2007;

in revised form 2 February 2008 